Gillich et al., p. 1

\title{
Alveoli form directly by budding led by a single epithelial cell
}

\author{
Astrid Gillich ${ }^{1}$, Krystal R. St. Julien$^{1}$, Douglas G. Brownfield ${ }^{1}$, \\ Kyle J. Travaglini ${ }^{1}$, Ross J. Metzger ${ }^{2}$, and Mark A. Krasnow ${ }^{1 *}$ \\ ${ }^{1}$ Department of Biochemistry and Howard Hughes Medical Institute, \\ ${ }^{2}$ Department of Pediatrics, Division of Cardiology, \\ Stanford University School of Medicine, Stanford, California 94305, USA
}

*Corresponding author: krasnow@stanford.edu, 650-723-7191 (phone), 650-723-6783 (fax) 
Gillich et al., p. 2

\begin{abstract}
Oxygen passes along the ramifying branches of the lung's bronchial tree and enters the blood through millions of tiny, thin-walled gas exchange sacs called alveoli. Classical histological studies have suggested that alveoli arise late in development by a septation process that subdivides large air sacs into smaller compartments. Although a critical role has been proposed for contractile myofibroblasts, the mechanism of alveolar patterning and morphogenesis is not well understood. Here we present the three-dimensional cellular structure of alveoli, and show using single-cell labeling and deep imaging that an alveolus in the mouse lung is composed of just 2 epithelial cells and a total of a dozen cells of 7 different types, each with a remarkable, distinctive structure. By mapping alveolar development at cellular resolution at a specific position in the branch lineage, we find that alveoli form surprisingly early by direct budding of epithelial cells out from the airway stalk between enwrapping smooth muscle cells that rearrange into a ring of 3-5 myofibroblasts at the alveolar base. These alveolar entrance myofibroblasts are anatomically and developmentally distinct from myofibroblasts that form the thin fiber partitions of alveolar complexes ('partitioning' myofibroblasts). The nascent alveolar bud is led by a single alveolar type 2 (AT2) cell following selection from epithelial progenitors; a lateral inhibitory signal transduced by Notch ensures selection of only one cell so its trailing neighbor acquires AT1 fate and flattens into the cup-shaped wall of the alveolus. Our analysis suggests an elegant new model of alveolar patterning and formation that provides the foundation for understanding the cellular and molecular basis of alveolar diseases and regeneration.
\end{abstract}

\title{
One Sentence Summary
}

We report a direct budding mechanism of alveolar development distinct from the classical model of subdivision ('septation') of large air sacs. 
Gillich et al., p. 3

\section{Introduction}

The respiratory surface of the mammalian lung is formed by millions of regularly spaced, densely packed, thin-walled air sacs called alveoli arranged as single units or in small groups along the tubular walls or ends of airways $(1,2)$. Air passes along the airways into alveoli, where oxygen diffuses across the alveolar wall to reach circulating red blood cells, which distribute it throughout the body (3). Classic studies of lung structure revealed the geometry and architecture of respiratory airways $(1,2,4-8)$ and provided stereological estimates of alveolar number (2-4 million in mice), size (50 $\mu \mathrm{m}$ mean diameter), and surface area $\left(6 \times 10^{3} \mu \mathrm{m}^{2}\right)(9-12)$. However, the extreme thinness of alveolar cells and dense packing of alveoli make it difficult to count individual cells and define their structures and boundaries on histological sections. Electron microscopy and serial section reconstruction resolved the fine structure of the air-blood barrier and the organization of the alveolar epithelium (13-18), but the techniques are laborious and have not been used to systematically define the morphologies, numbers and arrangement of the different cell types in an alveolus. Hence the three-dimensional cellular structure of alveoli has not been resolved.

There is an urgent need to define the cellular structure and formation of alveoli since they are the sites of major, life-threatening lung diseases. These include chronic diseases such as emphysema/chronic obstructive pulmonary disease (COPD), idiopathic pulmonary fibrosis (IPF), and bronchopulmonary dysplasia (BPD), characterized by impaired or arrested alveolar development, as well as the acute respiratory distress syndromes accompanying severe injury or alveolar damage following infection, as in SARS, MERS, and the current Covid-19 pandemic (19-24). In these diseases enlargement, destruction, or flooding of alveoli, or changes in 
thickness or composition of their walls, result in a loss or lack of gas exchange surface, causing a decline in lung function or even respiratory failure.

The textbook model of alveolar development posits that the epithelium forms large sacs ('sacculation') that are later subdivided into alveoli by contractile myofibroblasts ('septation') (Fig. S1) (25-29). Alveolar septa are thought to arise postnatally in rodents $(25,27)$ and beginning at fetal stages in humans $(30,31)$, initially as shallow crests that then deepen, with myofibroblasts (first termed 'contractile interstitial cells' because of their characteristic ultrastructure and contractile elements similar to smooth muscle) and elastic fibers located at septal tips (32-35). Evidence to support the model is limited since developing alveoli have not been visualized at cellular resolution and in three dimensions, and the origin and role of myofibroblasts are not well defined. Although recent studies have begun to localize and characterize myofibroblasts and their progenitors (36-39), it remains unclear when and how myofibroblasts arise and are recruited to epithelial sacs, how they get positioned or anchored on the saccular walls, and how they migrate inward or contract to produce alveoli. Despite remarkable recent progress in alveolar development (40-45) including dynamic cell imaging (46) and elucidation of the complete gene expression program of the alveolar epithelium (47), our understanding of the mechanisms of alveolar patterning and morphogenesis and the coordination and interactions between cells remains rudimentary.

Here we use mosaic labeling, clonal analysis and deep imaging to elucidate the threedimensional cellular structure of alveoli in the mouse lung. We then analyze alveolar development at single-cell resolution and at a specific position in the branch lineage. We show that alveoli form much earlier and more simply than previously thought - by budding of the epithelium on airway stalks between smooth muscle cells that rearrange to become 
myofibroblasts at the alveolar openings. A single AT2 cell is selected to lead the alveolar outgrowth; a lateral inhibitory signal mediated by Notch prevents induction of AT2 fate in surrounding progenitors, which then develop as AT1 cells. Our analysis suggests an elegant new mechanism of alveolar patterning and development distinct from the classical septation model.

\section{The three-dimensional cellular structure of alveoli in the mouse lung}

To visualize the three-dimensional architecture of individual alveoli, we labeled the network of elastic fibers that surround and support the entrance to alveoli $(48,49)$ by incubating inflated and fixed lungs in fluorescent hydrazide (Fig. S2) (50). Deep imaging and threedimensional reconstruction revealed three classes of alveolar elastic fibers delineated by thickness and localization within or around alveoli (thick, thin and microvascular fibers; Fig. S2C). These classes allowed us to discern two types of alveoli: simple alveoli with the entrance formed by a ring of thick fibers (Fig. 1, A and B, Fig. S2D, and Movie S1), and groups of 2-8 alveoli (alveolar complexes) with a shared entrance encircled by thick fibers and partitions formed by thin fibers (Fig. 1, C and D, Fig. S2E, and Movie S2). This is consistent with previous descriptions of the geometry of respiratory airways $(1,2,8,51)$. Single alveolar units and complexes are intermingled throughout respiratory airways, although simple outpocketings are especially obvious and may be more abundant on early generations (Fig. S2, F and G).

To elucidate the cellular structure of alveoli, we determined the morphologies of alveolar cells and their arrangement by labeling single cells using genetic strategies and analyzing their position within alveoli relative to the ring of elastic fibers at the alveolar entrance. Tamoxifeninducible CreER lines (Table S1), in which Cre recombinase is fused to a modified estrogen receptor to control the timing and extent of labeling, were crossed to a Cre reporter in which recombination of a loxP flanked stop cassette leads to expression of a fluorescent protein. 
Limiting doses of tamoxifen were used to induce rare recombination events (100-800 events per lobe) that labeled isolated cells. Three-dimensional reconstruction of single cells and the surrounding fibers revealed that alveolar cells have a stunning variety of shapes and unusual features and arrangements (Fig. 1, E to K).

There is on average just a single alveolar type 1 (AT1) cell per alveolus (Fig. 1E, Fig. S3, and Fig. S5, A, B and G). It is typically a cup-shaped cell with its thin, expansive cytoplasmic extensions covering nearly the entire alveolar surface $\left(5,500 \mu \mathrm{m}^{2}\right)(12)$. However, the arrangement of AT1 cells in alveoli is variable and individual cells can span 2-5 alveoli (Fig. S3 and S4), consistent with descriptions of 'non-nucleated plates' (17). The majority (96\%, n=100) of AT1 cells have one or more (up to 9) holes ('pores of Kohn' (52)) of circular or oval shape and varying sizes (Fig. S5B). AT1 cells form junctions with each other and with neighboring AT2 cells that are strategically positioned between alveoli as single cuboidal or elongated cells (Fig. 1F and Fig. S5C) (17). Although there is typically also just a single AT2 cell per alveolus (Fig. S5G), individual AT2 cells often have multiple apical surfaces contributing to 2-4 alveoli ( $80 \%$ of AT2 cells with $>1$ apical domain, $n=500$ ).

The alveolus is surrounded by a dense mesh of capillaries made up of two distinct, intermingled cell types with 'swiss cheese' morphologies (Fig. 1G and H, and Fig. S6, A and B) $(53,54)$. Pericytes and alveolar fibroblasts are complex, branched cells with long $(>50 \mu \mathrm{m})$, thin processes spanning multiple alveoli (Fig. 1, I and J, Fig. S5, E and F, and Fig. S6, C and D). Myofibroblasts, by contrast, are simple, spindle-shaped cells with 2 or 3 cell processes that are aligned with thick elastic fibers at the alveolar entrance (Fig. 1K and Fig. S5D). Three to five myofibroblasts form a ring around the alveolar entrance (Fig. S5G), with their cell bodies located 
at the intersections of neighboring alveoli and with the number of cells per ring corresponding to the number of intersections.

These clonal labeling experiments combined with immunostaining against common antigens, deep imaging, 3D reconstruction and quantification (Figs. S3-S6 and Methods) allowed us to define the three-dimensional cellular structure of an alveolus (Fig. 1L and Fig. S5G). A simple alveolus is composed of just 2 epithelial cells, a single AT2 cell and an AT1 cell that covers the majority of the alveolar surface, 3-4 capillary endothelial cells (of 2 cell types: aerocytes and general capillary cells; relative abundance 1:4), and 5-9 stromal cells including 1-2 pericytes, 1-2 fibroblasts, and 3-5 myofibroblasts. Thus, an alveolus in the mouse lung is composed of just 10-15 cells of 7 major types, excluding immune cells. This is $2-3$ fold fewer cells than previous estimates based on stereology of thin sections of alveoli (55), which apparently did not distinguish complexes from individual alveoli. Alveolar complexes contain proportionally more cells than simple alveoli, scaled to the number of alveolar units (“chambers") in the complex (Fig. S4).

\section{Mapping alveolar development at cellular resolution at a defined position in the lung}

To elucidate the cellular mechanisms that initiate and control alveolar patterning, we analyzed the earliest events in alveolar development by mapping the process at a specific position in the branch lineage (56). In the left primary bronchus (L) lineage, the third anterior branch (A3) forms by domain branching on the anterior aspect of the first secondary branch (L1) and generates 4-6 generations of daughter branches by planar and orthogonal bifurcations (Fig. 2A). By immunostaining of whole lobes for Sox 2 (or incubation with fluorescent streptavidin to detect biotin expressed by epithelial cells), which allows visualization of conducting airways (57, 58), we mapped the boundary between conducting and respiratory airways on L.L1.A3 and 
analyzed when the boundary forms during development (Fig. S7). We found that the boundary is positioned on L.L1.A3 daughters at or just distal to the bifurcation between airway generations 4 and 5 (Fig. S7, A to C). Consistent with previous studies (57), the boundary is set by embryonic day (E) 16.5 (Fig. 2, B to D, and Fig. S7D), and branches distal to the boundary form respiratory airways.

Immunostaining of whole lobes for epithelial and smooth muscle markers showed that at E16.5 smooth muscle actin (SMA)-positive cells surround epithelial tubes destined to form respiratory airways (Fig. 2C). The cells have an elongated morphology and circumferential orientation typical of smooth muscle on conducting airways (Fig. 2E). Just one day later (E17.5), the smooth muscle pattern is less organized (Fig. 2D), with the cells oriented along airway stalks at various angles (Fig. 2F). Already at this stage, the cells are aligned with elastic fibers (Fig. S8). This suggested that smooth muscle cells on embryonic airways rearrange and become myofibroblasts at the alveolar entrance.

\section{Airway smooth muscle cells become myofibroblasts at the alveolar entrance}

To test whether airway smooth muscle is a source of myofibroblasts, we used a genetic strategy to specifically label and trace smooth muscle on embryonic airways (59). We combined the airway smooth muscle-specific Lgr6-EGFP-IRES-CreERT2 (Lgr6-CreER) (60) knock-in allele with the Cre reporter Rosa26-tdTomato (61) and induced recombination at E14.5 with a saturating dose of tamoxifen (Fig. 3A and Fig. S9). Analysis of the labeling on postnatal day 10 (P10) showed lineage-labeled cells on first and second order respiratory airways (Fig. 3B). The cells express SMA and are aligned with thick elastic fibers at alveolar openings (Fig. 3B'), demonstrating that airway smooth muscle cells become entrance myofibroblasts. Alveolar entrance myofibroblasts express Eln (tropoelastin) as shown by single-cell RNA-sequencing 
(scRNAseq) and single-molecule in situ hybridization (smFISH) (Fig. S10, F and G) (62, 63), confirming that the cells are indeed a source of elastin, a major component of elastic fibers.

To determine if myofibroblasts on later-generation branches also arise from the Lgr6 lineage, we induced recombination at a later embryonic timepoint by dosing E17.5 Lgr6-CreER; Rosa26-tdTomato lungs with tamoxifen (Fig. S10A). Lineage labeled cells were located on 3-4 generations of respiratory airways (Fig. S10, B and B'). Postnatal labeling (Fig. S10C) included distal respiratory airways (Fig. S10, D and D'). This shows that alveolar entrance myofibroblasts are derived from the Lgr6 lineage (Fig. S10H) on early and late generation airways.

To probe the potential and behavior of individual airway smooth muscle cells, we sparsely labeled smooth muscle cells by induction of rare recombination events in smooth muscle myosin heavy chain (SMMHC)-CreER; Rosa26-Rainbow lungs $(64,65)$ using limiting doses of tamoxifen (Fig. 3D). Airway clones induced at E15 and analyzed on postnatal day 10 were composed of 1 to 17 cells. Clones fell into 3 groups based on the location of daughter cells (Table S2). In the first group, cells of a clone were located exclusively around stalks of conducting airways ("airway smooth muscle clones", n=30 clones; 44\%; Fig. S11). In some of these clones $(37 \%)$ the daughter cells remained in close contact with their siblings, whereas in other clones (37\%) cells were dispersed, spanning 100-500 $\mu \mathrm{m}$ along the airway axis, implying extensive movement of daughter cells, as has been observed early in development (59). The second group was formed by clones spanning the boundary between conducting and respiratory airways ("mixed/boundary clones", n=8 clones; 12\%; Fig. 3E and Fig. S12). These clones were composed of both elongated cells with circumferential orientation around airway stalks (airway smooth muscle) and spindle-shaped cells aligned with elastic fibers at the alveolar entrance (myofibroblasts). This demonstrates that individual cells can give rise to both, establishing a 
lineage relationship between airway smooth muscle cells and myofibroblasts. The third group of clones was composed of cells located exclusively on respiratory airways ("myofibroblast clones", n=30 clones; 44\%; Fig. 3F and Fig. S13). Nearly all (98\%) labeled cells ( $\mathrm{n}=100$ cells scored from 30 clones) were aligned with alveolar elastic fiber rings. Although the majority (70\%) of myofibroblast clones were composed of more than one cell (Table S2), individual entrance rings were generally incompletely labeled, excluding a model of entrance ring formation by clonal proliferation. The differences in cell behavior our analysis uncovered, with only a fraction of labeled cells turning into myofibroblasts, may reflect differences in the potential of individual cells, or perhaps more likely be dictated by the environment (e.g., only daughter cells located beyond the boundary turn into myofibroblasts).

\section{Anatomical and developmental diversity of alveolar myofibroblasts}

Immunostaining and smFISH in postnatal (P10) lungs revealed two distinct types of myofibroblasts. Both types express SMA and the myofibroblast marker $F g f 18(66)$, but one population associates with thick elastic fibers that encircle the openings to simple alveoli and alveolar complexes (“entrance myofibroblasts”; Fig. 4, A, A', B, B' and C). Our embryonic and postnatal pulse-chase experiments with Lgr6-CreER; Rosa26-tdTomato showed that entrance myofibroblasts derive from the Lgr6 lineage (Fig. 3B' and Fig. S10, A to D) and persist in the adult lung (Fig. 3C and Fig. S10E). Although nearly all (98\%) of these cells turn off SMA, they remain aligned with thick elastic fibers and retain a simple morphology (Fig. 3C').

The second population also expresses SMA and the myofibroblast marker $F g f 18$, but these cells are aligned with thin elastic fibers that subdivide alveolar complexes ("partitioning myofibroblasts", Fig. 4, A, A', B, B' and C). Partitioning myofibroblasts also have a spindle shape, and their cell processes are anchored on the thick elastic fibers and entrance 
myofibroblasts at the openings to alveolar complexes. Unlike alveolar entrance myofibroblasts, partitioning myofibroblasts are not lineage labeled by Lgr6-CreER (Fig. 3B' and Fig. S10, A to D), hence they arise from another source.

These results suggest that there are two types of myofibroblasts with distinct anatomical locations and developmental origins: SMA+Fgf18+ Lgr6 lineage-derived entrance myofibroblasts that form the thick fiber rings at the base of simple alveoli and alveolar complexes, and SMA + Fgf18+ Lgr6 lineage-negative partitioning myofibroblasts that subdivide alveolar complexes. Simple alveoli have entrance but not partitioning myofibroblasts (Fig. 4C).

\section{Alveoli form by epithelial budding led by a single AT2 cell}

The alignment of SMA+ cells with thick elastic fiber rings on stalks of embryonic airways at E17.5 (Fig. S8) suggested that alveoli form much earlier than previously assumed (P4 in mice) (25) and by a mechanism distinct from septation.

To determine how alveoli arise on airways, we analyzed the epithelial pattern on stalks of L.L1.A3 daughter branches by staining embryonic (E17.5) lobes for E-cadherin, and also for SMA and elastin (detected by hydrazide) to visualize the arrangement of epithelial cells relative to smooth muscle and elastic fibers. Surprisingly, we found small groups of 1-3 cuboidal epithelial cells protruding between smooth muscle cells on airway stalks (Fig. 5A and Fig. S14). The budding single cuboidal cells were surrounded by rings of 3 or more smooth muscle cells aligned with elastic fibers (Fig. 5, A' and A'"), indicating that these are the nascent entrance myofibroblasts organizing around budding epithelial cells and depositing elastin, supporting a model of alveolar development by direct budding.

Immunostaining for alveolar epithelial markers, including FGF receptor 2 (Fgfr2), showed that the single cuboidal cells are nascent AT2 cells (Fig. 5B and Fig. S15; see also 
Brownfield et al., in review (67)). Each budding AT2 cell is surrounded by 2-4 nascent AT1 cells that have begun to flatten (Fig. 5C). This suggests that the alveolar buds are led by single AT2 cells through the nascent entrance myofibroblasts that begin to synthesize elastic fibers and demarcate the alveolar entrance as the AT2 cell buds (Fig. 5D and E).

\section{A lateral inhibitory signal mediated by Notch ensures selection of a single AT2 cell}

The regular salt-and-pepper pattern of the alveolar epithelium with single AT2 cells surrounded by 2-4 nascent AT1 cells implies that the alveolar pre-pattern and spacing is established surprisingly early in the process, at or before E17.5. The salt-and-pepper pattern also raised the possibility that the balance between the alternative epithelial fates could be controlled by a Notch-mediated lateral inhibition mechanism (68). Indeed, an increase in AT2 cells has been observed in embryonic lungs mutant for Lunatic Fringe, a glycosyltransferase that facilitates Notch activation (69), and Notch signaling is active during the AT2-to-AT1 cell transition in culture (70). To determine if the Notch pathway is active in developing alveolar epithelial cells, we examined expression of the major transcriptional effector of Notch signaling, CBF1 (RBP-J), in embryonic (E16.5-18.5) lungs using a CBF1-H2B-Venus reporter (71). Immunostaining showed nuclear Venus localization in nascent AT1 cells (and endothelial plexus) but little or none in alveolar epithelial progenitors or budding AT2 cells marked by Fgfr2 (Fig. 6A and Fig. S16, A and B). Immunostaining for the Notch target Hes1 also showed pathway induction in nascent AT1 cells (Fig. S16, C and D). Thus, the Notch pathway is activated in the AT1 lineage during alveolar development.

To test if Notch signaling plays a role in alveolar patterning, we inhibited the pathway in developing (E16-E18.5) lungs using the $\gamma$-secretase inhibitor DBZ, which prevents ligandinduced proteolytic processing and activation of Notch (72). Whereas the majority (88 $\pm 5 \%)$ of 
AT2 cells in control lungs ( $\mathrm{n}=500$ AT2 cells scored in 6 animals) were found as single (isolated) AT2 cells at E18.5, only rare AT2 cells $(11 \pm 4 \%)$ were selected as single cells in DBZ-treated lungs ( $n=500$ cells in 6 animals), with the rest $(89 \pm 4 \%)$ found in clusters (Fig. 6, B to D). To determine if Notch inhibition enhances AT2 cell proliferation, as observed at postnatal stages (73), we performed immunostaining for Ki67. AT2 cell proliferation was comparable in DBZtreated and control lungs $(1.2 \pm 0.6 \% \mathrm{Ki} 67+\mathrm{AT} 2$ cells in DBZ-treated lungs; $1.5 \pm 0.5 \%$ in controls; Fig. 6E and Fig. S16E). This suggests that Notch signaling is required for alveolar patterning and specifically for selection of single AT2 cells, rather than to regulate their proliferation. Technical, developmental timing or background differences could explain the discrepancy between our findings and a previous study of Notch function (73).

To identify the relevant receptor and ligand, we analyzed expression of Notch pathway components in scRNAseq data for developing mouse lung $(47,74)$. This confirmed Notch pathway activation (assessed by Hes 1 expression) in the AT1 lineage. However, it also revealed potential signaling complexity. Expression of multiple receptors (Notch1-4) and ligands (Jagl, Jag2, Dll1, Dll4) was detected in the developing alveolar epithelium (Fig. S17, A and B) along with non-canonical ligands $D l k 1$ and $D l k 2$, with $D l k 1$ induced in the AT2 lineage. Other (nonepithelial) alveolar cells also expressed ligand genes (Fig. S17, B and C) identifying other possible sources of signal.

To test for Notch-mediated lateral inhibition, we used a cell culture system in which alveolar epithelial progenitors from E16.5 lungs are purified away from other alveolar cells and cultured alone in Matrigel; addition of Fgf7 or Fgf10 to the cultures induces formation of alveolus-like epithelial spheroids ('alveolospheres') comprised of intermingled AT1 and AT2 cells (see Brownfield et al. (67)) (Fig. 6, F and G). Inhibition of Notch signaling by addition of 
the $\gamma$-secretase inhibitor DAPT to the cultures increased the percentage of cells selected as AT2 cells from $58 \pm 4 \%$ in controls to $87 \pm 3 \%$ ( $\mathrm{n}=500$ cells scored in 3 experiments). Similarly, blocking antibodies against Notch1 and Notch2 (75) increased AT2 cell selection to $72 \pm 7 \%$, and $88 \pm 5 \%$, respectively. These results support a role for Notch signaling mediated by Notch1 and Notch2 in alveolar epithelial patterning and lateral inhibition of AT2 fate (Fig. 6H). However, the culture experiments do not exclude the possibility that other (non-epithelial) sources could also contribute to alveolar epithelial patterning in vivo. 


\section{Discussion}

Our analysis of alveolar patterning and morphogenesis reveals a new mechanism by which alveoli form in the lung. In contrast to the classical ('septation') model in which the epithelium gives rise to large sacs that are later subdivided into alveoli by myofibroblasts, we show that alveoli form early and directly by budding and outgrowth of the epithelium from airway stalks between enwrapping smooth muscle cells (Fig. 7). These smooth muscle cells rearrange to become a ring of myofibroblasts at the alveolar entrance. This surprisingly simple and elegant way to form alveoli relies on precise spatial and temporal control of epithelial patterning and coordination with surrounding cells.

We propose that the initiating event in alveolar development is the selection of a single AT2 cell that first becomes apparent by Fgfr2 restriction in the embryonic lung (see also Brownfield et al., in review (67)) right after the boundary is set between conducting and respiratory airways (57). The nascent AT2 cell leads the alveolar outgrowth and is the source of one or more signals to surrounding cells: a lateral inhibition signal to neighboring epithelial cells mediated by Notch1 and Notch2 that prevents selection of more than one AT2 cell, and possibly a signal to nearby smooth muscle to rearrange to form entrance myofibroblasts and synthesize elastin. Our data support a mechanism of alveolar patterning analogous to Drosophila tracheal branching morphogenesis (76) where a single cell is selected by Fgf signaling to take the lead position in budding, and Notch signaling prevents selection of additional cells.

While Fgf and Notch signaling together control the selection and spacing of leading AT2 cells, and hence set the alveolar pattern, at least one other signal to the epithelium appears necessary to confer AT1 fate on surrounding cells and generate alveoli because expression of a constitutively-active Notch protein was insufficient to induce AT1 differentiation in cultured 
alveolar epithelial progenitors (not shown). A mechanical signal may play a role in AT1 specification and initial flattening, as suggested by a recent study (46), and pressure-induced forces may ensure further flattening and postnatal outgrowth of alveoli. Whatever the nature of this signal, it can be provided in cultures of purified alveolar epithelial cells induced with Fgf because such cultures readily form alveolospheres with intermingled AT1 and AT2 cells (Fig. 6F; see also Brownfield et al., in review (67)).

In our model of alveolar development, airway smooth muscle is the long sought source of entrance myofibroblasts. They synthesize elastin and are aligned with thick elastic fibers at the base of simple alveoli and alveolar complexes. Although the cells turn off smooth muscle actin in the postnatal (P12-15) lung, some and possibly all of them continue to be associated with alveolar openings - they may get anchored to the fibers or adhere to the basement membrane of AT1 or capillary cells through specialized junctional complexes (34). Genetic tools to specifically label and manipulate entrance myofibroblasts are needed to directly test the requirement of the cells in alveolus formation and to elucidate their role in the mature lung. Identification of the signals that recruit or activate them to make or repair fibers may suggest ways to prevent or reverse alveolar elastin destruction in age-associated and heritable forms of emphysema, which could lead to new strategies to replace damaged or missing alveolar entrance rings and inform treatments.

Our results also identified a second type of alveolar myofibroblast with a distinct anatomical location and developmental origin: 'partitioning' myofibroblasts that subdivide alveolar complexes. Interestingly, two molecular types of alveolar myofibroblasts were recently reported in a preprint investigating the diversity of myofibroblasts by scRNAseq at different stages of embryonic and postnatal lung development (77). One population expresses Lgr6, Hhip 
and $C d h 4$ and persists post-alveologenesis, whereas the other expresses Pdgfra and undergoes developmental apoptosis. The Lgr6-expressing subset may correspond to entrance myofibroblasts that we show arise from the Lgr6 lineage, form the thick elastic fiber rings at the openings of simple and complex alveoli, and persist in the adult lung. The second population may be partitioning myofibroblasts that form thin fiber subdivisions of alveolar complexes.

The timing of alveolar complex formation and recruitment of partitioning myofibroblasts appears to coincide with the classical 'alveolarization' stage in the postnatal mouse lung (P4P30) $(25,27)$. This suggests that these myofibroblasts and the associated thin fiber partitions form after the entrance rings of simple and complex alveoli and by a distinct mechanism, presumably part of septation. Although we limited our in depth analysis of alveolar development to stalks of early forming airways to avoid caveats associated with inflation of air-filled lungs and variability in late forming branches, it will be important to find ways around these biological and technical issues to probe at cellular resolution the origin and dynamics of partitioning myofibroblasts and the steps beyond entrance ring formation that expand and subdivide some alveoli into complexes ("septation”). Such approaches could also elucidate other steps in alveolar growth and maturation, including interlinking of alveoli through transseptal AT2 cells with multiple apical sides (Fig. S5C) and specialized circular junctions between AT1 cells from adjacent airways to form pores of Kohn (Fig. S5B).

Our model of alveolar development by direct budding is supported by the threedimensional cellular structure of alveoli we elucidated by single-cell labeling. Remarkably, a simple alveolus in the mouse lung is composed of just 10-15 cells of 7 major cell types (excluding immune cells) including a single ('lead') AT2 cell, a single AT1 cell and 3-5 myofibroblasts at the entrance, which is 2-3 fold less than estimates from counting cells and 
alveoli on sections (55). Human alveoli are larger (200-300 $\mu$ m mean diameter compared to 50 $\mu \mathrm{m}$ in mouse) and are thought to contain at least ten times more cells $(12,16,55,78)$. Single-cell labeling approaches should now be developed to elucidate the precise number and structure of cells in human alveoli and to determine if the mechanism of alveolar development we uncovered in mice, with selection of single lead AT2 cells as the key initiating and patterning event followed by budding and recruitment of entrance myofibroblasts from smooth muscle, is conserved between species. Based on our analysis, the timing of initiation of alveolar development in mouse and humans may be more similar than previously thought. And because humans also appear to have both simple alveoli and complexes (2), the two types of alveolar myofibroblasts, with their distinct origins, anatomy, and functions, may also be conserved. Our analysis of the structure and development of alveoli provides a basis for such evolutionary comparisons and for investigations of how the structure and cells of alveoli are altered with age and in human diseases that compromise gas exchange, including chronic lung diseases that arise in infants born too early with incompletely developed alveoli (79). By identifying the cells and molecules that control alveolar patterning and morphogenesis, our analysis suggests ways to stimulate failed or arrested alveolar development in premature lungs, and may inform strategies to rebuild alveoli in injured, aged or diseased lungs. 
Gillich et al., p. 19

\section{Methods}

\section{Mice}

The following mouse strains were used: C57BL/6 (C57BL/6NCrl, Charles River Laboratories, strain 027) was the wild-type strain. Hopx-CreER (Hopx $x^{\text {tm2.1(cre/ERT2)Joe } / J) ~(80) ~(T h e ~ J a c k s o n ~}$ Laboratory, strain 017606), Foxa2-CreER (Foxa2 $\left.2^{\left.\text {tm2.1(cre/Esr } 1^{*}\right) M o o n} / \mathrm{J}\right)(81)($ The Jackson Laboratory, strain 008464), Shh-GFP-Cre (B6.Cg-Shh ${ }^{t m l(E G F P / c r e) C j t / J)}$ (82) (The Jackson Laboratory, strain 005622), VE-cadherin-CreER (Tg(Cdh5-cre/ERT2)CIVE23Mlia) (83) (provided by M. L. Iruela-Arispe), Apelin-CreER $\left(\operatorname{Apln}^{\text {tm1.1(cre/ERT2)Bzsh }}\right)(84)$ (provided by B. Zhou), Aplnr-CreER (Tg(Aplnr-cre/ERT2)\#Krh) (85) (provided by K. Red-Horse), PdgfraCreER (B6N.Cg-Tg(Pdgfra-cre/ERT)467Dbe/J) (86) (The Jackson Laboratory, strain 018280), Pdgfra-CreER (B6.129S-Pdgfra ${ }^{\text {tm1.1(cre/ERT2)Blh } / J) ~(87) ~(T h e ~ J a c k s o n ~ L a b o r a t o r y, ~ s t r a i n ~ 032770), ~}$

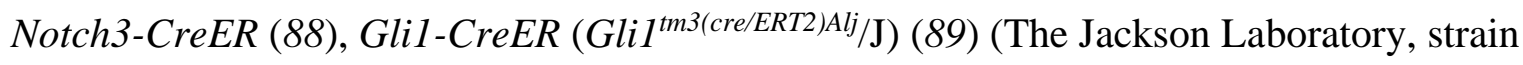

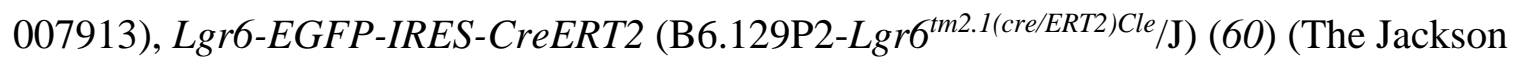
Laboratory, strain 016934), SMA-CreER (Tg(Acta2-cre/ERT2)12Pcn) (90), and SMMHC-CreER (B6.FVB-Tg(Myh11-cre/ERT2)1Soff/J) (64) (The Jackson Laboratory, strain 019079) were used for expression of Cre recombinase. Rosa26-mTmG (B6.129(Cg)-Gt(ROSA)26Sor ${ }^{\text {tm4(ACTB-tdTomato,- }}$ EGFP)Luo/J) (91) (The Jackson Laboratory, strain 007676), which expresses membrane targeted GFP after recombination, Rosa26-tdTomato (Gt(ROSA)26Sor $\left.{ }^{\text {tml4(CAG-tdTomato)Hze }}\right)$ (61) (The Jackson Laboratory, strain 007914), which expresses cytoplasmic tdTomato after recombination,

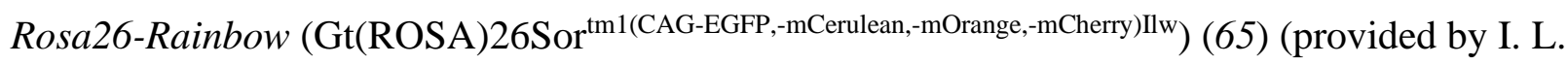
Weissman), which expresses cytoplasmic Cerulean, mOrange or mCherry after recombination,

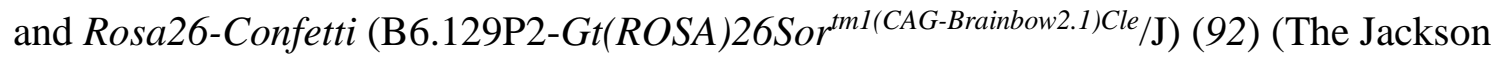
Laboratory, strain 017492), which expresses membrane targeted Cerulean CFP, nuclear GFP, 
cytoplasmic EYFP or cytoplasmic RFP after recombination, were used as Cre reporters. CBF1H2B-Venus (Tg(Cp-HIST1H2BB/Venus)47Hadj/J) (71) (The Jackson Laboratory, strain 020942), which expresses nuclear Venus under the control of CBF1 binding sites, was used to visualize Notch activity in developing lungs. All experimental mice and embryos were heterozygous (or hemizygous) for indicated alleles. Only female mice and embryos were used for experiments with Apelin-CreER, as Apelin is X-linked (84). Noon of the day a vaginal plug was detected was considered as E0.5. The day a litter was born was considered as P0. For induction of Cre recombinase activity, tamoxifen (Sigma, Cat. No. T5648) was dissolved in corn oil and administered by intraperitoneal (i.p.) injection unless otherwise noted. Animal experiments were performed in accordance with approved Animal Care and Use Committee protocols.

\section{Immunostaining}

Embryonic mouse lungs were dissected in PBS and fixed in 4\% paraformaldehyde (PFA) for 1 hour (E16.5), 1.5 hours (E17.5) or 2 hours $(\mathrm{E} 18.5)$ at $4^{\circ} \mathrm{C}$. Fixed lungs were washed three times in PBS for 15 minutes at $4^{\circ} \mathrm{C}$, then dehydrated through a PBS and methanol series into $100 \%$ methanol and stored at $-20^{\circ} \mathrm{C}$. For Sox 2 antibody stains, embryonic lungs were fixed in $0.5 \%$ PFA for 3 hours at room temperature, followed by washes and dehydration into methanol. For Fgfr2 stains, lungs were incubated in Dent's fixative (80\% methanol, 20\% dimethylsulfoxide (DMSO)) overnight at $4^{\circ} \mathrm{C}$, washed twice in $100 \%$ methanol and stored as above.

Postnatal mouse lungs were perfused with PBS, inflated with $2 \%$ low-melting point agarose (Thermo Fisher, Cat. No. 16520100) dissolved in PBS and fixed by immersion in 4\% PFA for 2 hours (P10) or 3-5 hours (adult) at $4{ }^{\circ} \mathrm{C}$ (or in $0.5 \%$ PFA for 5 hours at room temperature for Sox 2 antibody stains). Fixed tissue was washed three times in PBS for 30 
minutes and dehydrated in methanol as above. Lungs were rehydrated on the day of staining through a methanol and PBS series into PBS. Sections $(300-500 \mu \mathrm{m})$ were cut from left or right cranial lobes on a vibratome (Leica Biosystems). Rehydrated whole lobes or vibratome sections were blocked in $5 \%$ heat inactivated goat serum (or donkey serum for primaries raised in goat) in PBS with $0.5 \%$ Triton X-100 and 3\% BSA for 2 hours at room temperature and incubated in primary antibodies diluted in block for 3 nights at $4{ }^{\circ} \mathrm{C}$.

Primary antibodies used, at indicated concentrations, were: rat anti-E-cadherin (1:500, Thermo Fisher, Cat. No. 13-1900, clone ECCD-2), goat anti-Sox2 (1:150, Santa Cruz, Cat. No. sc-17320), rabbit anti-Fgfr2 (1:150, Santa Cruz, Cat. No. sc-122, C-17), Armenian hamster antiMuc1 (1:500, Thermo Fisher, Cat. No. HM1630P0, clone MH1), rabbit anti-SftpC (1:200, Millipore, Cat. No. AB3786), Syrian hamster anti-podoplanin (Pdpn; 1:50, DHSB, Cat. No. 8.1.1), rat anti-RAGE (1:500, R\&D, Cat. No. MAB1179, clone 175410), Armenian hamster antiPecam1 (1:300, Bio-Rad, Cat. No. MCA1370Z, clone 2H8), rabbit anti-NG2 (1:200, Millipore, Cat. No. AB5320), rat anti-Pdgfrb (1:100, eBioscience, Cat. No. 14-1402), rabbit anti-elastin (1:500, kindly provided by Robert Mecham), goat anti-Itga8 (integrin $\alpha)(1: 500$, R\&D, Cat. No. AF4076), goat anti-Pdgfra (1:200, R\&D Systems, Cat. No. AF1062), rabbit anti-RFP (1:300, Rockland, Cat. No. 600-401-379), chicken anti-GFP (1:500, Abcam, Cat. No. ab13970), and mouse anti-SMA conjugated to FITC (1:200, Sigma, Cat. No. F3777, clone 1A4), Cy3 (1:200, Sigma, Cat. No. C6198, clone 1A4), or Cy5 (1:200). The SMA-Cy5 conjugate was prepared by mixing $100 \mu \mathrm{g}$ of SMA antibody (Sigma, Cat. No. A2547, clone 1A4) with $4 \mu 1$ of Cy5 NHS Ester (GE Healthcare; $1 \mathrm{mg}$ dye dissolved in $10 \mu \mathrm{l}$ of anhydrous DMSO) and incubation for 2 hours at room temperature in the dark. Unbound dye was removed using a gel filtration column (P-30, Bio-Rad) equilibrated in PBS with $0.09 \%$ sodium azide. 
Following primary incubation, samples were washed in block six times for 1 hour and incubated in secondary antibodies conjugated to fluorescent dyes diluted 1:250 in block with added 4',6-diamidino-2-phenylindole (DAPI; 1:500 dilution of 1 mg/ml stock, Thermo Fisher) for 2 nights at $4^{\circ} \mathrm{C}$.

Secondary antibodies used, at indicated concentrations, were: goat anti-chicken Alexa Fluor 488 (Thermo Fisher, Cat. No. A-11039), goat anti-rabbit Alexa Fluor 568 (Thermo Fisher, Cat. No. A-11036), goat anti-rabbit Alexa Fluor 633 (Thermo Fisher, Cat. No. A-21070), goat anti-Armenian hamster Alexa Fluor 647 (Jackson Immunoresearch, Cat. No. 127-605-160), donkey anti-goat Alexa Fluor 488 (Thermo Fisher, Cat. No. A-11055), or donkey anti-rat Alexa Fluor 647 (Jackson Immunoresearch, Cat. No. 712-605-153).

Following secondary incubation, samples were washed in PBS with $0.1 \%$ Tween-20 six times for 1 hour, post-fixed in $4 \%$ PFA for 1 hour at $4^{\circ} \mathrm{C}$, washed again three times for 15 minutes in PBS and dehydrated into methanol as above. Samples were cleared in benzyl alcohol:benzyl benzoate (BABB; 1:2) for confocal imaging.

For signal amplification of rat anti-GFP primaries, inflated and fixed whole lobes or vibratome sections were bleached with 5\% hydrogen peroxide in methanol for 5 hours at room temperature, blocked as above and incubated in primary (rat anti-GFP, 1:200, ChromoTek, Cat. No. 3h9-100, clone 3H9) diluted in block as above. Samples were washed in block six times for 1 hour and incubated with horseradish peroxidase (HRP)-conjugated goat anti-rat IgG (Vector, Cat. No. PI-9401) diluted 1:150 in block for 2 nights at $4^{\circ}$ C. Samples were washed again in PBS with $0.1 \%$ Tween-20 six times for 1 hour and incubated in fluorescein tyramide reagent (Perkin Elmer, Cat. No. SAT701001EA) diluted 1:70 in amplification diluent for 50 minutes at room 
temperature. Samples were washed in PBS four times for 15 minutes, post-fixed and cleared as above.

For visualizing elastic fibers, lungs were stained with fluorescent hydrazide (50). Whole embryonic or postnatal lobes, dissected and fixed as above in 4\% PFA, were incubated with Alexa Fluor 350 hydrazide (Thermo Fisher, Cat. No. A-10439, 1:100 dilution of $2.5 \mathrm{mg} / \mathrm{ml}$ stock made up in PBS), Alexa Fluor 488 hydrazide (Cat. No. A-10436, 1:200 dilution of $0.5 \mathrm{mg} / \mathrm{ml}$ stock), Alexa Fluor 633 hydrazide (Cat. No. A-30634, 1:500 dilution of $0.5 \mathrm{mg} / \mathrm{ml}$ stock) or Alexa Fluor 647 hydrazide (Cat. No. A-20502, 1:200 dilution of 0.5 mg/ml stock) diluted in PBS with $0.1 \%$ Triton-X-100 for $1-3$ nights at $4^{\circ} \mathrm{C}$. Samples were washed in PBS with $0.1 \%$ Triton$\mathrm{X}-100$ four times for 30 minutes and cleared in BABB overnight or transferred to Cubic1 solution (93) right before imaging. Labeling of elastin by fluorescent hydrazide was verified by co-staining with rabbit anti-elastin antibodies (1:500, kindly provided by Robert Mecham) (see

Fig. S2C). For co-stains with antibodies, fluorescent hydrazide was added during incubation with secondary antibodies.

\section{Single-molecule in situ hybridization (smFISH)}

Postnatal lungs, inflated as described above, were fixed in $10 \%$ neutral buffered formalin (Fisher Scientific) for 24 hours at room temperature and transferred to $70 \%$ ethanol (made up in PBS) following 3 brief washes in PBS for embedding in paraffin. Sections were cut at $6 \mu \mathrm{m}$. smFISH was performed using a proprietary high-sensitivity RNA amplification and detection technology (RNAscope, Advanced Cell Diagnostics), according to the manufacturer's instructions using the indicated proprietary probes, the RNAscope Multiplex Fluorescent Reagent Kit (v.2) and TSA Plus reagents (Perkin Elmer; 1:500 dilution for Cy3 and Cy5 dyes) or Opal dyes (Akoya Biosciences, 1:500 dilution for Opal 520). After smFISH, sections were incubated in DAPI (used 
at $2 \mu \mathrm{g} / \mathrm{ml}$ in PBS) for $5 \mathrm{~min}$ to counterstain nuclei and mounted in Prolong Gold antifade reagent (Invitrogen). Proprietary (Advanced Cell Diagnostics) probes used were: Mm-Fgf18 (495421), Mm-Eln (319361), Mm-Acta2-C2 (319531-C2), tdTomato (317041-C3).

\section{Mosaic labeling and quantification of alveolar cells}

For labeling of individual AT1 cells, Hopx-CreER (80) or Foxa2-CreER (81) mice were crossed to Rosa26-mTmG (91) or Rosa26-Confetti (92) and dosed with 0.3-0.5 mg of tamoxifen 5 days prior to analysis at 2 months of age. Vibratome sections prepared from inflated and fixed lobes at $350 \mu \mathrm{m}$ thickness were stained with chicken anti-GFP (to visualize membrane targeted GFP in Rosa26-mTmG; ChromoTek) or rabbit anti-RFP (to visualize cytoplasmic RFP in Rosa26Confetti; Rockland) antibodies, Alexa Fluor 633 hydrazide and DAPI as described above, cleared and imaged in BABB. Confocal z-stacks were acquired using a 25x oil objective at an image resolution of $1,024 \times 1,024$ pixels and at a total thickness of 100-200 $\mu \mathrm{m}$, which allowed visualization of entire alveoli since the average diameter of a mouse alveolus is 50-60 $\mu \mathrm{m}(12)$. Imaris Software (Bitplane) was used for 3D visualization of individual AT1 cells and their arrangement in alveoli and for surface reconstruction and quantification of the number of AT1 cells in an alveolus. The entrance to individual alveoli or complexes was identified as the ring formed by thick elastic fibers, visualized by hydrazide (see Fig. 1, A, C and E, Fig. S2-S4, Movies S1 and S2). Although the arrangement of individual AT1 cells was found to be variable, the surface covered by individual cells approximated the mean alveolar surface area $\left(5,500 \mu \mathrm{m}^{2}\right)$ (12); hence the number of AT1 cells per alveolus is 1. Labeled AT1 cells in adult Foxa2-CreER; Rosa26-Confetti lungs were identified by immunostaining with rabbit anti-RFP (Rockland), Syrian hamster anti-Pdpn (DHSB) and rat anti-RAGE (R\&D) antibodies (see Fig. S5A). 
For mosaic labeling of capillary endothelial cells, VE-cadherin-CreER (83) (Fig. 1G and H) was bred to Rosa26-Confetti (92) and dosed with $1 \mathrm{mg}$ of tamoxifen at 3 weeks. Lungs were analyzed at 2 months. The cell type was inferred from cell size and shape. For specific labeling of aerocytes and general capillary cells, Apelin-CreER (84) (Fig. S6A) or Aplnr-CreER (85) (Fig. S6B) mice were bred to Rosa26-Confetti and dosed with tamoxifen (0.5 mg for Apelin-CreER, $0.1 \mathrm{mg}$ for Aplnr-CreER) at 2 months. Lungs were collected 5 days after tamoxifen injection. Vibratome sections (250 $\mu \mathrm{m}$ thickness) were stained with Alexa Fluor 633 hydrazide and endogenous RFP fluorescence was imaged in Cubic1-cleared sections, or stained with rabbit anti-RFP (Rockland) and Armenian hamster anti-Pecam1 (Bio-Rad) antibodies and fluorescent hydrazide as described above and cleared and imaged in BABB to visualize individual endothelial cells and their arrangement in the alveolar capillary network in Imaris. For quantification of the number of endothelial cells per alveolus, vibratome sections were stained with rabbit anti-Erg antibodies (1:100, Abcam, Cat. No. ab110639) to identify endothelial nuclei and fluorescent hydrazide to visualize elastic fibers. Endothelial nuclei and alveolar entrance rings were quantified in the Surpass view of Imaris.

For labeling of pericytes, Pdgfra-CreER (86); Rosa26-mTmG (Fig. 1I and Fig. S5E) or Notch3-CreER (88); Rosa26-Confetti lungs (Fig. S6C) were dosed with tamoxifen (2 mg for Pdgfra-CreER; $0.5 \mathrm{mg}$ for Notch3-CreER) at 3 months. Lungs were collected at 5 days after tamoxifen injection and vibratome sections $(350 \mu \mathrm{m})$ were stained with rat anti-GFP (to visualize membrane targeted GFP in Rosa26-mTmG; ChromoTek) or rabbit anti-RFP (to visualize cytoplasmic RFP in Rosa26-Confetti; Rockland) antibodies, rabbit anti-NG2 (Millipore) or rat anti-Pdgfrb (eBioscience) antibodies, and Alexa Fluor 633 hydrazide, cleared and imaged in $\mathrm{BABB}$, and analyzed in Imaris. Pericytes were identified by NG2 or Pdgfrb co-staining. The 
transgenic Pdgfra-CreER strain labels multiple populations of lung mesenchyme including pericytes, as reported for the brain (86).

For labeling of alveolar fibroblasts, Gli1-CreER (89); Rosa26-mTmG (Fig. 1J and Fig. S5F) or Pdgfra-CreER (87); Rosa26-Confetti lungs (Fig. S6D) were dosed with tamoxifen (4 mg for Gli1-CreER; $0.5 \mathrm{mg}$ for Pdgfra-CreER) at 2 months. Lungs were collected at 5 days after tamoxifen injection and vibratome sections (350 $\mu \mathrm{m}$ thickness) were stained with rat anti-GFP and goat anti-Itga8 (Integrin $\alpha 8)$ antibodies (R\&D) to identify alveolar fibroblasts $(53,62)$ and with Alexa Fluor 633 hydrazide, cleared and imaged in BABB, and analyzed in Imaris.

For multicolor labeling of myofibroblasts, SMMHC-CreER (64) mice were bred to the multicolor Cre reporter Rosa26-Rainbow (65) and dosed with $0.1 \mathrm{mg}$ tamoxifen at P5. Lungs were collected and stained with Alexa Fluor 647 hydrazide at P10, and endogenous Cerulean, mCherry and mOrange fluorescence was imaged in vibratome sections (250 $\mu \mathrm{m}$ thickness) cleared with ScaleA2 (incubation for 1-3 days at $4^{\circ} \mathrm{C}$ ) (94). For quantification of myofibroblasts, vibratome sections from P10 Lgr6-CreER (60); Rosa26-tdTomato lungs were stained with rabbit anti-RFP (Rockland) and mouse anti-SMA antibodies (Sigma) and fluorescent hydrazide, cleared and imaged in $\mathrm{BABB}$, and analyzed in Imaris. Each intersection of neighboring alveoli was found to be occupied by a myofibroblast (identified by the lineage tag, expression of SMA and alignment with elastic fibers) and the cells form a ring around the alveolar entrance with no gaps. Each alveolus has 3-5 alveolar neighbors and is surrounded by 3-5 myofibroblasts.

For labeling of AT2 cells, Shh-GFP-Cre (82) mice were bred to Rosa26-mTmG (91) and analyzed at 2 months by immunostaining with chicken anti-GFP antibodies (Abcam) and Alexa Fluor 647 hydrazide, cleared and imaged in BABB. Clonal labeling was not necessary for AT2 cells because of their simple, well defined shape. For quantification of the number of AT2 cells 
per alveolus, vibratome sections $(500 \mu \mathrm{m})$ from wild-type (C57BL/6) lungs were stained with rabbit anti-SftpC (Millipore) and Armenian hamster anti-Muc1 (Thermo Fisher) antibodies (to visualize AT2 cells), fluorescent hydrazide (to visualize elastic fibers and count alveolar units), and DAPI (to identify nuclei) as described above, and cleared and imaged in BABB. Confocal zstacks taken were taken at an optical section thickness of $1 \mu \mathrm{m}$ and a total thickness of $150 \mu \mathrm{m}$ with a 25x oil objective, and the number of AT2 cells and gas exchange units (simple alveoli or individual units of alveolar complexes) were quantified in $\mathrm{z}$-stacks ( $\mathrm{n}=3$ mice, one $\mathrm{z}$-stack per mouse) in the Surpass view of Imaris. The number of AT2 cells (SftpC+ Muc1+ cells) was counted using the Imaris spot detection tool (stack 1: 338 AT2 cells, stack 2: 297 AT2 cells, stack 3: 370 AT2 cells) and the number of gas exchange units (the sum of the number of thick fiber rings at the entrance to simple alveoli and alveolar complexes and the number of thin fibers that subdivide alveolar complexes) was counted using the Imaris measurement point tool (stack 1: 300 gas exchange units, stack 2: 284 units, stack 3: 294 units). The average number of AT2 cells per gas exchange unit was calculated from these measurements (mean \pm s.d., 1.14 \pm 0.1 AT2 cells).

For quantification of the proportion of simple alveoli and alveolar complexes, 3 monthold C56BL/6 lungs ( $\mathrm{n}=3$ ) were stained with fluorescent hydrazide and $\mathrm{z}$-stacks were taken at a thickness of $300 \mu \mathrm{m}$ with a $25 \mathrm{x}$ oil objective. The elastic fiber network was visualized in three dimensions in the Surpass view of Imaris. The entrance to individual alveoli and complexes was identified as the ring formed by thick elastic fibers, visualized by hydrazide. Simple alveoli were scored as units that contain only thick fibers. Alveolar complexes were scored as units that also contain thin elastic fibers anchored on thick fiber rings. The number of thick fiber rings with and 
without thin fibers was quantified in $\mathrm{z}$-stacks $(\mathrm{n}=3)$, corresponding to the number of alveolar complexes and simple alveoli, respectively.

For analysis of the structure of alveolar complexes, the number of alveolar epithelial cells was scored in adult Foxa2-CreER; Rosa26-mTmG lungs at 3 months stained with rabbit antiSftpC (Millipore) and rat anti-RAGE (R\&D) antibodies to visualize AT2 and AT1 cells (see Fig. S5A), respectively, fluorescent hydrazide to visualize elastic fibers and Dapi to identify nuclei. The number of AT1 and AT2 cells was scored in 2-unit $(n=7)$ and 4-unit complexes $(n=4)$ from 3D renderings of confocal z-stacks in the Surpass view of Imaris by counting alveolar epithelial nuclei.

\section{Mapping respiratory airways and alveolar development}

The boundary between conducting and respiratory airways was mapped on the L.L1.A3 branch lineage (56). L.L1.A3 daughters are located at the flat edge of the left lobe (see Fig. S7A), which allowed imaging of whole lobes by confocal microscopy without sectioning. Conducting airways were defined as the generations of airways lined by a pseudostratified or columnar epithelium made up of Sox2-expressing airway epithelial cells. Respiratory airways were defined as the generations of airways lined by an epithelium made up of Sox2-negative alveolar epithelial cells (AT1, AT2 cells). The first-order respiratory airway (transitional bronchiole) was identified as a hybrid tube composed of a conducting and a respiratory part (1). In mice, the transition from conducting to respiratory airways is abrupt (57). The compartment boundary was identified by Sox 2 immunostaining (57) as described above or by fluorescent streptavidin staining (58) to detect biotin expressed by airway epithelial cells: whole left lobes, dissected and fixed as described above in 4\% PFA, were incubated in fluorescent streptavidin (Alexa Fluor 568 conjugate, Thermo Fisher, Cat. No. S-11226) diluted 1:200 in block (5\% goat serum with 0.5\% 
Triton X-100 and 3\% BSA in PBS) for 3 nights at $4^{\circ} \mathrm{C}$. Samples were washed in PBS with $0.1 \%$ Triton-X-100 five times for 1 hour and cleared and imaged in BABB. For co-stains with antibodies, fluorescent streptavidin was added during incubation with primary antibodies.

Alveolar development was mapped on respiratory airways of the first 2-3 generations (L.L1.A3 lineage), since branching in early generations is less variable and these airways form in the embryonic lung (56), which allows analysis of alveolar development without inflation. We analyzed stalks (rather than branch tips) to map the position of epithelial cells and smooth muscle cells relative to the axis of the airway tube.

\section{Lineage tracing of airway smooth muscle}

Lgr6-EGFP-IRES-CreERT2 (Lgr6-CreER) (60) males were bred to Rosa26-tdTomato (61)

females to generate heterozygous Lgr6-CreER males homozygous for Rosa26-tdTomato. The males were bred to CD1 wild-type females (Charles River Laboratories), since they were found to tolerate higher doses of tamoxifen than C57BL/6. Pregnant females from these crosses were dosed at E14.5 with saturating (4 mg) tamoxifen to label smooth muscle on embryonic airways with heritable tdTomato expression and the labeling was analyzed at E16.5, E17.5, P10, and P60 (Fig. S10E) by staining whole left lobes dissected and fixed as described above with rabbit antiRFP (Rockland) and mouse anti-SMA (Sigma) antibodies, fluorescent streptavidin and hydrazide as described above, cleared in BABB and imaged on a Zeiss 880 confocal laser scanning microscope using a long working distance objective (LD Plan-Apochromat 20x/1.0 Corr M32 $85 \mathrm{~mm})$. 


\section{Clonal analysis of smooth muscle}

SMMHC-CreER (64) mice were crossed to the multicolor Cre reporter Rosa26-Rainbow (65). Pregnant (E15) females received intraperitoneal injections of limiting doses $(0.05 \mathrm{mg})$ of tamoxifen dissolved in corn oil to induce rare recombination events, which generated wellseparated clones of cells that express one of three fluorescent proteins.

Postnatal (P10) lungs were perfused with PBS, inflated with 2\% low-melting point agarose and fixed in $4 \%$ PFA for 2 hours at $4^{\circ} \mathrm{C}$. Vibratome sections were prepared from left or right cranial lobes at 200-300 $\mu \mathrm{m}$ thickness. Sections were incubated with Alexa Fluor 350 hydrazide and SMA-Cy5 in PBS with 5\% goat serum and $0.5 \%$ Triton X-100 for 2 nights at $4^{\circ} \mathrm{C}$, washed in PBS with $0.1 \%$ Tween-20 five times for $15-30$ minutes at $4{ }^{\circ} \mathrm{C}$ and transferred to Cubic1 solution right before imaging. Endogenous Cerulean, mCherry and mOrange fluorescence was imaged on a Zeiss 880 confocal laser scanning microscope equipped with a 440 nm laser to excite Cerulean.

\section{Analysis of Notch activity in alveolar epithelial progenitors}

Embryonic (E16.5-E18.5) CBF1:H2B-Venus (71) lungs were dissected and fixed in 4\% PFA for 1-2 hours. Whole lobes were immunostained as above with chicken anti-GFP (Abcam), rabbit anti-Fgfr2 (Santa Cruz), rat anti-E-cadherin (Thermo Fisher) and Armenian hamster anti-Pecam1 (Bio-Rad) antibodies, cleared and imaged in BABB.

For Hes1 antibody stains, lungs were fixed in 0.5\% PFA for 3 hours at room temperature, washed 3 times in PBS and incubated in $30 \%$ sucrose overnight at $4^{\circ} \mathrm{C}$. Left lobes were embedded in optimal cutting temperature (OCT) compound and sectioned at $30 \mu \mathrm{m}$ thickness. Cryosections were washed in PBS for 5 minutes and permeabilized in $0.3 \%$ Triton-X-100 for 1015 minutes at room temperature. Sections were washed again in PBS for 5 minutes, blocked in 
$10 \%$ goat serum in PBS with $0.1 \%$ Tween-20 for 1-2 hours at room temperature, and incubated in primary antibodies diluted in block overnight at $4^{\circ} \mathrm{C}$. Primary antibodies used, at indicated concentrations, were: rabbit anti-Hes1 (1:200, Cell Signaling, Cat. No. 11988), chicken anti-GFP (1:1000, Abcam, Cat. No. ab13970), Syrian hamster anti-podoplanin (Pdpn; 1:50, DHSB, Cat. No. 8.1.1), rat anti-RAGE (1:500, R\&D Systems, Cat. No. MAB1179, clone 175410) and rat anti-E-cadherin (1:1000, Thermo Fisher, Cat. No. 13-1900). Sections were washed in PBS with 0.1\% Tween-20 four times for 5 minutes at room temperature and incubated in secondary antibodies and DAPI (1:500 dilution of $1 \mathrm{mg} / \mathrm{ml}$ stock) diluted in block overnight at $4{ }^{\circ} \mathrm{C}$. Secondary antibodies used, at indicated concentrations, were: HRP-conjugated goat anti-rabbit IgG (1:150, Vector, Cat. No. PI-1000), goat anti-chicken Alexa Fluor 488 (1:500, Thermo Fisher, Cat. No. A-11039), goat anti-Syrian hamster Alexa Fluor 647 (1:500, Thermo Fisher, Cat. No. A-21451), donkey anti-rat Alexa Fluor 488 (1:500, Thermo Fisher, Cat. No. A-21208). Sections were washed again as above and incubated in Cy3 tyramide reagent (Perkin Elmer, Cat. No. SAT704A001EA) diluted 1:100 in amplification diluent for 10 minutes at room temperature. Sections were washed in PBS four times for 15 minutes, mounted in Prolong Gold antifade mountant (Thermo Fisher) and imaged on a Zeiss 780 confocal laser scanning microscope.

\section{Pharmacologic Notch inhibition in embryonic lungs}

The $\gamma$-secretase inhibitor DBZ (Tocris, Cat. No. 4489) was delivered by daily intraperitoneal injection in timed-pregnant (E16-E18) C57BL/6 females at $30 \mu \mathrm{mol}$ per kilogram body weight. A $100 \mathrm{mM}$ DBZ stock was prepared in DMSO and diluted to a volume of $200 \mu 1$ in corn oil. Controls were injected with an equivalent volume of DMSO diluted in corn oil. Lungs were dissected at E18.5 and fixed in $4 \%$ PFA for 2 hours at $4^{\circ} \mathrm{C}$, immunostained for rabbit anti-SftpC (Millipore), Syrian hamster anti-Pdpn (DSHB) and rat anti-E-cadherin (Thermo Fisher) 
antibodies as described above, cleared and imaged in BABB on a Zeiss 780 confocal laser scanning microscope. 500 AT2 cells were scored for each condition (vehicle control, DBZ) in six biological replicates ( $\mathrm{n}=6$ mice per treatment group). For quantification of AT2 cell proliferation, lungs were immunostained with anti-SftpC and anti-Ki67-eFluor 570 antibodies $(1: 150$, eBioscience, Cat. No. 41-5698-82) as above. AT2 cells $(n=500)$ were scored for each condition (vehicle control, DBZ) in three biological replicates ( $\mathrm{n}=3$ mice per treatment group).

\section{Notch inhibition in cultured alveolar epithelial progenitors}

Epithelial progenitors were isolated as previously described (40) from E16.5 C57BL/6 lungs pooled by litter and cultured on a layer of growth factor reduced Matrigel (BD) in serum-free media (DMEM/F12 supplemented with L-glutamine and penicillin-streptomycin) with added FGF ligands (Fgf7; $4 \mu \mathrm{M}, \mathrm{R} \& \mathrm{D}$ Systems, Cat. No. 5028-KG-025), the $\gamma$-secretase inhibitor DAPT (10 $\mu \mathrm{M}$; Tocris, Cat. No. 2634), or Notch1 or Notch 2 antibody antagonists (75) (kindly provided by Chris Siebel, Genentech Inc.; $20 \mu \mathrm{g}$ of antibody per $\mathrm{ml}$ of culture). Cultures were fixed after 4 days, immunostained for rabbit anti-SftpC (Millipore) and rat anti-RAGE antibodies (R\&D) as described above to identify alveolar epithelial progenitors (SftpC+ RAGE+), AT1 cells (SftpC- RAGE+) and AT2 cells (SftpC+ RAGE-), and mounted in Vectashield media with DAPI (Vector) for imaging on a Zeiss 780 confocal laser scanning microscope. 500 cells were scored for each condition (Fgf7 only control, Fgf7 with DAPT, Fgf7 with anti-Notch1 antibody, Fgf7 with anti-Notch2 antibody) in three biological replicates (independent cultures established from 3 litters). 


\section{Confocal imaging}

Images were acquired using inverted Zeiss 780 or upright Zeiss 880 confocal laser scanning microscopes. Zen Imaging Software (Zeiss) and Adobe Photoshop were used to adjust image levels and pseudocolor the images. Volocity Software (Perkin Elmer) was used to generate maximum intensity projections from z-stacks. The fine filter was applied to images of Sox2 antibody stained lungs. Imaris Software (Bitplane) was used for 3D visualization.

\section{Analysis of single-cell RNA-sequencing (scRNAseq) data}

Processed scRNAseq MARS-Seq data for developing (E16.5, E18.5) mouse lung by Cohen et al. (74) were obtained from GEO (accession number GSE119228) as gene count tables with demultiplexed and aligned reads and expression profiles of cells were clustered using the $\mathrm{R}$ software package Seurat (version 2.3) (95). In brief, cells with fewer than 500 unique molecular identifiers were excluded and data were log-transformed: $\ln$ (unique molecular identifiers per ten thousand+1) $[\ln (\mathrm{UP} 10 \mathrm{~K}+1)]$. Highly variable genes were selected using the 'FindVariableGenes' function (dispersion (mean/variance) z-score > 0.5) for linear dimensionality reduction using principal component analysis. The number of principal components was selected by inspection of the plot of variance explained. Cells were clustered by constructing a shared nearest neighbor graph and clusters were visualized by t-distributed stochastic neighbor embedding (tSNE). Lung epithelial, endothelial, and stromal cells were identified by $C d h 1, C d h 5$ or Collal expression, respectively. AT2 cells were identified by Etv5, Sox9, Fgfr2, Lamp3 and Sftpal expression. AT1 cells were identified by Ager, Hopx, Pdpn and Aqp5 expression. Alveolar epithelial progenitor cells were identified by co-expression of AT2 (Etv5, Sox9, Fgfr2, Lamp3, Sftpal) and AT1 markers (Ager, Hopx, Pdpn) (40, 47). Heatmaps were generated with the 'heatmap.3' function. Analysis of scRNAseq data for E18.5 mouse lung cells (Treutlein et al.) was conducted as 
previously described (47). Processed scRNAseq Smart-Seq2 data for adult mouse lung (Tabula Muris Senis) were obtained from Synapse (Synapse ID: syn21041850) $(62,63)$ as a Seurat object with annotated cell types. Dot plots were generated using the 'DotPlot' function in Seurat.

\section{Statistics and reproducibility}

Data analysis and statistical tests were performed using R software (version 3.5.1). Data are represented as mean \pm standard deviation (s.d.). For comparison of two groups, a two-sided Wilcoxon rank sum test was conducted at 5\% significance level. Replicate experiments are biological replicates with different animals. For all graphs, the number of biologically independent samples is reported in the Figure Legends. Sample size calculations were not performed. Mice of the appropriate age were allocated into experimental groups (control vs. treatment) at random. The investigators were not blinded to sample allocation. 


\section{References}

1. M. Rodriguez, S. Bur, A. Favre, E. R. Weibel, Pulmonary acinus: geometry and morphometry of the peripheral airway system in rat and rabbit. Am J Anat 180, 143-155 (1987).

2. B. Haefeli-Bleuer, E. R. Weibel, Morphometry of the human pulmonary acinus. Anat Rec 220, 401-414 (1988).

3. E. R. Weibel, The pathway for oxygen. Structure and function in the mammalian respiratory system. (Harvard University Press, Cambridge, Massachusetts, 1984).

4. R. R. Mercer, J. D. Crapo, Three-dimensional reconstruction of the rat acinus. J Appl Physiol 63, 785-794 (1987).

5. E. A. Boyden, The structure of the pulmonary acinus in a child of six years and eight months. Am J Anat 132, 275-299 (1971).

6. K. K. Pump, The morphology of the finer branches of the bronchial tree of the human lung. Dis Chest 46, 379-398 (1964).

7. K. K. Pump, Morphology of the acinus of the human lung. Dis Chest 56, 126-134 (1969).

8. J. E. Hansen, E. P. Ampaya, G. H. Bryant, J. J. Navin, Branching pattern of airways and air spaces of a single human terminal bronchiole. J Appl Physiol 38, 983-989 (1975).

9. M. Ochs et al., The number of alveoli in the human lung. Am J Respir Crit Care Med 169, 120-124 (2004).

10. J. Knust, M. Ochs, H. J. Gundersen, J. R. Nyengaard, Stereological estimates of alveolar number and size and capillary length and surface area in mice lungs. Anat Rec 292, 113122 (2009).

11. D. M. Hyde, N. K. Tyler, L. F. Putney, P. Singh, H. J. Gundersen, Total number and mean size of alveoli in mammalian lung estimated using fractionator sampling and unbiased estimates of the Euler characteristic of alveolar openings. Anat Rec A Discov Mol Cell Evol Biol 277, 216-226 (2004).

12. R. R. Mercer, M. L. Russell, J. D. Crapo, Alveolar septal structure in different species. $J$ Appl Physiol 77, 1060-1066 (1994). 
13. J. P. Schneider, C. Wrede, C. Muhlfeld, The three-dimensional ultrastructure of the human alveolar epithelium revealed by focused ion beam electron microscopy. Int J Mol Sci 21, 1-19 (2020).

14. P. Gehr, M. Bachofen, E. R. Weibel, The normal human lung: ultrastructure and morphometric estimation of diffusion capacity. Respir Physiol 32, 121-140 (1978).

15. F. N. Low, The pulmonary alveolar epithelium of laboratory mammals and man. Anat $\operatorname{Rec} 117,241-263$ (1953).

16. J. D. Crapo et al., Morphometric characteristics of cells in the alveolar region of mammalian lungs. Am Rev Respir Dis 128, S42-46 (1983).

17. E. R. Weibel, The mystery of "non-nucleated plates" in the alveolar epithelium of the lung explained. Acta Anat 78, 425-443 (1971).

18. E. R. Weibel, Morphological basis of alveolar-capillary gas exchange. Physiol Rev 53, 419-495 (1973).

19. C. M. Alvira, R. E. Morty, Can we understand the pathobiology of bronchopulmonary dysplasia? J Pediatr 190, 27-37 (2017).

20. M. B. Drummond, A. S. Buist, J. D. Crapo, R. A. Wise, S. I. Rennard, Chronic obstructive pulmonary disease: NHLBI Workshop on the primary prevention of chronic lung diseases. Ann Am Thorac Soc 11, Suppl 3, S154-160 (2014).

21. T. E. King, A. Pardo, M. Selman, Idiopathic pulmonary fibrosis. Lancet 378, 1949-1961 (2011).

22. T. J. Franks et al., Lung pathology of severe acute respiratory syndrome (SARS): a study of 8 autopsy cases from Singapore. Hum Pathol 34, 743-748 (2003).

23. D. M. Hwang et al., Pulmonary pathology of severe acute respiratory syndrome in Toronto. Mod Pathol 18, 1-10 (2005).

24. Z. Xu et al., Pathological findings of COVID-19 associated with acute respiratory distress syndrome. Lancet Respir Med 8, 420-422 (2020).

25. R. W. Amy, D. Bowes, P. H. Burri, J. Haines, W. M. Thurlbeck, Postnatal growth of the mouse lung. J Anat 124, 131-151 (1977).

26. H. Bostrom et al., PDGF-A signaling is a critical event in lung alveolar myofibroblast development and alveogenesis. Cell 85, 863-873 (1996). 
27. P. H. Burri, Fetal and postnatal development of the lung. Annu Rev Physiol 46, 617-628 (1984).

28. P. H. Burri, Postnatal development and growth in The Lung: Scientific Foundations 2nd ed. (eds. R. G. Crystal, J. B. West, E. R. Weibel \& P. J. Barnes) (Lippincott-Raven, Philadelphia, 1997).

29. C. Gaultier, A. Denjean, Developmental anatomy and physiology of the respiratory system in Pediatric Respiratory Medicine 2nd ed. (eds. L. M. Taussig \& L. I. Landau) (Mosby Elsevier, Philadelphia, 2008).

30. A. A. Hislop, J. S. Wigglesworth, R. Desai, Alveolar development in the human fetus and infant. Early Hum Dev 13, 1-11 (1986).

31. W. M. Thurlbeck, Postnatal growth and development of the lung. Am Rev Respir Dis 111, 803-844 (1975).

32. S. Kotecha, Lung growth: implications for the newborn infant. Arch Dis Child Fetal Neonatal Ed 82, F69-74 (2000).

33. M. S. Dunnill, Postnatal growth of the lung. Thorax 17, 329-333 (1962).

34. Y. Kapanci, A. Assimacopoulos, C. Irle, A. Zwahlen, G. Gabbiani, "Contractile interstitial cells" in pulmonary alveolar septa: a possible regulator of ventilationperfusion ratio? Ultrastructural, immunofluorescence, and in vitro studies. J Cell Biol 60, 375-392 (1974).

35. K. O. Leslie, J. J. Mitchell, J. L. Woodcock-Mitchell, R. B. Low, Alpha smooth muscle actin expression in developing and adult human lung. Differentiation 44, 143-149 (1990).

36. K. Branchfield et al., A three-dimensional study of alveologenesis in mouse lung. Dev Biol 409, 429-441 (2016).

37. M. Endale et al., Temporal, spatial, and phenotypical changes of PDGFRalpha expressing fibroblasts during late lung development. Dev Biol 425, 161-175 (2017).

38. A. Moiseenko et al., Origin and characterization of alpha smooth muscle actin-positive cells during murine lung development. Stem Cells 35, 1566-1578 (2017).

39. R. Li et al., Pdgfra marks a cellular lineage with distinct contributions to myofibroblasts in lung maturation and injury response. eLife 7, e36865 (2018). 
40. T. J. Desai, D. G. Brownfield, M. A. Krasnow, Alveolar progenitor and stem cells in lung development, renewal and cancer. Nature 507, 190-194 (2014).

41. D. B. Frank et al., Emergence of a wave of Wnt signaling that regulates lung alveologenesis by controlling epithelial self-renewal and differentiation. Cell Rep 17, 2312-2325 (2016).

42. J. Yang et al., The development and plasticity of alveolar type 1 cells. Development 143, 54-65 (2016).

43. U. Laresgoiti et al., Lung epithelial tip progenitors integrate glucocorticoid- and STAT3mediated signals to control progeny fate. Development 143, 3686-3699 (2016).

44. B. L. Hogan et al., Repair and regeneration of the respiratory system: complexity, plasticity, and mechanisms of lung stem cell function. Cell Stem Cell 15, 123-138 (2014).

45. J. A. Zepp et al., Genomic, epigenomic, and biophysical cues controlling the emergence of the lung alveolus. Science 371, eabc3172 (2021).

46. J. Li et al., The strength of mechanical forces determines the differentiation of alveolar epithelial cells. Dev Cell 44, 297-312 (2018).

47. B. Treutlein et al., Reconstructing lineage hierarchies of the distal lung epithelium using single-cell RNA-seq. Nature 509, 371-375 (2014).

48. J. A. Pierce, R. V. Ebert, Fibrous network of the lung and its change with age. Thorax 20, 469-476 (1965).

49. F. Orsós, Über das elastische Gerüst der normalen und der emphysematösen Lunge. Beiträge zur pathologischen Anatomie und zur allgemeinen Pathologie 41, 95-121 (1907).

50. Z. Shen, Z. Lu, P. Y. Chhatbar, P. O'Herron, P. Kara, An artery-specific fluorescent dye for studying neurovascular coupling. Nat Methods 9, 273-276 (2012).

51. V. E. Krahl, Current concept of the finer structure of the lung. AMA Arch Intern Med 96, 342-356 (1955).

52. C. Desplechain, B. Foliguet, E. Barrat, G. Grignon, F. Touati, Les pores de Kohn des alvéoles pulmonaires. Bull Eur Physiopathol Respir 19, 59-68 (1983).

53. A. Gillich et al., Capillary cell-type specialization in the alveolus. Nature 586, 785-789 (2020). 
54. L. Vila Ellis et al., Epithelial Vegfa specifies a distinct endothelial population in the mouse lung. Dev Cell 52, 617-630 (2020).

55. K. C. Stone, R. R. Mercer, P. Gehr, B. Stockstill, J. D. Crapo, Allometric relationships of cell numbers and size in the mammalian lung. Am J Respir Cell Mol Biol 6, 235-243 (1992).

56. R. J. Metzger, O. D. Klein, G. R. Martin, M. A. Krasnow, The branching programme of mouse lung development. Nature 453, 745-750 (2008).

57. D. Martinez Alanis, D. R. Chang, H. Akiyama, M. A. Krasnow, J. Chen, Two nested developmental waves demarcate a compartment boundary in the mouse lung. Nat Commun 5, Article 3923 (2014).

58. G. D. Scott, E. D. Blum, A. D. Fryer, D. B. Jacoby, Tissue optical clearing, threedimensional imaging, and computer morphometry in whole mouse lungs and human airways. Am J Respir Cell Mol Biol 51, 43-55 (2014).

59. M. E. Kumar et al., Mesenchymal cells. Defining a mesenchymal progenitor niche at single-cell resolution. Science 346, 1258810-1 to 9 (2014).

60. H. J. Snippert et al., Lgr6 marks stem cells in the hair follicle that generate all cell lineages of the skin. Science 327, 1385-1389 (2010).

61. L. Madisen et al., A robust and high-throughput Cre reporting and characterization system for the whole mouse brain. Nat Neurosci 13, 133-140 (2010).

62. K. J. Travaglini et al., A molecular cell atlas of the human lung from single-cell RNA sequencing. Nature 587, 619-625 (2020).

63. Tabula Muris Consortium, A single-cell transcriptomic atlas characterizes ageing tissues in the mouse. Nature 583, 590-595 (2020).

64. A. Wirth et al., G12-G13-LARG-mediated signaling in vascular smooth muscle is required for salt-induced hypertension. Nat Med 14, 64-68 (2008).

65. Y. Rinkevich, P. Lindau, H. Ueno, M. T. Longaker, I. L. Weissman, Germ-layer and lineage-restricted stem/progenitors regenerate the mouse digit tip. Nature 476, 409-413 (2011).

66. A. S. Hagan, B. Zhang, D. M. Ornitz, Identification of a FGF18-expressing alveolar myofibroblast that is developmentally cleared during alveologenesis. Development 147, (2020). 
67. D. G. Brownfield, A. Gillich, A. Diaz de Arce, T. Desai, M. A. Krasnow, Alveolar cell fate selection and lifelong maintenance by FGF signaling. In review.

68. K. G. Guruharsha, M. W. Kankel, S. Artavanis-Tsakonas, The Notch signalling system: recent insights into the complexity of a conserved pathway. Nature Rev Genet 13, 654666 (2012).

69. K. Xu et al., Lunatic Fringe-mediated Notch signaling is required for lung alveogenesis. Am J Physiol Lung Cell Mol Physiol 298, L45-56 (2010).

70. J. Finn et al., Dlk1-mediated temporal regulation of Notch signaling is required for differentiation of alveolar type II to type I cells during repair. Cell Rep 26, 2942-2954 e2945 (2019).

71. S. Nowotschin, P. Xenopoulos, N. Schrode, A. K. Hadjantonakis, A bright single-cell resolution live imaging reporter of Notch signaling in the mouse. BMC Dev Biol 13, Article 15 (2013).

72. J. Milano et al., Modulation of Notch processing by gamma-secretase inhibitors causes intestinal goblet cell metaplasia and induction of genes known to specify gut secretory lineage differentiation. Toxicol Sci 82, 341-358 (2004).

73. P. N. Tsao et al., Epithelial Notch signaling regulates lung alveolar morphogenesis and airway epithelial integrity. Proc Natl Acad Sci U S A 113, 8242-8247 (2016).

74. M. Cohen et al., Lung single-cell signaling interaction map reveals basophil role in macrophage imprinting. Cell 175, 1031-1044 (2018).

75. D. Lafkas et al., Therapeutic antibodies reveal Notch control of transdifferentiation in the adult lung. Nature 528, 127-131 (2015).

76. A. S. Ghabrial, M. A. Krasnow, Social interactions among epithelial cells during tracheal branching morphogenesis. Nature 441, 746-749 (2006).

77. O. Narvaez del Pilar, Chen, J., Three-axis classification of mouse lung mesenchymal cells reveals two populations of myofibroblasts doi: https://doi.org/10.1101/2021.08.03.454930. bioRxiv (2021).

78. J. D. Crapo, B. E. Barry, P. Gehr, M. Bachofen, E. R. Weibel, Cell number and cell characteristics of the normal human lung. Am Rev Respir Dis 125, 740-745 (1982).

79. J. P. Kinsella, A. Greenough, S. H. Abman, Bronchopulmonary dysplasia. Lancet 367, 1421-1431 (2006). 
80. N. Takeda et al., Interconversion between intestinal stem cell populations in distinct niches. Science 334, 1420-1424 (2011).

81. E. J. Park et al., System for tamoxifen-inducible expression of cre-recombinase from the Foxa2 locus in mice. Dev Dyn 237, 447-453 (2008).

82. B. D. Harfe et al., Evidence for an expansion-based temporal Shh gradient in specifying vertebrate digit identities. Cell 118, 517-528 (2004).

83. A. Monvoisin et al., VE-cadherin-CreERT2 transgenic mouse: a model for inducible recombination in the endothelium. Dev Dyn 235, 3413-3422 (2006).

84. X. Tian et al., Subepicardial endothelial cells invade the embryonic ventricle wall to form coronary arteries. Cell Res 23, 1075-1090 (2013).

85. H. I. Chen et al., The sinus venosus contributes to coronary vasculature through VEGFCstimulated angiogenesis. Development 141, 4500-4512 (2014).

86. S. H. Kang, M. Fukaya, J. K. Yang, J. D. Rothstein, D. E. Bergles, NG2+ CNS glial progenitors remain committed to the oligodendrocyte lineage in postnatal life and following neurodegeneration. Neuron 68, 668-681 (2010).

87. M. I. Chung, M. Bujnis, C. E. Barkauskas, Y. Kobayashi, B. L. M. Hogan, Nichemediated BMP/SMAD signaling regulates lung alveolar stem cell proliferation and differentiation. Development 145, 1-10 (2018).

88. S. Fre et al., Notch lineages and activity in intestinal stem cells determined by a new set of knock-in mice. PLoS One 6, e25785 (2011).

89. S. Ahn, A. L. Joyner, Dynamic changes in the response of cells to positive hedgehog signaling during mouse limb patterning. Cell 118, 505-516 (2004).

90. O. Wendling, J. M. Bornert, P. Chambon, D. Metzger, Efficient temporally-controlled targeted mutagenesis in smooth muscle cells of the adult mouse. Genesis 47, 14-18 (2009).

91. M. D. Muzumdar, B. Tasic, K. Miyamichi, L. Li, L. Luo, A global double-fluorescent Cre reporter mouse. Genesis 45, 593-605 (2007).

92. H. J. Snippert et al., Intestinal crypt homeostasis results from neutral competition between symmetrically dividing Lgr5 stem cells. Cell 143, 134-144 (2010). 
93. E. A. Susaki et al., Whole-brain imaging with single-cell resolution using chemical cocktails and computational analysis. Cell 157, 726-739 (2014).

94. H. Hama et al., Scale: a chemical approach for fluorescence imaging and reconstruction of transparent mouse brain. Nat Neurosci 14, 1481-1488 (2011).

95. A. Butler, P. Hoffman, P. Smibert, E. Papalexi, R. Satija, Integrating single-cell transcriptomic data across different conditions, technologies, and species. Nat Biotechnol 36, 411-420 (2018).

96. C. M. Chao, E. El Agha, C. Tiozzo, P. Minoo, S. Bellusci, A breath of fresh air on the mesenchyme: impact of impaired mesenchymal development on the pathogenesis of bronchopulmonary dysplasia. Front Med 2, Article 27 (2015).

97. C. G. Loosli, E. L. Potter, Pre- and postnatal development of the respiratory portion of the human lung with special reference to the elastic fibers. Am Rev Respir Dis 80, 5-23 (1959).

98. S. J. Bray, S. Takada, E. Harrison, S. C. Shen, A. C. Ferguson-Smith, The atypical mammalian ligand Delta-like homologue 1 (Dlk1) can regulate Notch signalling in Drosophila. BMC Dev Biol 8, Article 11 (2008). 
Gillich et al., p. 43

\section{Acknowledgements}

We thank C. Siebel and R. Mecham for reagents; J. Campbell for help with culture experiments; Maya Kumar for advice and discussion; M. Petersen for help with illustrations; the Department of Comparative Medicine Animal Histology Services for technical assistance; M. L. IruelaArispe, K. Red-Horse, I. L. Weissman and B. Zhou for sharing mouse lines; and members of the Krasnow lab for comments on the manuscript. This work was supported by the Vera Moulton Wall Center for Pulmonary Vascular Disease at Stanford and grants from the Austrian Science Fund (J-3373) and the American Heart Association (16POST27250261) to A.G. K.J.T. was supported by a Paul and Mildred Berg Stanford Graduate Fellowship. M.A.K. is an investigator of the Howard Hughes Medical Institute.

\section{Author Contributions}

A.G. and M.A.K. conceived, designed, and analyzed experiments and wrote the manuscript. A.G. performed the experiments. A.G. and K.R.St.J. performed lineage tracing. D.G.B. performed culture experiments. A.G. and D.G.B. analyzed scRNAseq data. A.G., K.J.T., and R.J.M performed mosaic labeling. R.J.M. provided guidance on the project. All authors reviewed the manuscript.

\section{List of Supplementary Materials}

Figures S1-S17

Table S1. Cre and CreER drivers used for clonal labeling and lineage tracing.

Table S2. Airway clones generated by tamoxifen induction of SMMHC-CreER; Rosa26-

Rainbow.

Movie S1. Three-dimensional architecture of a simple alveolus.

Movie S2. Three-dimensional architecture of an alveolar complex. 
Gillich et al., p. 44

\section{Figure Legends}

\section{Figure 1. Single-cell labeling reveals the cellular structure of alveoli in the mouse lung.}

(A, B) Simple alveolus. Confocal projection (A) and diagram (B) of a simple alveolus (dashed outline) with genetic labeling of single AT1 cell (green) in a Hopx-CreER; Rosa26-mTmG lung dosed with limiting tamoxifen $(0.3 \mathrm{mg}) 5$ days prior to analysis at 2 months of age and co-stained with GFP antibodies to visualize labeled cells and fluorescent hydrazide to label elastic fibers (white). Thick elastic fibers (solid arrowheads in A) form a ring at the alveolar entrance (dotted grey circles in B). Diagram also shows en face view (right) of same alveolus. See also Movie S1. Scale bars, $50 \mu \mathrm{m}$. (C, D) Alveolar complex. Confocal projection (C) and diagram (D) as above of an alveolar complex. Thin elastic fibers (open arrowheads in $\mathbf{C}$, dotted grey lines in $\mathbf{D}$ ) subdivide the alveolar complex into 3 units, which are numbered and outlined in green, blue, and red in D. See also Movie S2. Scale bars, $50 \mu \mathrm{m}$. (E to K) Confocal projections showing morphologies and arrangement of single alveolar cells [AT1 cell (E, green), AT2 cell (F, blue, arrowhead), capillary aerocyte (G, red), general capillary cell (H, pink), pericyte (I, purple), fibroblast (J, yellow), and myofibroblasts (K, labeled with the multicolor fluorescent reporter Rosa26-Rainbow)] in alveoli (dotted outlines in $\mathbf{E}$ to $\mathbf{G}$ ) and relative to elastic fibers (white, $\mathbf{E}, \mathbf{G}$ to $\mathbf{K})$ in a Hopx-CreER; Rosa26-mTmG lung (E) dosed and analyzed as above, a Shh-Cre; Rosa26-mTmG lung (F) immunostained for GFP at 2 months, a VE-cadherin-CreER; Rosa26Confetti lung ( $\mathbf{G}$ and $\mathbf{H}$ ) dosed with $1 \mathrm{mg}$ tamoxifen at 3 weeks and analyzed at 2 months by imaging endogenous RFP fluorescence, a Pdgfra-CreER; Rosa26-mTmG lung (I) dosed with 2 mg tamoxifen at 3 months and immunostained for GFP, a Gli1-CreER; Rosa26-mTmG lung (J) dosed with 4 mg tamoxifen at 2 months and immunostained for GFP, and a SMMHC-CreER; Rosa26-Rainbow $(\mathbf{K})$ lung dosed with $0.1 \mathrm{mg}$ tamoxifen at P5, immunostained at P10 with 
SMA-Cy5 antibodies (not shown) and analyzed by imaging endogenous Cerulean, mCherry and mOrange fluorescence. Scale bar, $50 \mu \mathrm{m}$. (L) Schematic of an alveolus with 7 major cell types (not showing immune cells) and a total of 12 cells: a single AT1 cell (green), an AT2 cell (blue), 3 capillary cells ( 1 aerocyte, red; 2 general capillary cells, pink; (53)), 2 pericytes (purple, with 1 on backside not shown), 1 fibroblast (yellow), and 4 myofibroblasts (orange) that associate with elastic fibers (brown) at the alveolar base. Grey ovals, nuclei.

\section{Figure 2. Mapping alveolar development at a specific position in branch lineage. (A) Branch}

lineage diagram for third anterior branch of left primary bronchus (L.L1.A3) showing descendant branches and position of the boundary (arrowhead) between conducting and respiratory airways along branches of bronchial generations 4-5. Airway generations are numbered. A, anterior; L, lateral; M; medial. (B to D) Confocal projections showing L.L1.A3 branch lineage in wild-type lungs immunostained for E-cadherin (epithelium, blue), SMA (smooth muscle, red) and Sox2 (conducting airways, green) at the indicated embryonic ages. The boundary between conducting and respiratory airways (solid arrowheads in C, D) is set on L.L1.A3 daughters by E16.5. Branches distal to the domain marked by Sox 2 (brackets in $\mathbf{C}, \mathbf{D}$ ) form respiratory airways; their names are indicated in top panels. SMA-positive cells (red) cover airway stalks and a few cells extend beyond the inferred future boundary position (open arrowheads in $\mathbf{B}$ ) at E15.5. Once the boundary is set, stalks of respiratory airways are surrounded by a layer of SMA-positive cells (brackets in $\mathbf{C}$ ). Note that the orientation of cells on airway stalks changes one day later (brackets in D). Scale bars, $50 \mu \mathrm{m}$. (E) Confocal projection with close-ups of boxed regions (E' to E'") showing SMA-positive cells (arrows in $\mathbf{E}$ ' to $\mathbf{E}$ ') on stalks of conducting $\left(\mathbf{E}^{\prime}\right)$ and respiratory airways (E') in an E16.5 SMA-CreER; Rosa26-tdTomato lung dosed with tamoxifen (2 mg) at E15.5 and immunostained for Sox2 (conducting airways, magenta), SMA (smooth muscle, red) 
and the lineage tag (tdTomato, pseudocolored in green). Note similar morphology and circumferential orientation of the cells at both positions at this stage. Arrowheads, compartment boundary. Scale bars, $50 \mu \mathrm{m}$. (F) Confocal projection with close-up of boxed region $\left(\mathbf{F}^{\prime}\right)$ as above in panel $\mathbf{E}$ showing SMA-positive cells (arrows in $\mathbf{F}^{\prime}$ ) on stalks of respiratory airways in an E17.5 SMA-CreER; Rosa26-tdTomato lung dosed with tamoxifen (2 mg) at E15.5. The lineage tag (tdTomato) is pseudocolored in green. Note that at this stage (E17.5) the cells have reoriented and form rings (asterisks in $\mathbf{F}^{\prime}$ ) on stalks of respiratory airways. Scale bars, $50 \mu \mathrm{m}$.

\section{Figure 3. Lineage tracing and clonal analysis show that airway smooth muscle cells become}

alveolar myofibroblasts. (A) Strategy of lineage trace of airway smooth muscle. Lgr6-CreER; Rosa26-tdTomato lungs were dosed at E14.5 with saturating (4 mg) tamoxifen to label smooth muscle on airway stalks with heritable tdTomato expression (red bar on diagram, with tamoxifen assumed active for 48 hours). Labeling (left panel of schematic) was analyzed at P10 and P60 once alveoli have formed (right panel of schematic). (B and $\mathbf{C}$ ) Confocal projections with closeups of boxed regions (B', C') showing alignment of lineage-labeled cells (arrows in B', C') with elastic fibers (blue) at the alveolar entrance in Lgr6-CreER; Rosa26-tdTomato lungs dosed as above and stained at P10 (B) or P60 (C) for the lineage tag (tdTomato, red), SMA (smooth muscle actin, green) and elastin (detected by hydrazide; blue). Asterisks in B' and C' denote alveolar lumens. Lineage-labeled alveolar cells express SMA at P10 (B'), but not at P60 (C'). Arrowheads, compartment boundary (identified by staining with fluorescent streptavidin to detect epithelial biotin; not shown). Brackets, respiratory airways with lineage-labeled cells. Lineage-labeled cells are located on 2 generations of respiratory airways, which corresponds to the number of airway generations surrounded by smooth muscle beyond the compartment boundary at E16.5 (see Fig. 2C). Incomplete labeling of alveolar entrance rings on these airways 
may be due to inefficient recombination in airway smooth muscle (see Fig. S9). Scale bars, 50 $\mu \mathrm{m}$. (D) Strategy of single cell (clonal) labeling of smooth muscle. SMMHC-CreER; Rosa26Rainbow lungs were dosed at E15 with limiting doses (0.05 mg) of tamoxifen to label single smooth muscle cells (bottom diagram, left) in one of 3 colors (Cerulean, mCherry, mOrange). Labeling was analyzed around airways at P10 (bottom diagram, right). Blue bar, labeling window (tamoxifen assumed active for 48 hours). Vascular smooth muscle clones were not analyzed. (E) Confocal projection (left) with close-ups of boxed regions (E', E'') and diagram (right) of a mixed clone (Cerulean) generated with SMMHC-CreER; Rosa26-Rainbow as described in (D) with labeled airway smooth muscle cells (E') and myofibroblasts (E' ') that are aligned with rings of elastic fibers (white) at the alveolar entrance. Asterisks denote alveolar lumens. Cells in the clone are numbered. Arrowheads, compartment boundary. Scale bars, 50 $\mu \mathrm{m}$. (F) Confocal projection (left) and diagram (right) of myofibroblast clone (mOrange) generated with SMMHC-CreER; Rosa26-Rainbow as above. Cells in the clone are numbered. Two myofibroblasts (orange) are aligned with elastic fiber rings (white) at alveolar openings. Note incomplete labeling of entrance rings. Scale bars, $50 \mu \mathrm{m}$.

\section{Figure 4. Anatomical and developmental diversity of myofibroblasts. (A) Confocal} projections with close-ups of boxed regions (A' and $\mathbf{A}^{\prime \prime}$ ) of a P10 C57BL/6 lung stained for SMA (smooth muscle actin, red) and elastin (detected by hydrazide, white). Filled arrowheads, alveolar entrance myofibroblasts aligned with thick elastic fibers; open arrowheads, partitioning myofibroblasts aligned with thin fibers. Scale bars, $10 \mu \mathrm{m}$. (B) Single-molecule in situ hybridization for tdTomato (white), Fgf18 (red) and Acta2 (SMA, green) in P10 Lgr6-CreER; Rosa26-tdTomato lung labeled at P8. Fgf18+tdTomato+ cells (Lgr6 lineage-derived entrance myofibroblasts) (B') comprise a subset of Acta2+ cells ( $21 \pm 4 \%$; mean \pm s.d.; $n=3$ lungs $)$. The 
majority $(98 \pm 1 \%)$ of entrance myofibroblasts (Lgr6 lineage-labeled cells) express Fgfl8 and Acta2. Partitioning myofibroblasts (B') also express Fgfl8 and Acta2. Scale bars, $10 \mu \mathrm{m}$. (C) Schematic of entrance myofibroblasts (red) in simple alveoli (left) and alveolar complex (right) and partitioning myofibroblasts (pink) that subdivide alveolar complex into two units.

\section{Figure 5. Alveolar buds are led by single AT2 cells through nascent myofibroblasts.}

(A) Confocal projection with close-ups of boxed regions (A', side view and A', en face view) showing that single cuboidal epithelial cells (dotted circles) are surrounded by partially flattened cells (open arrowheads) and a ring formed by smooth muscle cells (arrows) that are aligned with elastic fibers (green) in an E17.5 wild-type lung stained for SMA (smooth muscle actin, red), elastin (detected by hydrazide, green) and E-cadherin (epithelium, blue). Filled arrowheads, compartment boundary. Scale bars, $20 \mu \mathrm{m}$. (B) Confocal slice with close-up of boxed region (B') of an E17.5 wild-type lung immunostained for FGF receptor 2 (Fgfr2, progenitors and AT2 cells, red), podoplanin (Pdpn, progenitors and AT1 cells, green), and E-cadherin (epithelium, white) showing that alveolar epithelial buds are led by single AT2 cells (asterisks; note Fgfr2 restriction to single cuboidal epithelial cells, or occasionally, groups of 2-3 cuboidal cells, denoted by crosses). Single AT2 cells (dotted circle in B') are surrounded by nascent AT1 cells (open arrowheads in B'). Clustered cuboidal cells in distal tips are progenitors (Fgfr2+ Pdpn+ cells) that have not yet undergone differentiation into AT2 or AT1 cells. (C) Confocal projections of an E18.5 wild-type lung immunostained for Fgfr2 (red) and E-cadherin (epithelium, white) showing regular salt-and-pepper pattern of the alveolar epithelium. Nascent AT2 cells are surrounded by 2-4 partially flattened cells that do not express Fgfr2 (nascent AT1 cells, numbered). Scale bars, $20 \mu \mathrm{m}$. (D) Diagrams of nascent alveolar buds, shown as side (top) and en face views (bottom), led by a single AT2 cell (black) through the nascent myofibroblasts (red) that synthesize and 
align with elastic fibers (green). (E) Diagram of airway with leading single AT2 cells (black) surrounded by AT1 cells (light grey) and ring of myofibroblasts (red).

Figure 6. Effect of Notch signaling inhibition on alveolar epithelial patterning. (A) Single confocal slices showing nascent alveolar buds of an E17.5 CBF1-H2B-Venus lung immunostained for GFP to detect Venus (Notch nuclear reporter, green), Fgfr2 (red), and Ecadherin (epithelium, white). Nascent AT2 cells (dotted circles and solid arrowheads) express Fgfr2, but not Venus. Neighboring AT1 cells (dotted lines and open arrowheads) express nuclear Venus, but not Fgfr2. (B) Effect of pharmacological Notch inhibition by DBZ on alveolar epithelial patterning. DBZ (30 $\mu \mathrm{M}$ per kilogram body weight) or vehicle were injected daily in wild-type mice (E16-E18, panel C). Single confocal slices of vehicle (control) or DBZ-treated E18.5 lungs immunostained for SftpC (progenitors and AT2 cells, green), podoplanin (Pdpn, progenitors and AT1 cells, red) and E-cadherin (epithelium, white). (C) DBZ dosing regime. (D) Quantification of AT2 cell clustering in E18.5 vehicle or DBZ-treated lungs (mean \pm s.d.; n=500 cells scored per mouse; 6 mice per treatment group; Wilcoxon rank sum test, p-value=0.002 $(* * *))$. (E) Quantification of AT2 cell proliferation in E18.5 vehicle or DBZ-treated lungs (mean \pm s.d.; $\mathrm{n}=500$ cells scored per mouse; 3 mice per treatment group; Wilcoxon rank sum test, $\mathrm{p}$ value $=0.69$ (n.s., not significant) comparing \% Ki67+ AT2 cells). (F) Effect of Notch inhibition on fate selection of alveolar epithelial progenitors isolated from E16.5 wild-type lungs and cultured in Matrigel with Fgf7 and Notch inhibitor DAPT or Notch1 or Notch2 blocking antibodies as indicated. Single confocal slices of cultures on day 4 immunostained for SftpC (progenitors and AT2 cells, green) and RAGE (progenitors and AT1 cells, red) and counterstained with DAPI (nuclei, blue). (G) Quantification of alveolar cell fate (mean \pm s.d.; 
$\mathrm{n}=500$ cells scored in 3 biological replicates). Scale bars, $20 \mu \mathrm{m}$. (H) Schematic of Notchmediated lateral inhibition of AT2 fate following AT2 induction by Fgf signaling.

Figure 7. Model of alveolar patterning and formation by direct budding. Diagram of developing respiratory airway as side (top) and en face (bottom) views with epithelium (grey) and surrounding smooth muscle (red). Alveoli form on stalks by epithelial budding through the smooth muscle, led by single AT2 cells (black) that get selected by Fgf signaling and Notchmediated lateral inhibition. Single AT2 cells are surrounded by 2-4 nascent AT1 cells (light grey) that are specified by another signal (dotted arrows), possibly a mechanical signal (46). As the nascent AT2 cell buds, smooth muscle cells rearrange around it, forming a ring of 3-5 myofibroblasts that deposit elastin (asterisk) and define the alveolar entrance. Groups of budding cells (2-3 cells, see Fig. 5B) may be sites of alveolar complex formation (dashed arrow).

Outpocketing to form alveoli is accompanied by flattening and rounding of AT1 cells into a cup shape (one cell highlighted in green) and may be driven by mechanical forces around birth. 


\section{Supplementary Figure Legends}

Figure S1. Textbook model of alveolar development by septation. Following branching morphogenesis ('pseudoglandular' stage, mouse: E9.5-E16.5, human: 5-17 weeks), the epithelium in distal branch tips differentiates to form squamous AT1 cells (grey) and cuboidal AT2 cells (black) ('canalicular' stage, mouse: E16.5-E17.5, human: 17-24 weeks) and the airspaces widen to form thin-walled saccules ('saccular' stage, mouse: E17.5-P4, human: 24 weeks to birth). The terminal sacs (top panel) are subdivided in the postnatal mouse lung into alveoli (bottom panel) by ingrowth of ridges or crests ('secondary septa') ('alveolarization' stage, mouse: P4-P30 (25, 27); human: $29-33$ weeks to several years $(30,31,97))$. Septation is thought to be driven by contraction or inward migration of myofibroblasts (red) $(25-29,44,96)$.

Figure S2. Structure and types of alveolar elastic fibers. (A and B) Confocal slice (A) and projection (B) showing conducting and respiratory airways (A) and alveolar entrance rings (B) in a 2 month-old wild-type lung stained with fluorescent hydrazide to visualize elastic fibers (white). Arrowheads, boundary between conducting and respiratory airways (note the abrupt transition in mice) (57). Scale bars, $50 \mu \mathrm{m}$. (C) Confocal projection with close-ups of boxed regions ( $\mathbf{C}^{\prime}$ to $\mathbf{C}^{\prime \prime \prime}$ ) showing three types of alveolar elastic fibers (thick, thin and microvascular fibers; arrowheads in $\mathbf{C}^{\prime}$ to $\mathbf{C}^{\prime \prime \prime}$ ) in a 2 month-old lung stained with fluorescent hydrazide (green) and anti-elastin antibodies (red). Note correspondence between hydrazide and elastin antibody stains. Scale bars, $25 \mu \mathrm{m}$. (D and E) Diagrams showing the arrangement of elastic fibers in simple alveoli (D) and alveolar complexes (E). Thick fibers (green circles) form a ring at the alveolar entrance, microvascular fibers (red) are interwoven with capillaries (grey), and thin fibers (blue) subdivide a complex into individual units (the complex depicted in $\mathbf{E}$ is 
composed of 2 units). (F) Simple alveolus on late generation respiratory airway. Respiratory airway generations (left panel, dashed lines) are numbered (arrowheads, generation 1). Inset (right) shows close-up of boxed region highlighting a single, GFP-labeled AT1 cell (green) in this Hopx-CreER; Rosa26-mTmG lung dosed with limiting tamoxifen (0.3 mg) 5 days prior to analysis at 2 months of age and co-stained with GFP antibodies to visualize labeled AT1 cells and fluorescent hydrazide to label elastic fibers (white). (G) Quantification of the proportion (in $\%$ of alveoli) of simple alveoli and alveolar complexes in adult mouse lung ( $\mathrm{n}=3 \mathrm{C} 57 \mathrm{BL} / 6$ lungs at 3 months of age stained with hydrazide; see Methods).

\section{Figures S3. Morphology and arrangement of AT1 cells in simple alveoli. Confocal}

projections (top) and diagrams (bottom) showing the arrangement of single AT1 cells (green) in simple alveoli (class 1). Note pores of Kohn (membrane-lined 'pores' (channels) through AT1 cells (52) that connect the lumens of adjacent alveoli, white ovals in diagrams; the number of pores (0-9) per cell is indicated below projection images) and thick elastic fibers forming a ring at the alveolar entrance (dotted circles in diagrams). Hopx-CreER or Foxa2-CreER;

Rosa26-mTmG lungs were dosed with tamoxifen $(0.3 \mathrm{mg}) 5$ days prior to analysis and stained at 2 months of age with GFP antibodies and fluorescent hydrazide (elastic fibers, white). Scale bars, $50 \mu \mathrm{m}$.

Figure S4. Morphology and arrangement of complex AT1 cells. Confocal projections (top) and diagrams (bottom) as in Figure S3 showing the arrangement of single AT1 cells (green) as part of alveolar complexes (class 2). Note pores of Kohn (white ovals in diagrams; the number of pores is indicated below projection images), thick elastic fibers forming a ring at the entrance of the alveolar complex (thick dotted circles) and thin fibers that subdivide alveolar complexes (thin 
dotted lines originating and terminating on thick dotted circle). Some AT1 cells could not be assigned to class 1 or class 2 , since they were positioned between alveoli, and they may be part of simple alveoli and/or alveolar complexes (class 3). Scale bars, $50 \mu \mathrm{m}$. Bottom graphs show quantification of the number of AT1 (left) and AT2 cells (right) in simple alveoli and alveolar complexes. Data are shown as boxplots with individual data points plotted. The number of cells was scored from 3D renderings of confocal z-stacks as described in the Methods. AT1 cells scored in $n=10$ simple alveoli and $n=11$ alveolar complexes; AT2 cells scored in $n=10$ simple alveoli and $n=13$ alveolar complexes in adult mouse lung ( $n=3$ lungs at 3 months of age stained with hydrazide; see Methods). Red bar, median value.

Figure S5. Morphologies and features of alveolar cells. (A) Confocal slice of a single AT1 cell (green) in a Foxa2-CreER; Rosa26-Confetti lung dosed with $0.5 \mathrm{mg}$ tamoxifen and immunostained at 2 months for RFP (pseudocolored in green), the apical AT1 marker Pdpn (red) and the basal AT1 marker RAGE (white). Adult AT1 cells co-express Pdpn and RAGE and have an expansive, squamous shape. Arrowhead, AT1 cell nucleus. Scale bar, $10 \mu \mathrm{m}$. (B) Confocal projection of a single AT1 cell (green) with close-up of boxed region showing pores of Kohn (arrowheads) in a Hopx-CreER; Rosa26-mTmG lung dosed with $0.3 \mathrm{mg}$ tamoxifen and stained at 2 months for GFP (green) and E-cadherin (white). Pores are surrounded by circular junctions formed by AT1 cells from adjacent airways. Individual pores are also connected by junctions (presumably autocellular junctions formed by a single AT1 cell). Scale bars, $10 \mu \mathrm{m} .(\mathbf{C})$ Confocal projections of single AT2 cells (green) with 1-4 apical domains (red, arrowheads) in a Foxa2-CreER; Rosa26-mTmG lung dosed with $0.3 \mathrm{mg}$ tamoxifen and stained for GFP (green), Muc1 (apical domains, red), SftpC (surfactant, white) and DAPI (nuclei, blue) at 2 months of age. Scale bar, $10 \mu \mathrm{m}$. (D) Confocal projections of single myofibroblasts (orange) that are 
aligned with thick elastic fibers (white) in a postnatal day 10 SMMHC-CreER; Rosa26-Rainbow lung dosed with $0.1 \mathrm{mg}$ tamoxifen at P5 and stained with fluorescent hydrazide (elastin, white). Myofibroblasts have a simple spindle shape with 2 or 3 cell processes (arrowheads). Dotted lines, entrance to simple alveoli or complexes. Scale bar, $10 \mu \mathrm{m}$. (E) Confocal projection of pericyte (see also Fig. 1I) in a Pdgfra-CreER; Rosa26-mTmG lung dosed with $2 \mathrm{mg}$ tamoxifen at 3 months and immunostained for GFP (green), Pecam1 (red) and elastin (visualized by hydrazide, white). Scale bar, $10 \mu \mathrm{m}$. (F) Confocal projection of alveolar fibroblast (see also Fig. $1 \mathrm{~J})$ in a Gli1-CreER; Rosa26-mTmG lung dosed with $4 \mathrm{mg}$ tamoxifen at 2 months and immunostained for GFP (green), Pecam1 (red) and elastin (visualized by hydrazide, white). Scale bar, $10 \mu \mathrm{m}$. (G) Quantification of the number of cells in a simple alveolus. Data are shown as boxplots with individual data points plotted; $n=10$ cells of each type scored in adult mouse lungs ( $\mathrm{n}=3$ lungs at 3 months of age) with mosaic labeling and immunostaining against common antigens as described in the Methods. Red bar, median value.

Figure S6. Single cell labeling of alveolar cells. (A, B) Confocal projections of single aerocytes in Apelin-CreER; Rosa26-Confetti lungs (A) and single general capillary cells in Aplnr-CreER; Rosa26-Confetti lungs (B) dosed with tamoxifen (A, $0.5 \mathrm{mg} ; \mathbf{B}, 0.1 \mathrm{mg})$ at 2 months and immunostained for RFP (pseudocolored in green), Pecam1 (red) and elastin (visualized by hydrazide, blue) (see also Gillich et al. (51)). Scale bar, $10 \mu \mathrm{m}$. (C) Confocal projections of single pericytes in Notch3-CreER; Rosa26-Confetti lungs dosed with $0.5 \mathrm{mg}$ tamoxifen at 3 months and immunostained for RFP (pseudocolored in green), Pdgfrb (red) and elastin (visualized by hydrazide, blue). Scale bar, $10 \mu \mathrm{m}$. (D) Confocal projections of single alveolar fibroblasts in Pdgfra-CreER; Rosa26-Confetti lungs dosed with $0.5 \mathrm{mg}$ tamoxifen at 2 months 
and immunostained for RFP (pseudocolored in green), Integrin $\alpha 8$ (Itga8, red) and elastin (visualized by hydrazide, blue). Scale bar, $10 \mu \mathrm{m}$.

Figure S7. Mapping the boundary between conducting and respiratory airways. (A) Left lobe of a wild-type (C57BL/6NCrl) lung immunostained for SMA (smooth muscle, white) with boxed region showing L.L1.A3 branch and its daughters (see also lineage diagram in Fig. 2A). M, medial, L, lateral, A, anterior, P, posterior, V, ventral, D, dorsal. Scale bar, $500 \mu \mathrm{m}$. (B) Lineage diagrams (top) and confocal projections (bottom) showing the position of the boundary between conducting and respiratory airways (arrowheads) on L.L1.A3 daughter branches in wild-type lungs stained at 2 months of age with SMA antibodies (smooth muscle, green), fluorescent streptavidin to detect biotin expressed by airway epithelial cells (red) and fluorescent hydrazide (elastin, blue). Panels show four examples of boundary patterns observed along L.L1.A3: symmetric at generation 4 (B'), asymmetric at generation 4 on the lateral aspect of L.L1.A3 and at generation 5 on the medial side (B'), asymmetric at generation 5 on the lateral side and generation 4 on the medial side (B'"), and symmetric at generation 5 (B'",'). See quantification in panel D. Scale bar, $500 \mu \mathrm{m}$. (C) Confocal projections showing that the compartment boundary (arrowhead in green panel) visualized by Sox 2 expression (green) corresponds to the biotin expression domain (arrowhead in red panel) detected by fluorescent streptavidin (red) in a 2 month old wild-type lung stained with anti-Sox 2 antibodies and Alexa Fluor 568 streptavidin. Scale bar, $50 \mu \mathrm{m}$. (D) Table showing the percentage of wild-type (C57BL/6NCrl) lungs at the indicated embryonic and adult ages with the boundary patterns shown in B' to B',', Note that the percentage of lungs with pattern B' (symmetrical at generation 4) is similar at E16.5, E18.5, and P60 (20-22\%), but higher in E15.5 (42\%), indicating 
that the junction is dynamic until it is permanently established at around E16-E16.5, consistent with previous studies (57).

Figure S8. Nascent myofibroblasts align with elastic fibers on respiratory airways. Confocal projection with close-up of boxed region showing myofibroblasts (red) organized as rings (asterisks) and aligned with elastic fibers (white) on the stalk of a respiratory airway in an E17.5 wild-type lung stained for SMA (smooth muscle actin, red) and elastin (detected by hydrazide; white). Note that several myofibroblasts (arrowheads in red panel) and fibers (arrowheads in white panel) form a ring (yellow asterisk). Scale bars, $50 \mu \mathrm{m}$.

Figure S9. Strategy to label airway smooth muscle. (A) Diagram showing genetic strategy to label smooth muscle on embryonic airways. Lgr6-CreER; Rosa26-tdTomato lungs were dosed at E14.5 with saturating ( $4 \mathrm{mg}$ ) tamoxifen to label smooth muscle on airway stalks with heritable tdTomato expression (red bar on diagram) and the labeling was analyzed at E16.5. (B) Confocal projections of an E16.5 Lgr6-CreER; Rosa26-tdTomato lung stained for SMA (smooth muscle, green), the lineage tag (tdTomato, red) and E-cadherin (epithelium, blue) showing lineagelabeled cells (red) on airways (asterisks), but not vessels (arrowhead). Scale bar, $50 \mu \mathrm{m}$.

Figure S10. The Lgr6 lineage gives rise to alveolar entrance myofibroblasts. (A, C) Strategy of Lgr6-CreER lineage tracing. Lgr6-CreER; Rosa26-tdTomato lungs were dosed at E17.5 (A) or P8 (C) with saturating (4 mg) tamoxifen to label Lgr6-expressing cells with heritable tdTomato expression (red bar on diagram, tamoxifen activity window, with tamoxifen assumed active for 48 hours) and the labeling was analyzed at P10. (B, D) Confocal projections showing lineage labeling of 3-4 generations (B') and 5-6 generations (D'-D'',) of respiratory airways (numbered) in P10 Lgr6-CreER; Rosa26-tdTomato lungs dosed at E17.5 (B, B') or at P8 (D, D'-D'''). 
Arrowheads, bronchoalveolar duct junctions. Scale bars, 1 mm (B, D), 50 um (B', D'-D','). (D',') Lgr6 lineage-labeled cells are aligned with thick (filled arrowhead) but not thin (open arrowhead) elastic fibers. (E) Quantification of the efficiency of labeling using Lgr6-CreER; Rosa26-tdTomato (data shown as mean \pm s.d., $\mathrm{n}=200$ cells scored on L.L1.A3.M.M/L respiratory airways, $\mathrm{n}=3$ mice per group). (F) Single-molecule in situ hybridization for tdTomato (white), Eln (tropoelastin, red) and Acta2 (SMA, green) in P10 Lgr6-CreER; Rosa26-tdTomato lung labeled at P8. Lgr6 lineage-labeled cells (asterisks) express Eln transcripts. Dashed oval, vessel wall. Scale bars, $10 \mu \mathrm{m}$. (G) Dot plot showing expression of tropoelastin (Eln) and matrix molecules that associate with elastic fibers (Fbln1, fibulin-1; Dcn, decorin; Mfap2 and Mfap5, microfibril-associated glycoproteins) in entrance myofibroblasts (identified by expression of Lgr6, Fgf18, Aspn) and alveolar fibroblasts (Pdgfra, Lrat, Itga8), but not pericytes (Pdgfrb, Trpc6, Postn). The cell types were annotated in Tabula Muris Senis scRNAseq Smart-seq2 data for adult mouse lung (63). (H) Schematic of entrance myofibroblasts in simple (left) and complex (right) alveoli.

\section{Figure S11. Airway clones generated by tamoxifen induction of SMMHC-CreER; Rosa26-}

Rainbow. Confocal projections (left) and diagrams (right) of airway smooth muscle clones (left panel shows coherent clones, right panel dispersed clones) generated by injecting pregnant SMMHC-CreER; Rosa26-Rainbow females with a limiting dose (0.05 mg) of tamoxifen at E15 to induce rare recombination events. Endogenous Cerulean, mCherry, and mOrange fluorescence was analyzed on postnatal day 10 in vibratome sections stained with fluorescent hydrazide (elastin, white) and cleared with Cubic1. Clones with vascular labeling were not analyzed. Cells in each clone are numbered. See also Table S2 for a complete list of clones. Scale bars, $50 \mu \mathrm{m}$. 
Figure S12. Mixed and boundary clones generated by tamoxifen induction of SMMHC-

CreER; Rosa26-Rainbow. Confocal projections (left) and diagrams (right) of mixed clones spanning conducting and respiratory airways (top) or clones at the boundary between conducting and respiratory airways (bottom) on postnatal day 10 generated by injecting pregnant SMMHCCreER; Rosa26-Rainbow females with a limiting dose $(0.05 \mathrm{mg})$ of tamoxifen at E15 to induce rare recombination events. The clones are composed of elongated cells with circumferential orientation around airway stalks (airway smooth muscle) and spindle-shaped cells aligned with elastic fibers at the alveolar entrance (myofibroblasts). Cells in each single-color clone are numbered with close-ups of boxed regions at right showing split channels. See also Table S2 for a complete list of clones. Scale bars, $50 \mu \mathrm{m}$.

Figure S13. Myofibroblast clones generated by tamoxifen induction of SMMHC-CreER; Rosa26-Rainbow. Confocal projections (left) and diagrams (right) as in Fig. S12 showing pure myofibroblast clones on postnatal day 10 generated by injecting pregnant SMMHC-CreER; Rosa26-Rainbow females with a limiting dose $(0.05 \mathrm{mg})$ of tamoxifen at E15 to induce rare recombination events. The clones are composed of cells located exclusively on respiratory airways. Cells in each clone are numbered. Asterisks denote alveolar lumens. Note that individual alveolar entrance rings are incompletely labeled suggesting they do not arise by clonal proliferation. See also Table S2 for a complete list of clones. Scale bars, $50 \mu \mathrm{m}$.

Figure S14. Epithelial pattern and budding on airway stalks through the nascent myofibroblasts. Series of confocal sections (corresponding to the projection image shown in Fig. 5A) showing that single cuboidal epithelial cells (yellow asterisks), or small groups of 2-3 cells (yellow crosses), are selected and protrude through smooth muscle (red) on airway stalks of 
an E17.5 wild-type lung stained for SMA (smooth muscle actin, red) and E-cadherin (epithelium, white). Scale bar, $50 \mu \mathrm{m}$.

Figure S15. The budding single cuboidal epithelial cells are nascent AT2 cells. (A) Confocal projection of an E17.5 wild-type lung stained for SMA (smooth muscle actin, red), Muc1 (progenitors and AT2 cells, green), and E-cadherin (epithelium, blue) with close-up of boxed region showing that single cuboidal epithelial cells (dotted circles) express Muc1 (green), whereas neighboring cells (asterisks; partially flattened AT1 cells) are Muc1-negative/low. Scale bars, $20 \mu \mathrm{m}$. (B) Single confocal slice of an E17.5 wild-type lung stained for SftpC (progenitors and AT2 cells, green), Pdpn (progenitors and AT1 cells, red) and E-cadherin (epithelium, white). Close-up of boxed region shows that the single cuboidal epithelial cells (dotted circles) are nascent AT2 cells that express SftpC, but not Pdpn. Cuboidal cells in distal tips co-express SftpC and Pdpn. They are progenitors that have not yet undergone differentiation. Scale bars, $20 \mu \mathrm{m}$.

Figure S16. The Notch signaling pathway is activated in nascent AT1 cells. (A) Confocal projection of an E18.5 CBF1-H2B-Venus lung immunostained for GFP to detect nuclear Venus (green) and for E-cadherin (epithelium, white). Note Notch reporter activity (green) in nascent AT1 cells (open arrowheads) but not nascent AT2 cells (solid arrowheads). Scale bar, $20 \mu \mathrm{m}$. (B) Confocal optical section of an E16.5 CBF1-H2B-Venus (Notch reporter) lung immunostained for GFP to detect nuclear Venus (green), Pecam1 (blue), E-cadherin (epithelium, white) and Fgfr2 (red). Note Notch activity (green) in endothelial plexus (blue), but not epithelial progenitors (white) that express Fgfr2 (red). Scale bar, $20 \mu \mathrm{m}$. (C) Confocal optical section of an E18.5 CBF1-H2B-Venus lung immunostained for GFP to detect Notch reporter (nuclear Venus, green), the Notch-induced gene Hes1 (red), Pdpn (progenitors and AT1 cells, white), and DAPI (nuclei, 
blue). Note colocalization of Hes1 (red) with nuclear Venus (green) in nascent AT1 cells (open arrowheads). Scale bar, $20 \mu \mathrm{m}$. (D) Confocal optical slides of an E18.5 wild-type lung immunostained for Hes1 (red), RAGE (AT1 cells, top panel, white) or E-cadherin (epithelium, bottom panel, white), Pdpn (green; progenitor and AT1 marker with apical localization), and DAPI (nuclei, blue). Note Hes1 localizes to nuclei of nascent AT1 cells (open arrowheads), but not AT2 cells (solid arrowheads). Scale bars, $20 \mu \mathrm{m}$. (E) Analysis of AT2 cell proliferation in embryonic lungs treated with Notch inhibitor DBZ. Confocal projection images showing alveolar epithelium of vehicle (control, top) or DBZ-treated (bottom) E18.5 lungs immunostained for SftpC (progenitors and AT2 cells, green), proliferation marker Ki67 (red) and DAPI (blue). DBZ (30 $\mu \mathrm{M}$ per kilogram body weight) or vehicle were injected daily in wild-type mice (E16-E18). See Fig. 6E for quantification of proliferation. Scale bars, $50 \mu \mathrm{m}$.

\section{Figure S17. Expression of Notch receptors and ligands in developing alveolar epithelial,}

endothelial, and stromal cells. (A) Heatmap showing normalized expression levels in individual alveolar epithelial cells from E18.5 mouse lung (scRNAseq data from Treutlein et al. (47)) for Notch receptors (green), ligands (red), and effectors. FPKM, fragments per kilobase of transcript per million mapped reads; Sftpc, progenitor and AT2 marker; Ager (RAGE), progenitor and AT1 marker; Cdhl (E-cadherin), epithelial marker; Gapdh, ubiquitous control. (B) Heatmap showing normalized expression levels in individual alveolar epithelial, endothelial, and stromal cells from developing mouse lung (E16.5 for epithelium, endothelium, and stroma; E18.5 for epithelium; scRNAseq data from Cohen et al. (74)) for Notch receptors (green), ligands (red), and effectors. UP10K, unique molecular identifiers per ten thousand. (C) Diagram summarizing expression of Notch receptors and showing potential cellular sources of the Notch signal in nascent alveolar buds. Multiple Notch receptors (green), canonical ligands (red), and 
non-canonical ligands (dark red) are expressed by developing alveolar epithelial cells (progenitors, AT2 and AT1 cells). The phenotype observed upon Notch inhibition in cultured alveolar epithelial progenitors (see Fig. 6F) suggests lateral inhibition of AT2 fate. The expression of $D l k 1$ in nascent AT2 cells is also consistent with a potential cis-inhibitory activity of Dlk1 to repress Notch signaling in AT2 cells (Dlk1 lacks the critical Notch binding (DSL) domain) (98). Alveolar endothelial and stromal cells are additional potential sources of a Notch signal. 

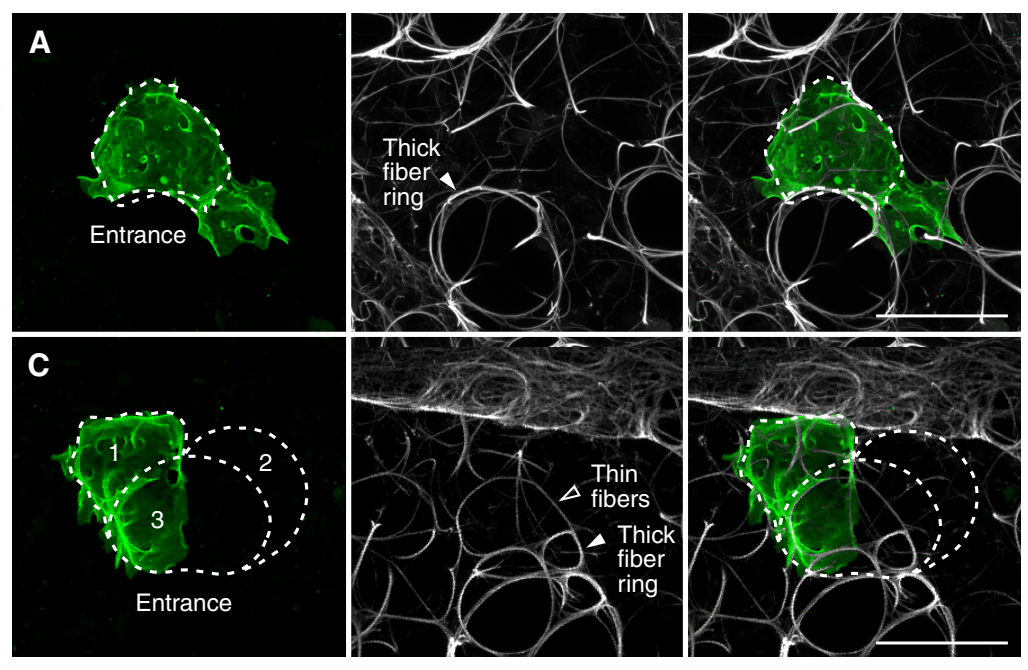

B

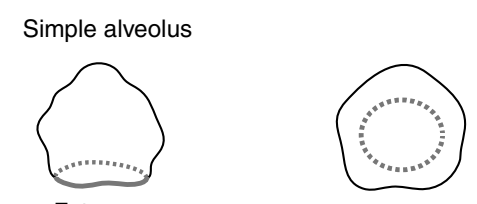

Entrance

Side

En face

D Alveolar complex
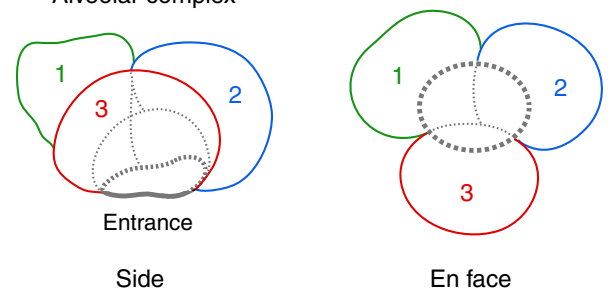

En face
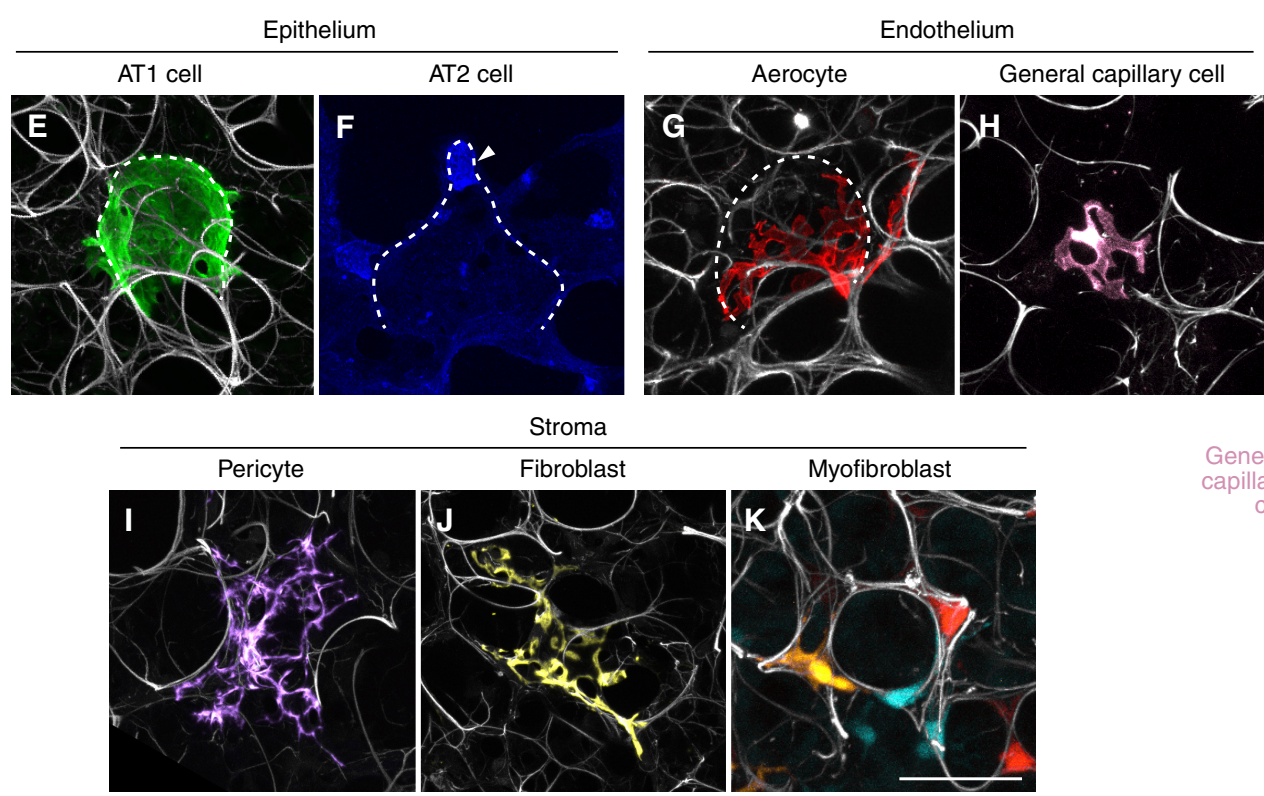

Stroma
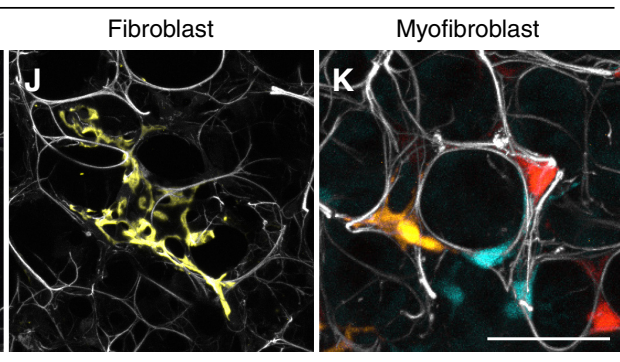

\section{L}

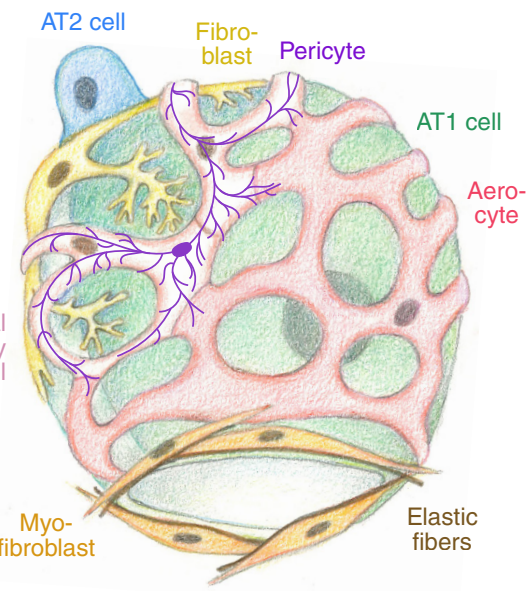



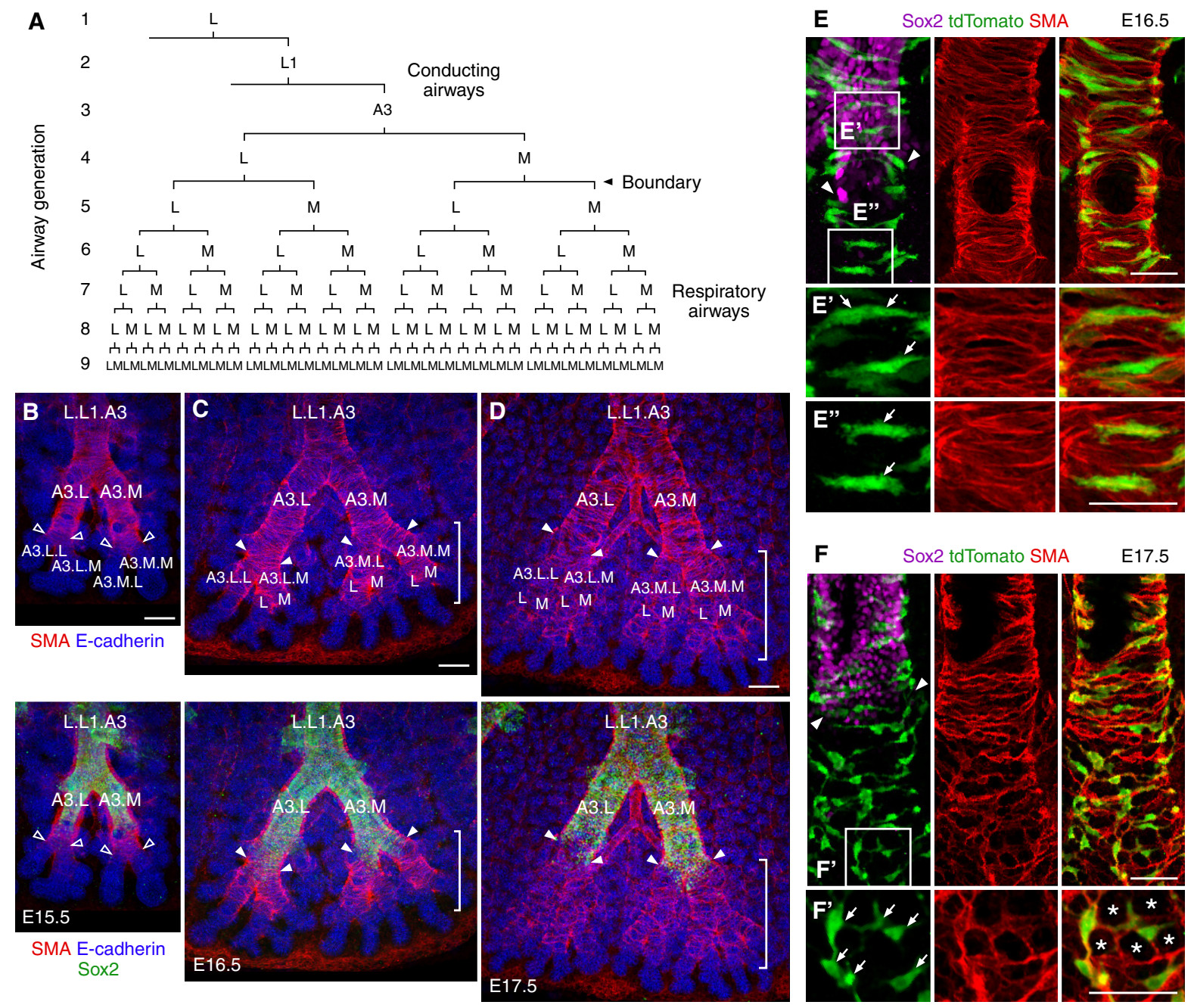
Gillich et al., Figure 3

A Labeling of airway smooth muscle (Lgr6-CreER; Rosa26-tdTomato)

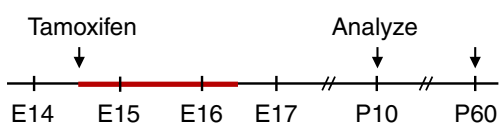

Lgr6-CreER; Rosa26-tdTomato E14.5 > P10
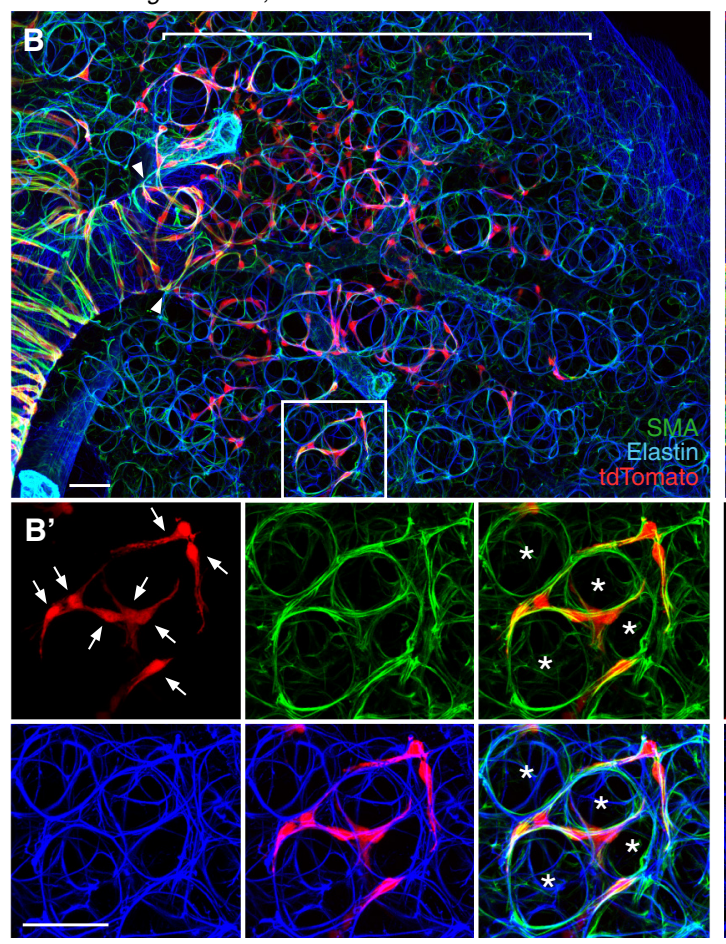

D

Single cell labeling of smooth muscle (SMMHC-CreER; Rosa26-Rainbow)
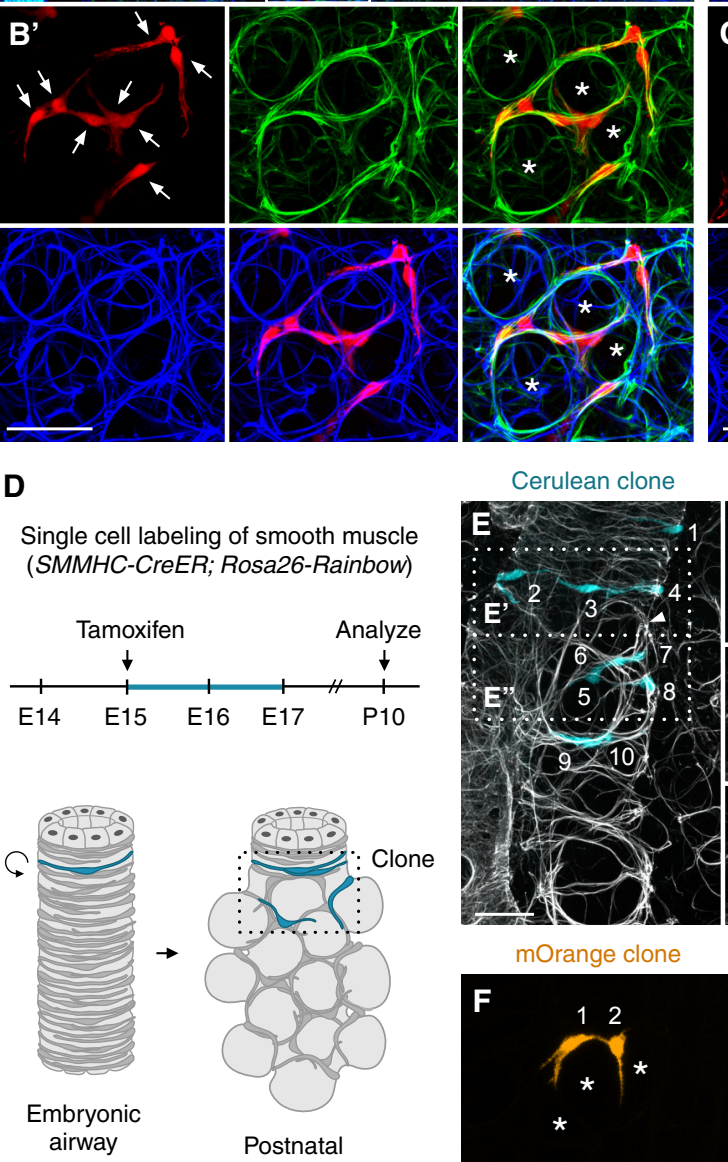

mOrange clone

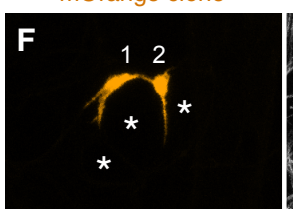

Airway smooth muscle
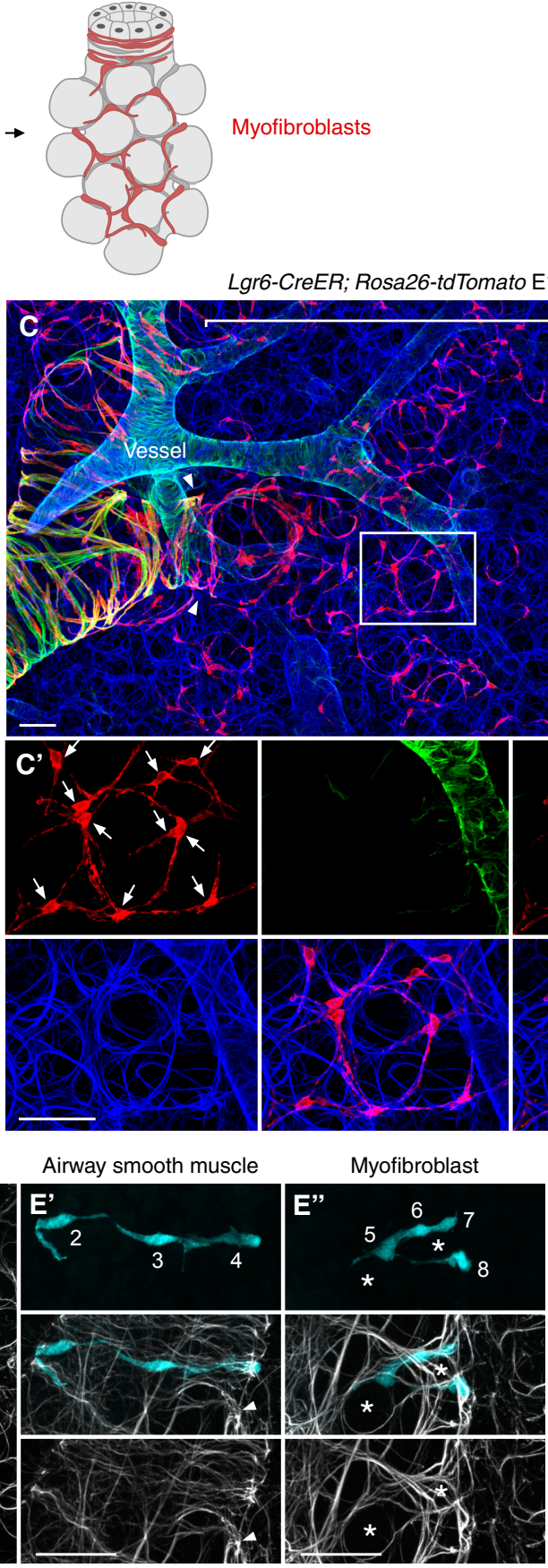

Lgr6-CreER; Rosa26-tdTomato E14.5 > P60

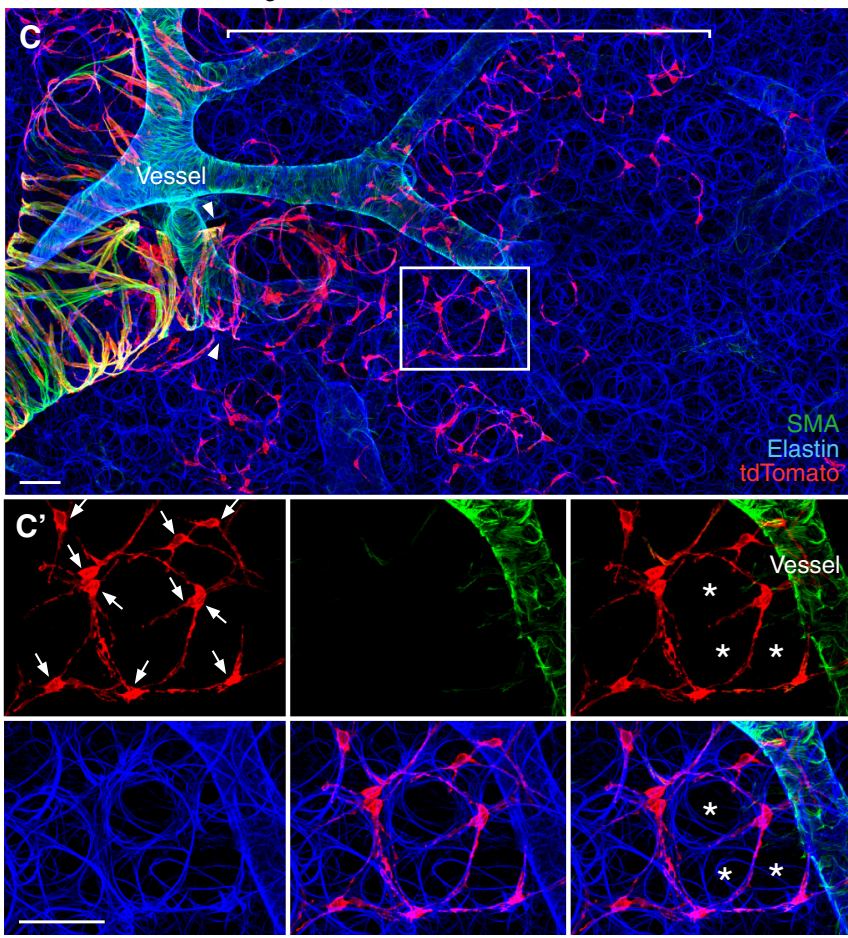

Myofibroblast

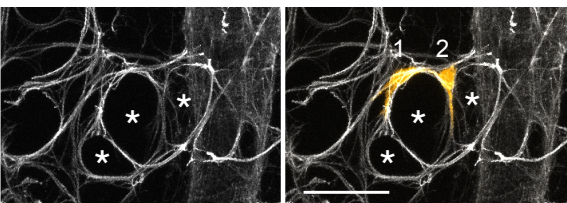

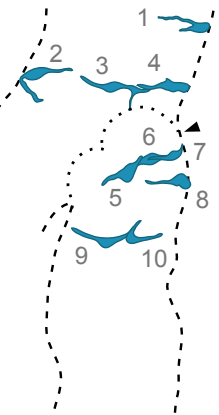

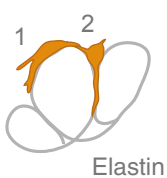




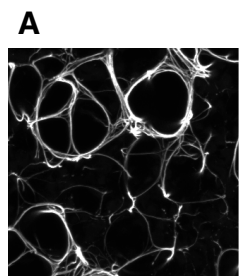

P10 lung

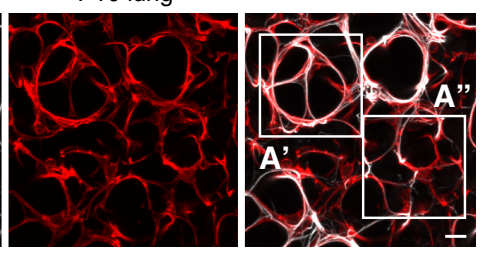

A'

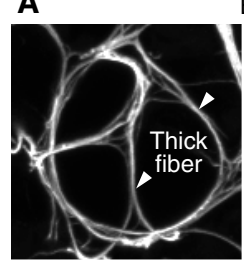

Entrance myofibroblast
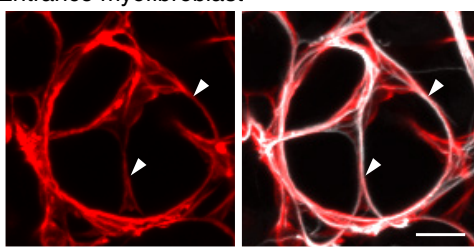

A" Partitioning myofibroblast
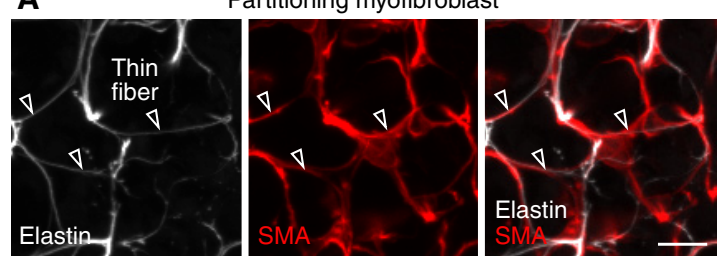

B

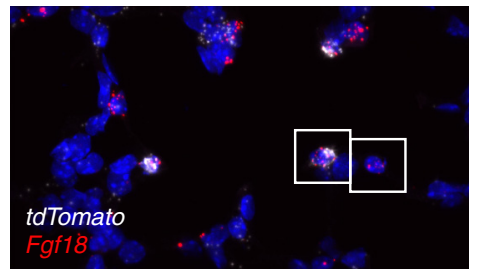

B'

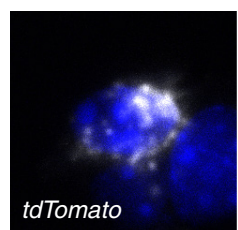

B"

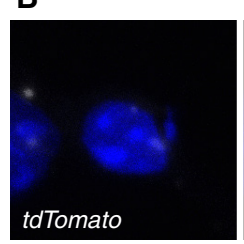

Gillich et al., Figure 4

C

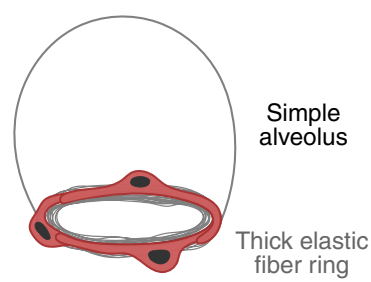

Entrance myofibroblasts Fgf18+ Lgr6 lineage ${ }^{+}$
Partitioning

myofibroblasts

Fgf18+

Lgr6 lineage-

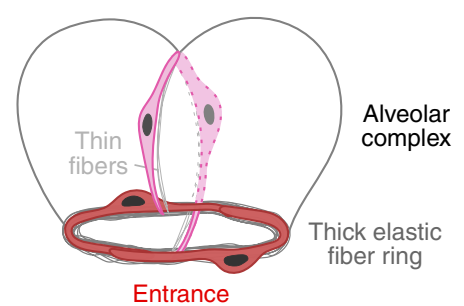

Entrance
myofibroblasts

$$
\text { Fgf18+ }
$$

Lgr6 lineage ${ }^{+}$ 


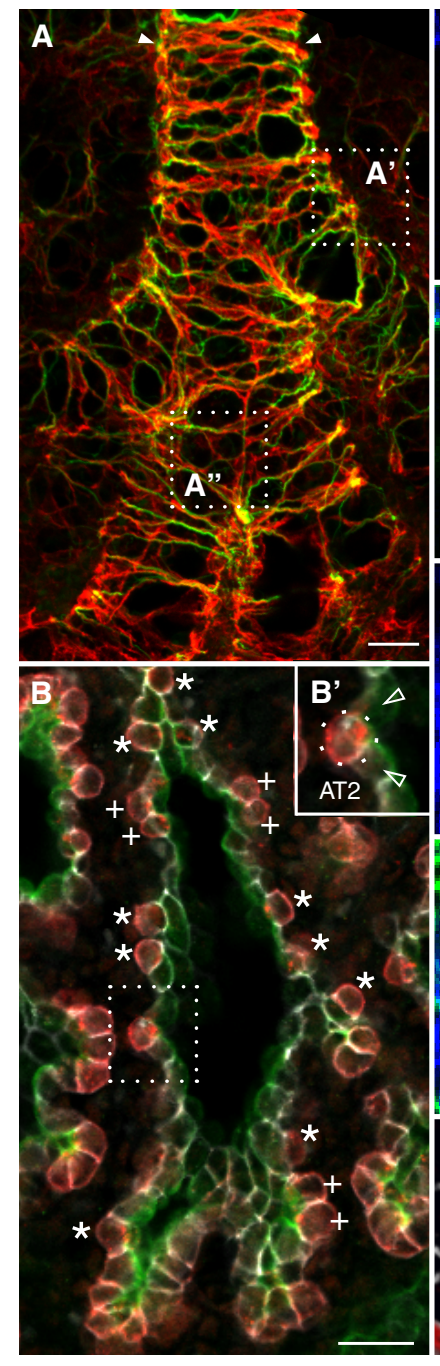

Fgfr2 Pdpn E-cadherin
SMA Elastin E-cadherin

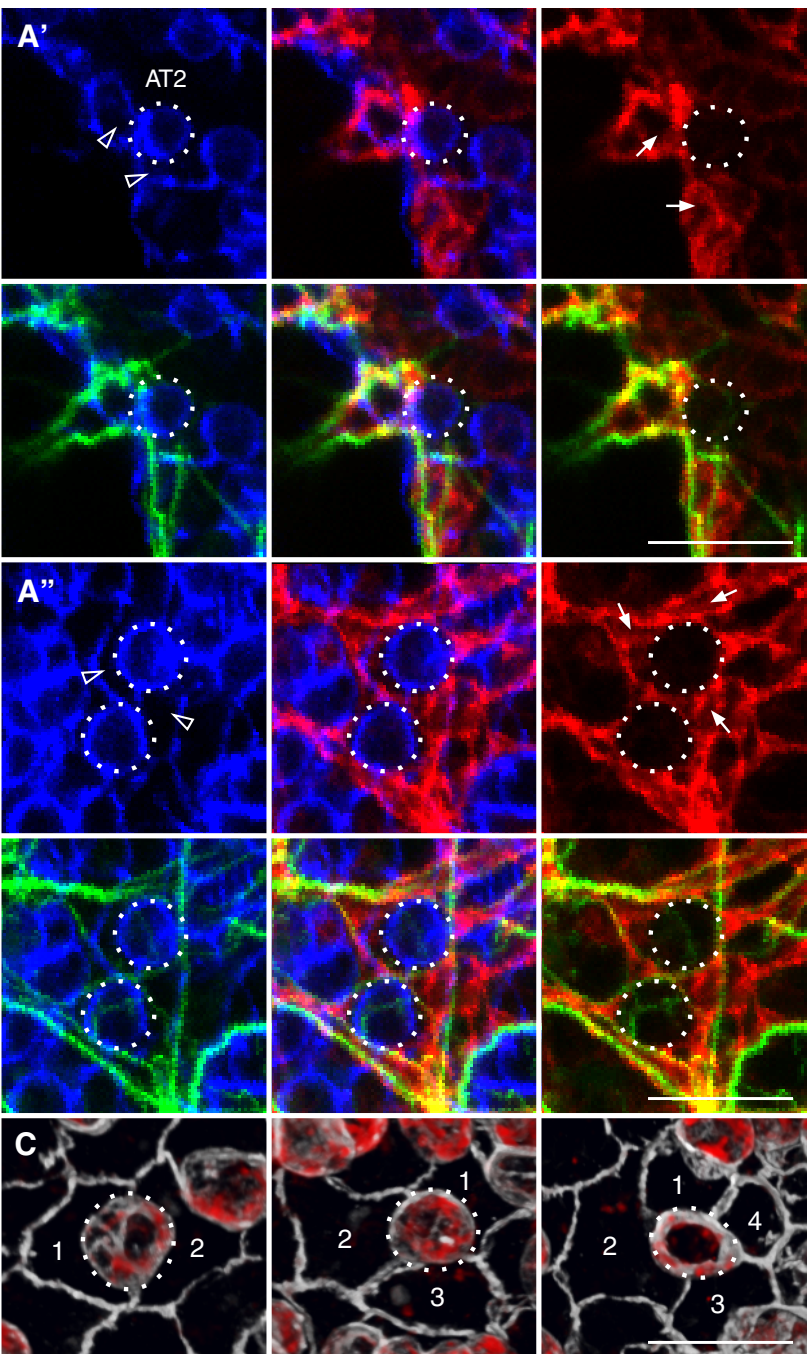

D

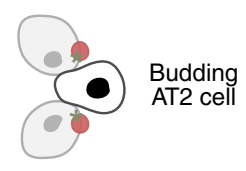

Side

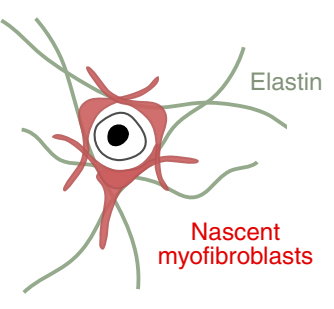

En face

E

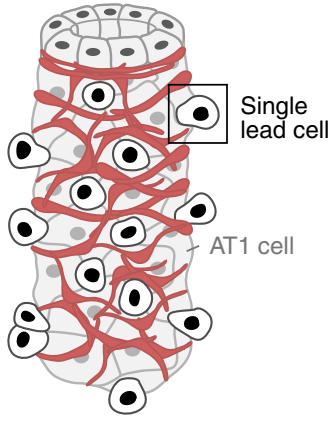

Embryonic

airway 


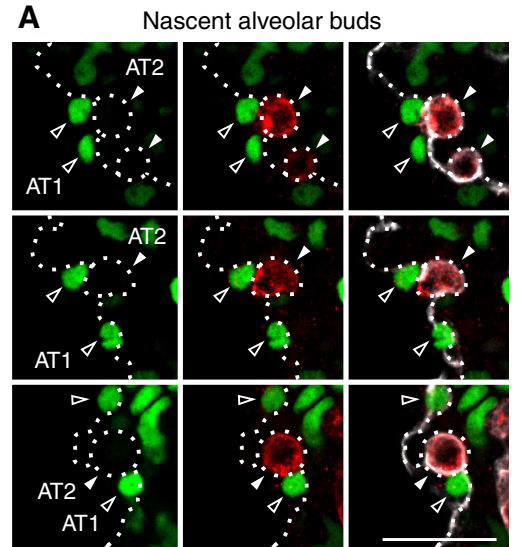

Notch reporter Fgfr2 E-cadherin
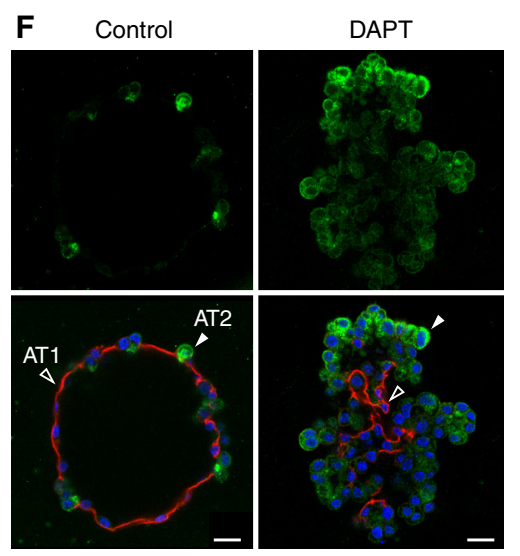

SftpC RAGE DAPI

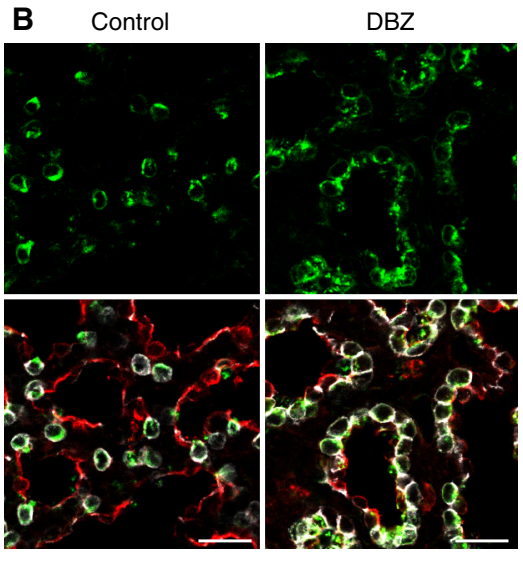

SftpC Pdpn E-cadherin

G

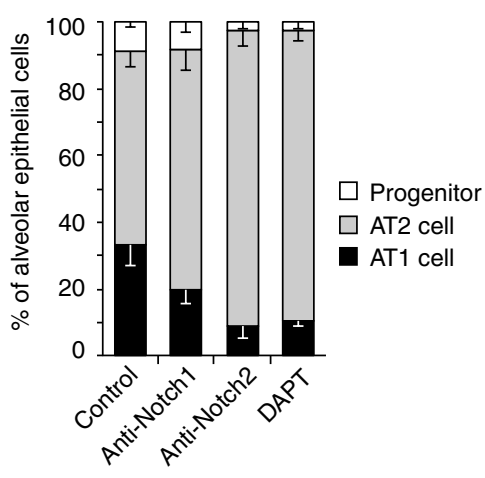

C
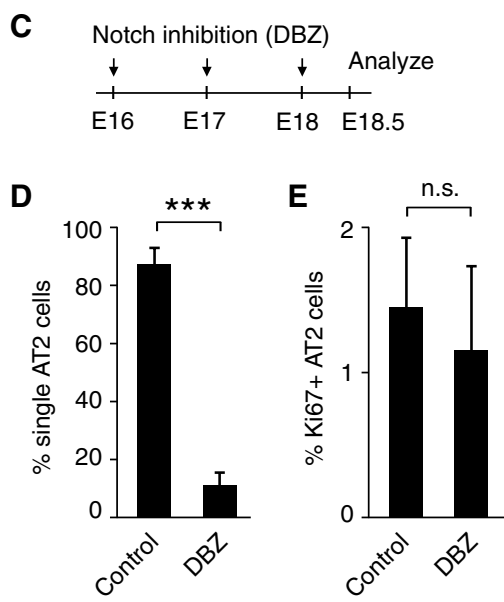

H

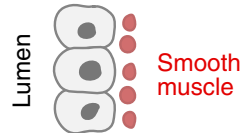

Epithelial progenitors

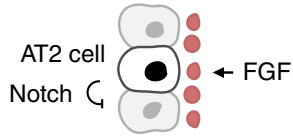

AT2 cell selection and lateral inhibition 
bioRxiv preprint doi: https://doi.org/10.1101/2021.12.25.474174; this version posted December 26, 2021. The copyright holder for this preprint (which was not certified by peer review) is the author/funder. All rights reserved. No reuse allowed without permission.

Gillich et al., Figure 7
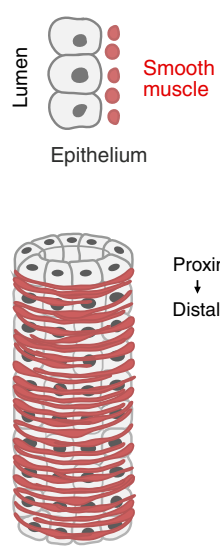

Proximal Distal

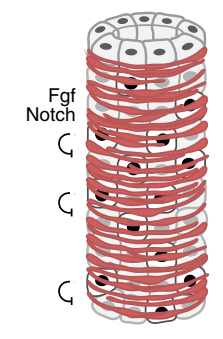

Embryonic airway

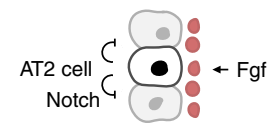

AT2 cell selection and lateral inhibition
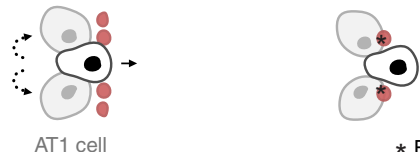

* Elastin

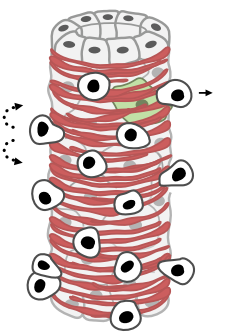

Budding of AT2 cell

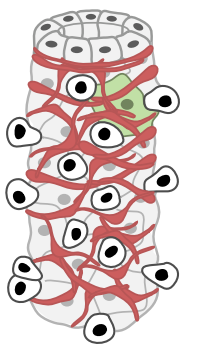

Smooth muscle

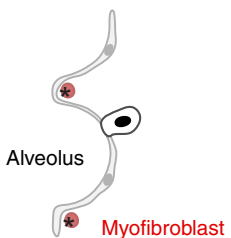
rearrangement to form myofibroblasts

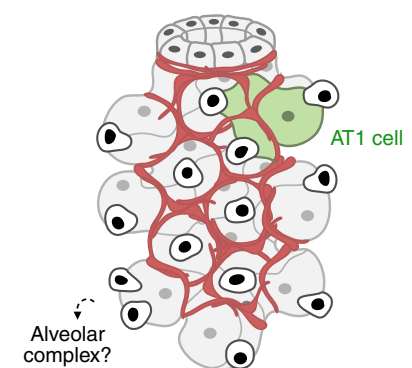

Outpocketing to form alveoli 
bioRxiv preprint doi: https://doi.org/10.1101/2021.12.25.474174; this version posted December 26, 2021. The copyright holder for this preprint (which was not certified by peer review) is the author/funder. All rights reserved. No reuse allowed without permission.

\section{Gillich et al., Figure S1}

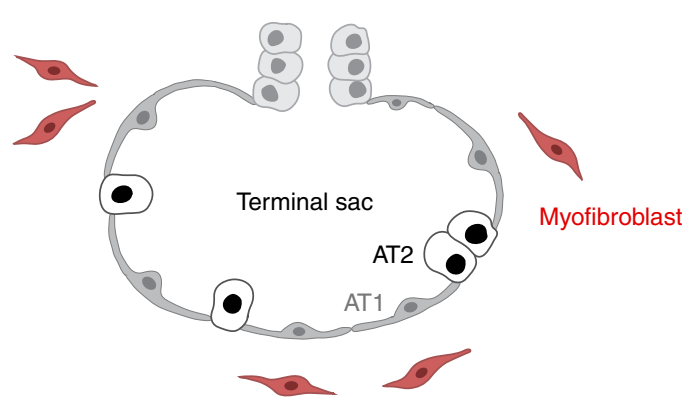

$$
\downarrow \text { Septation }
$$

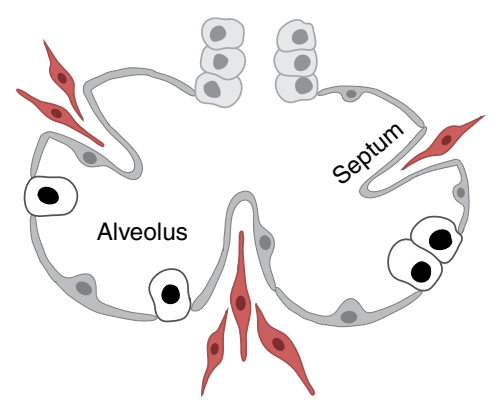




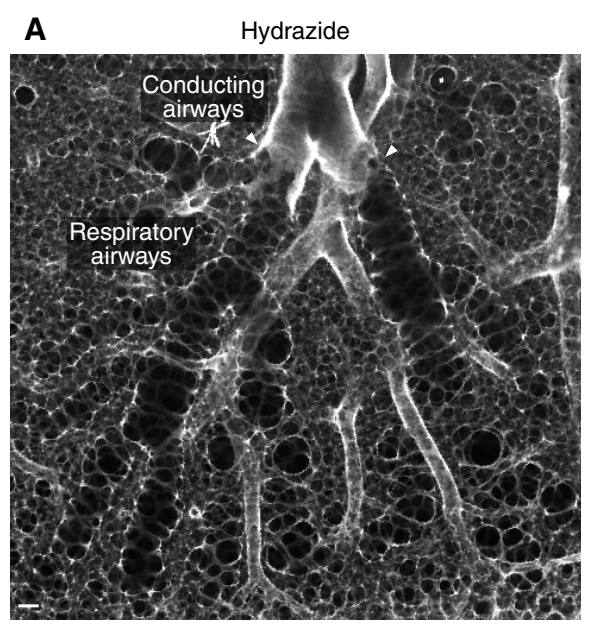

\section{B}

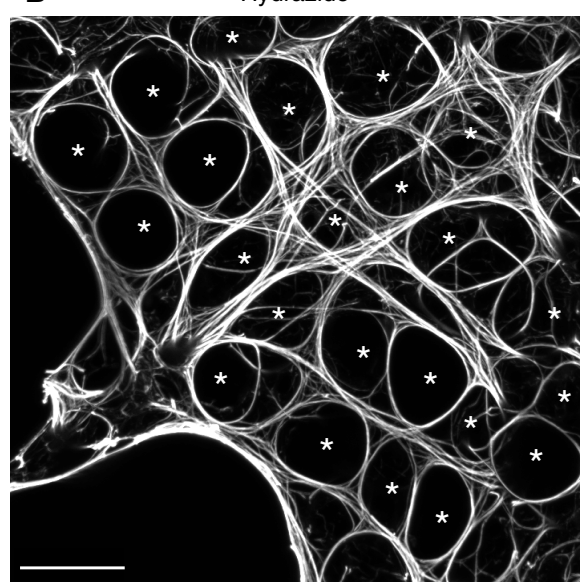

C

Hydrazide anti-elastin antibody

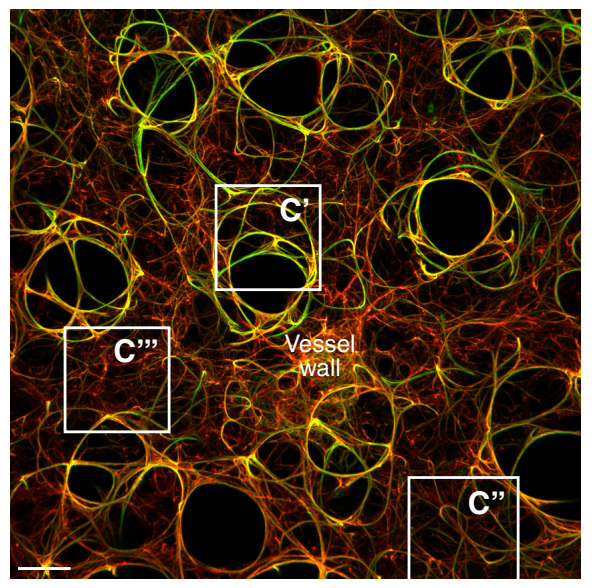

Hydrazide
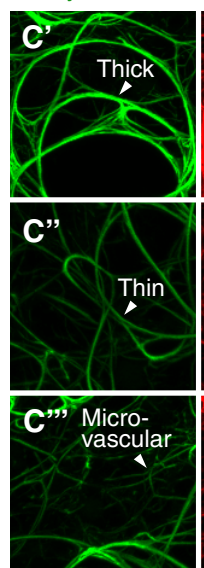

Anti-elastin antibody

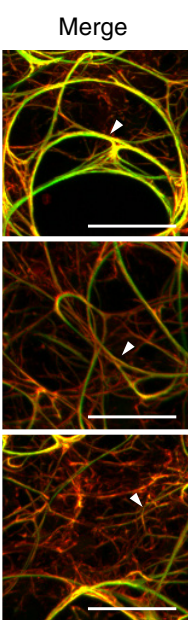

D

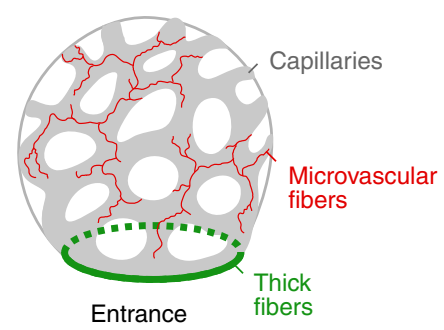

Simple alveolus

\section{E}

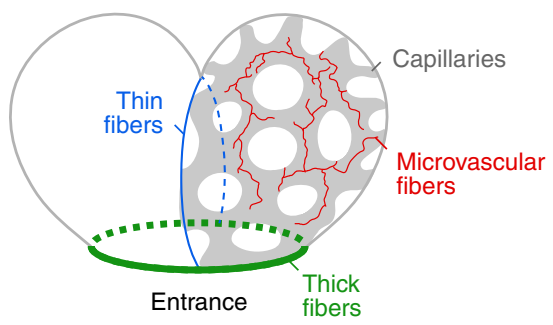

Alveolar complex
$\mathbf{F}$

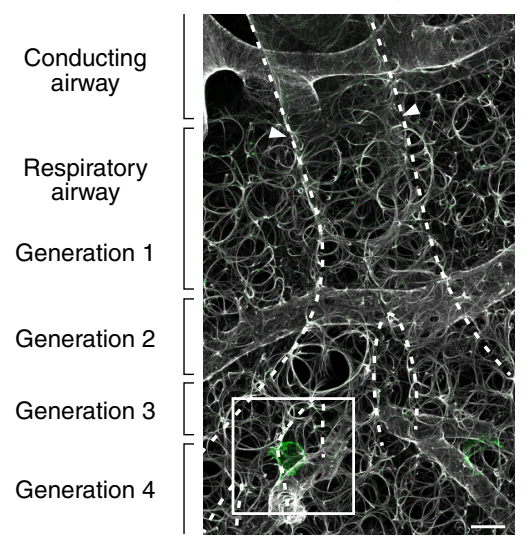

Hopx-CreER; Rosa26-mTmG

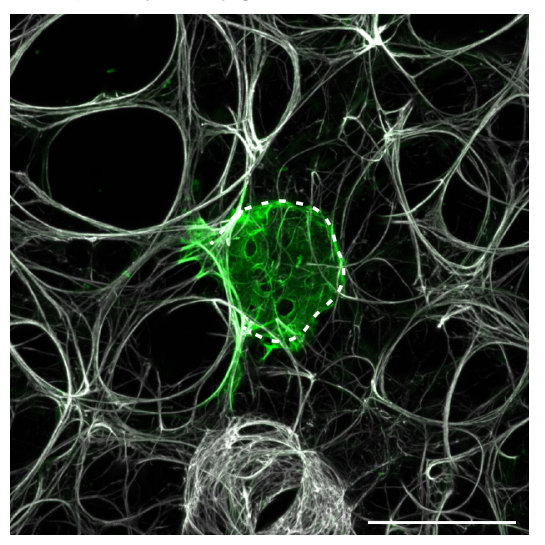

GFP Elastin (hydrazide)
G

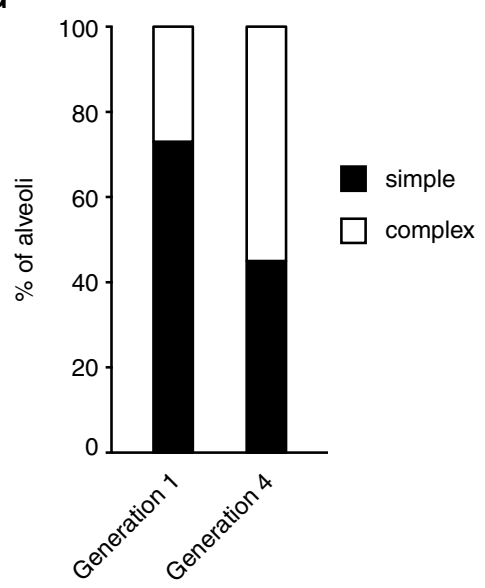


Class 1 (AT1 cell in simple alveolus)
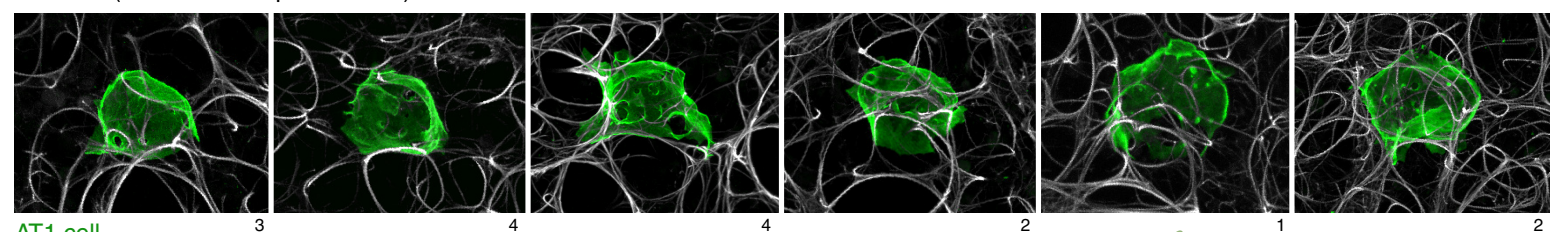

AT1 ce
Elastin
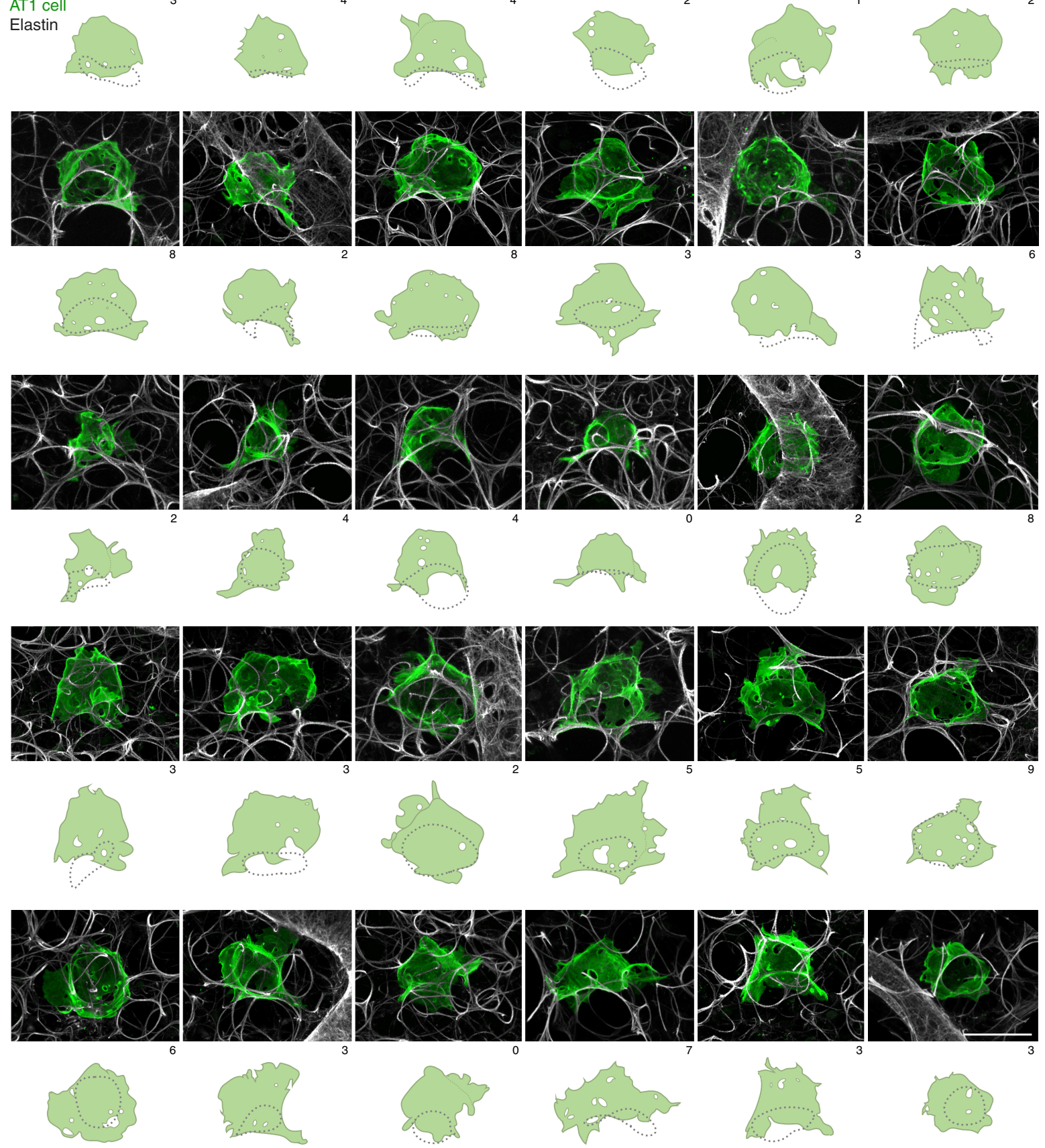
Class 2 (AT1 cell in alveolar complex)
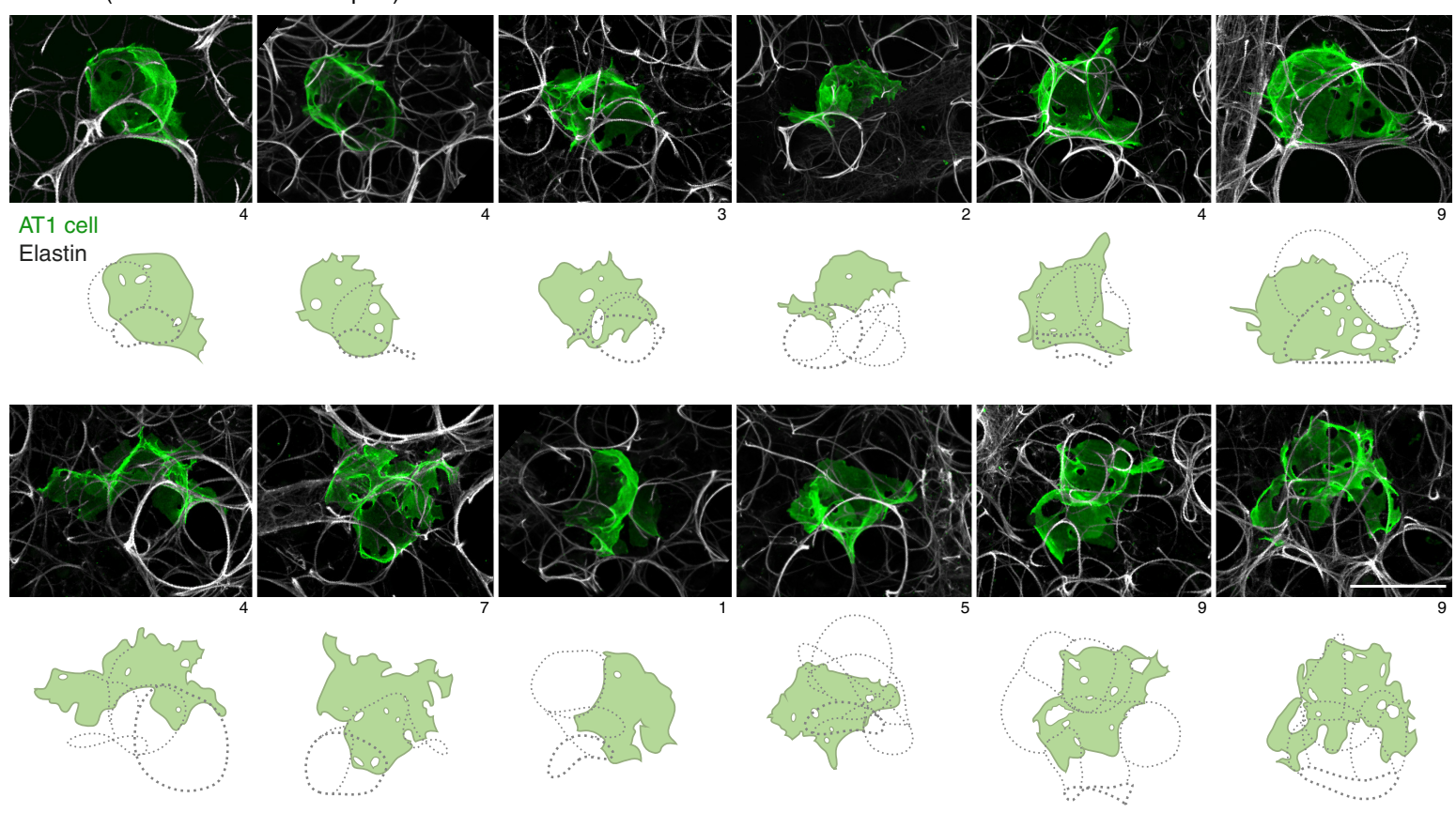

Class 3 (AT1 cell spanning simple alveoli and/or complexes)
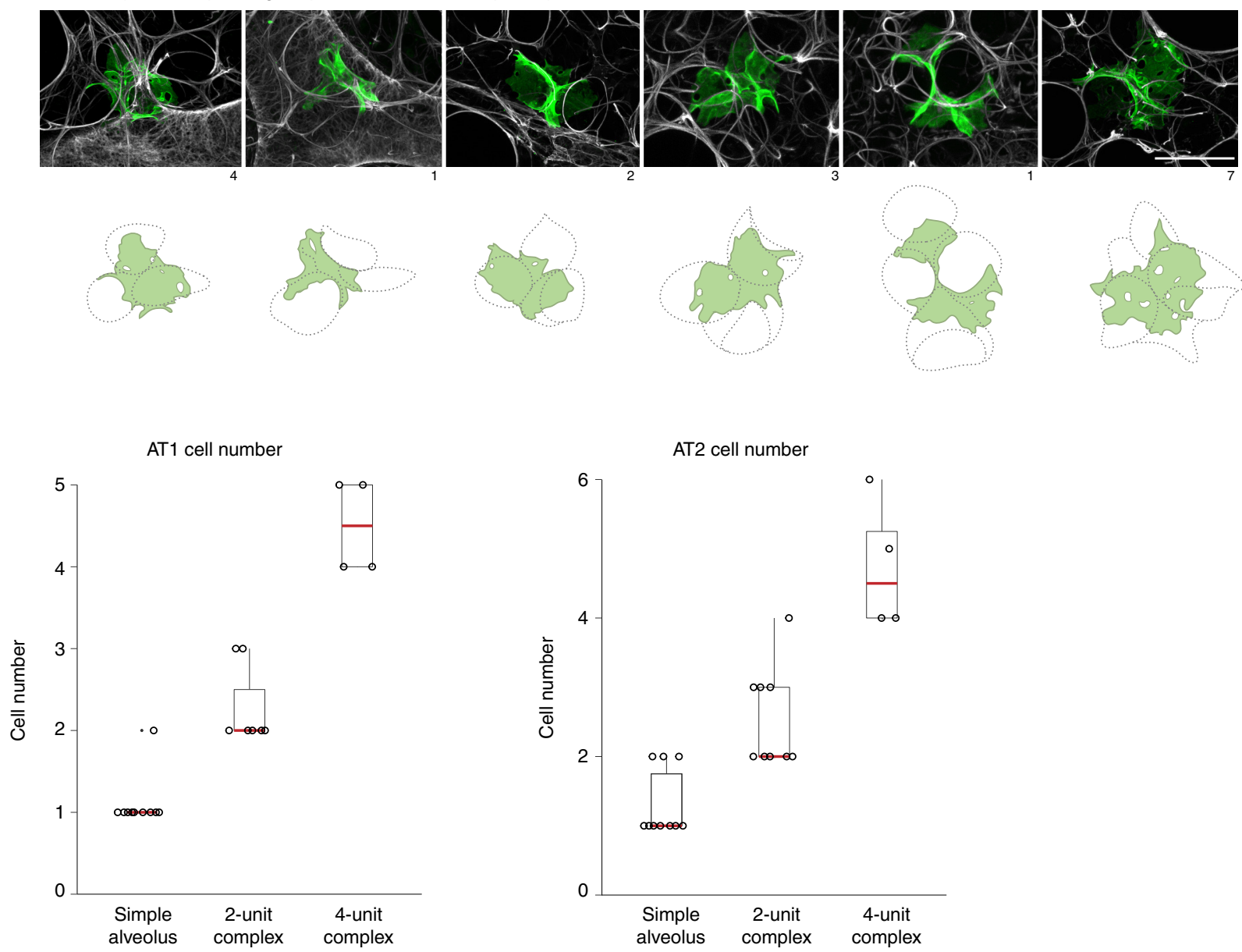

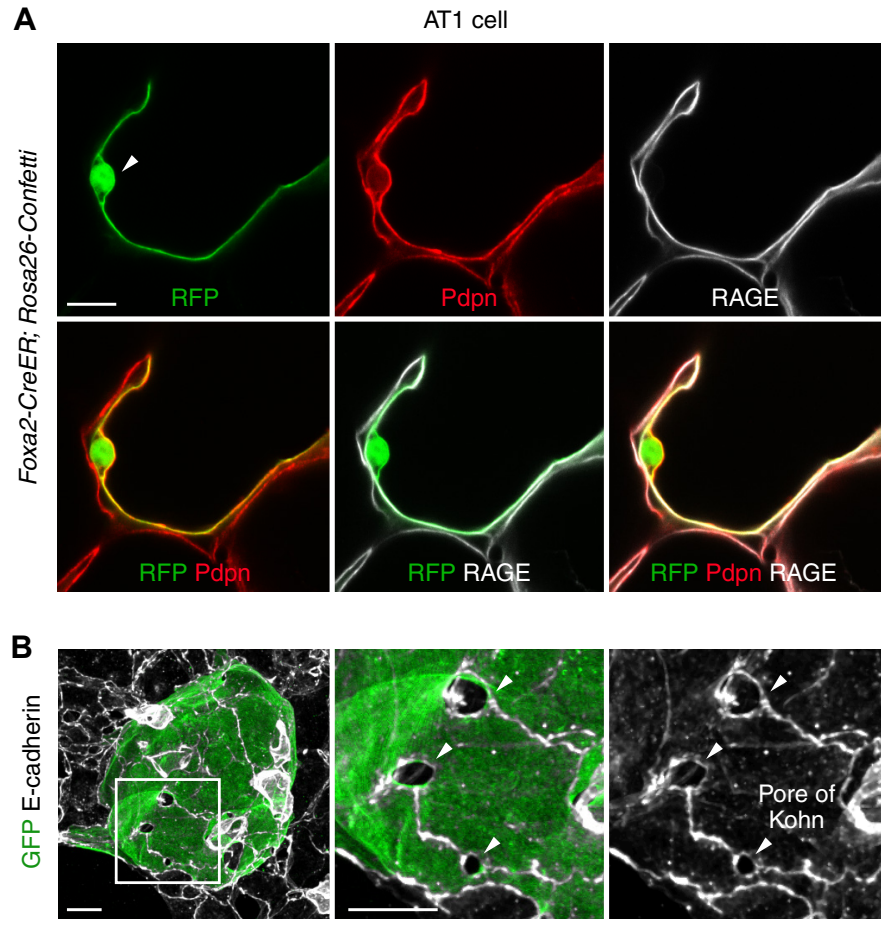

C

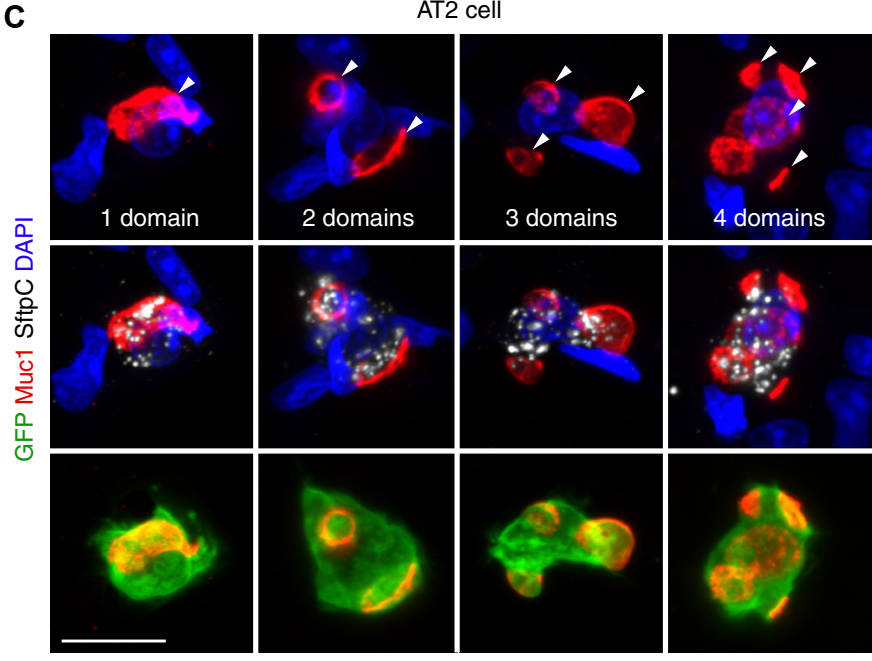

D

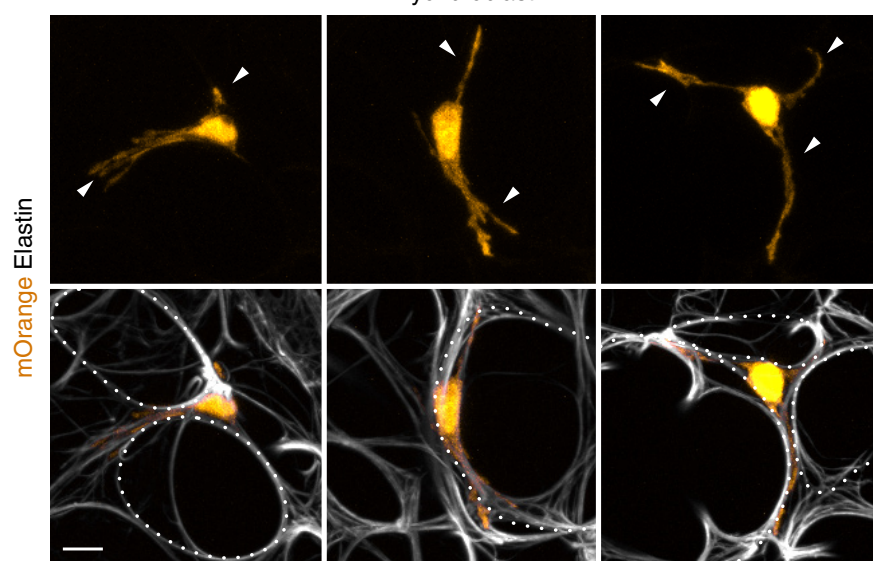

E

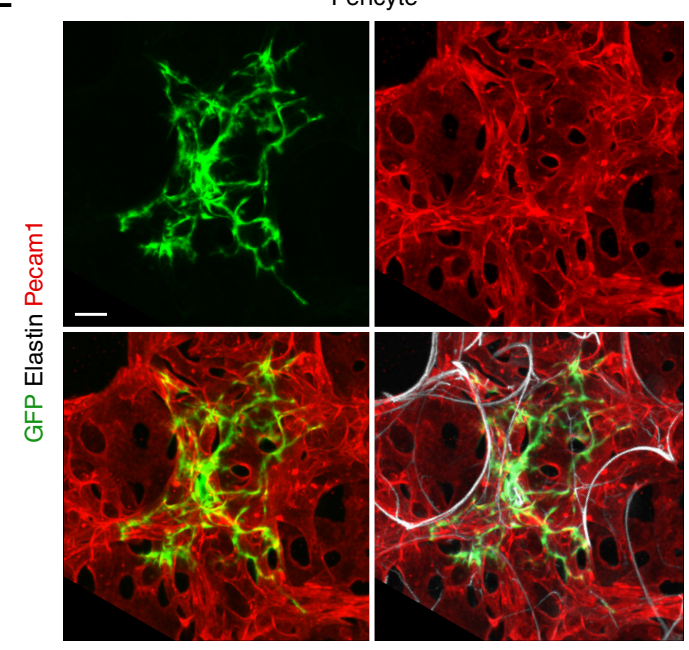

$\mathbf{F}$
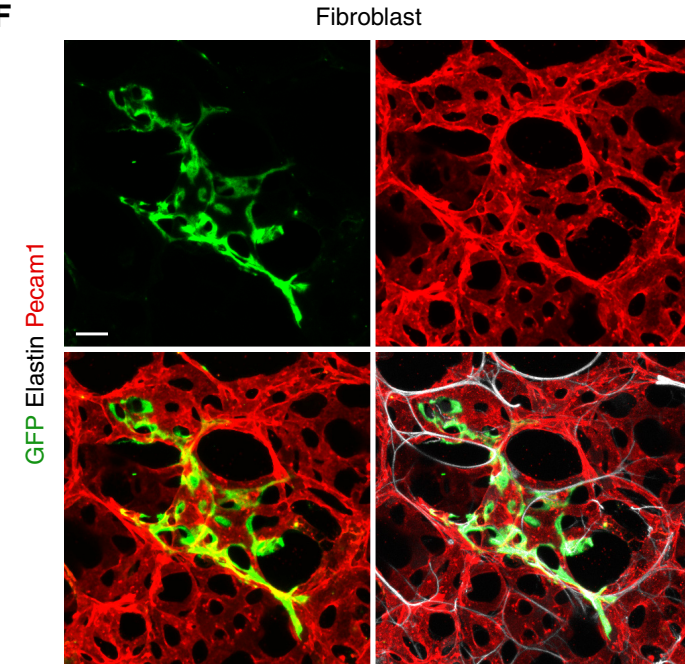

G

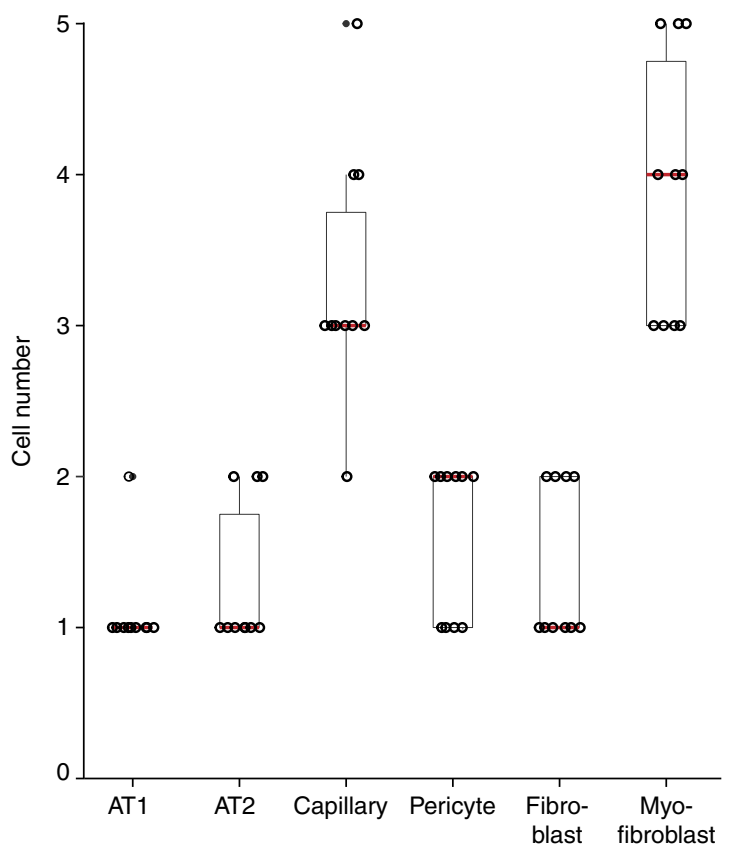


A

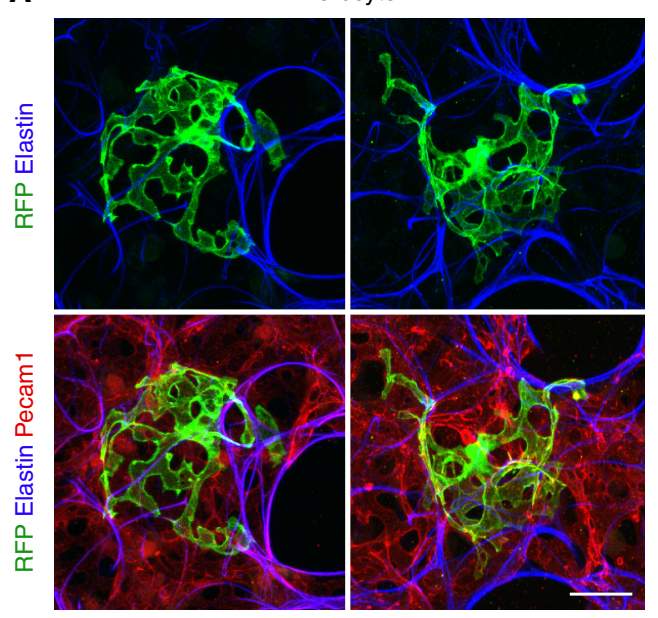

B

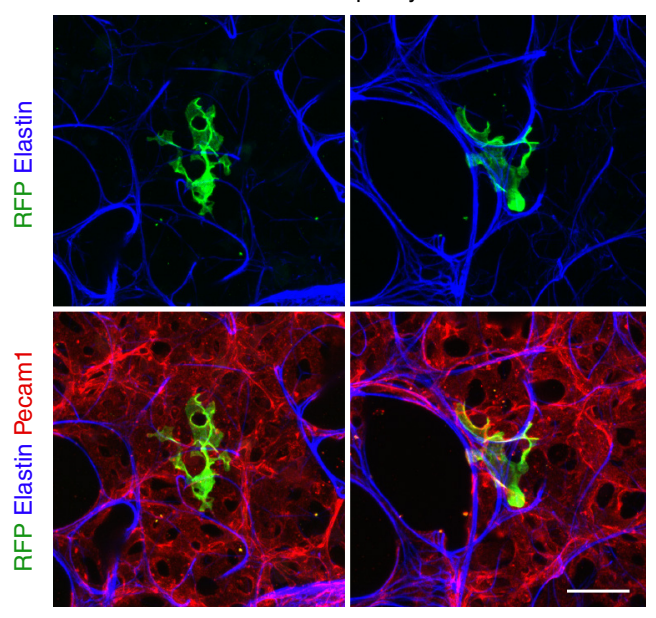

C

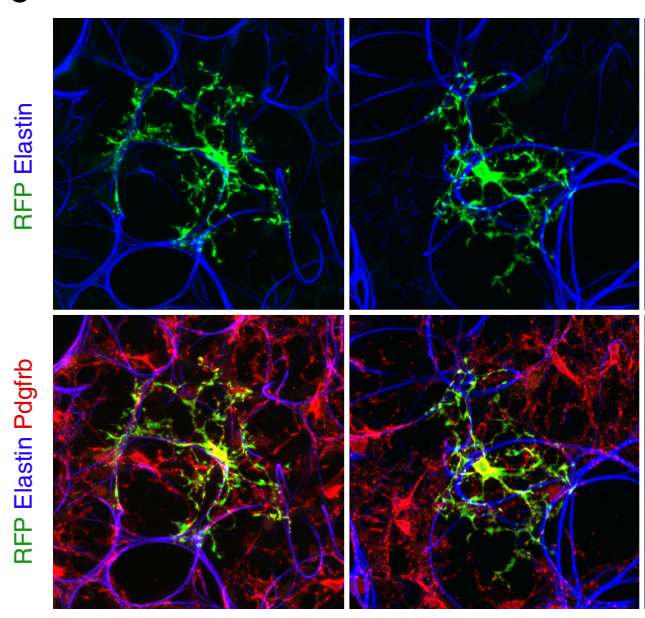

Alveolar pericyte

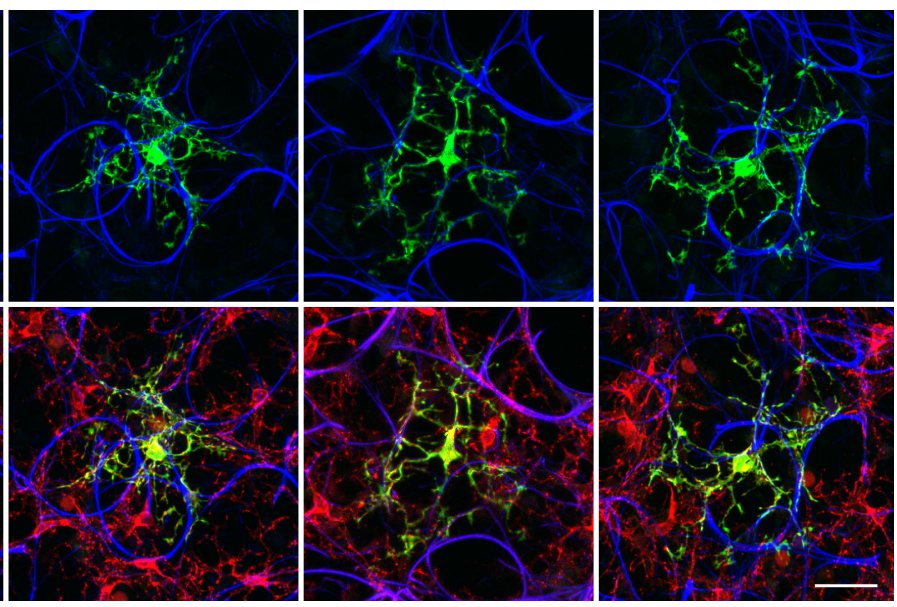

Alveolar fibroblast

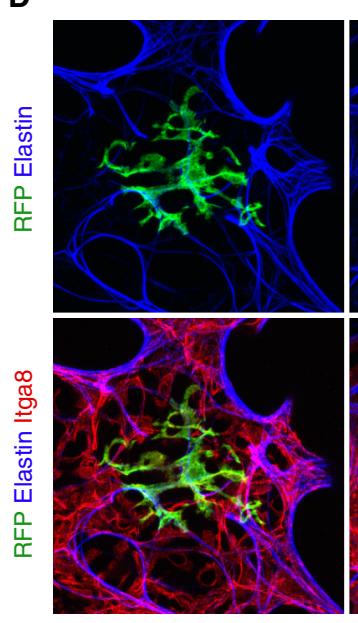

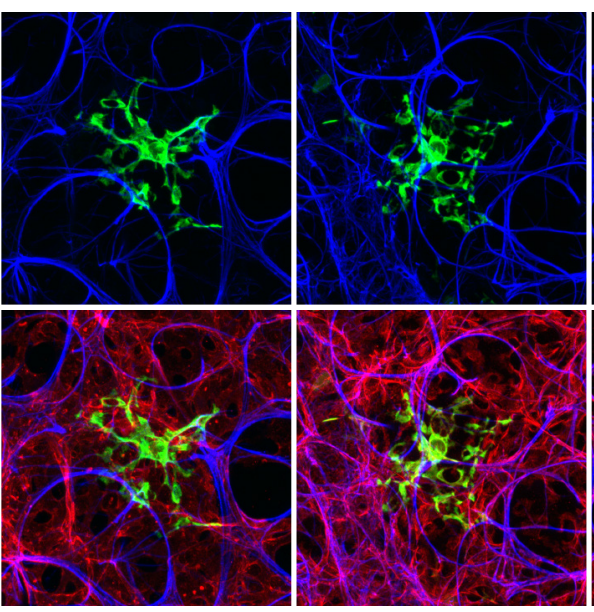

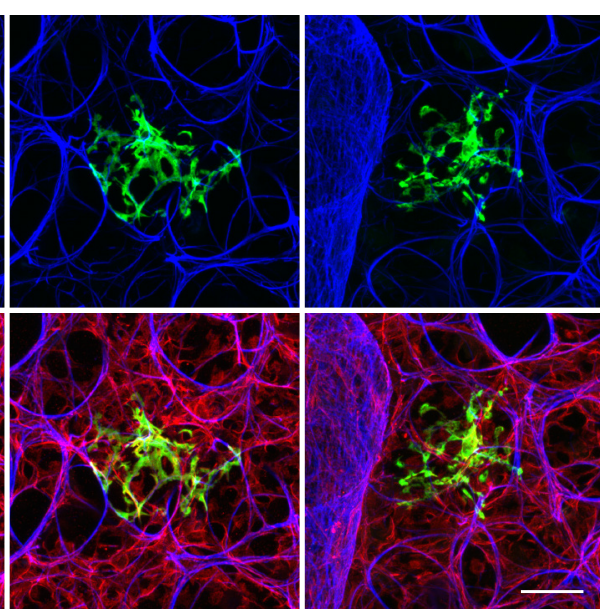




\section{Left lobe}

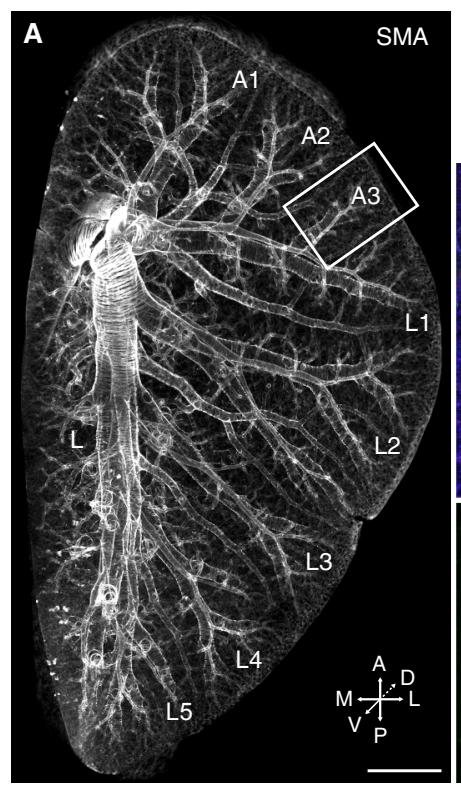

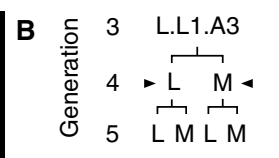
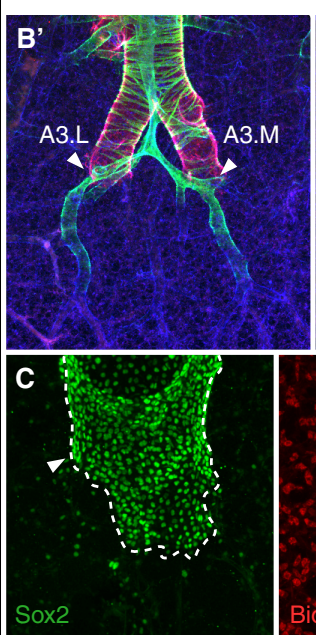

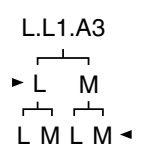
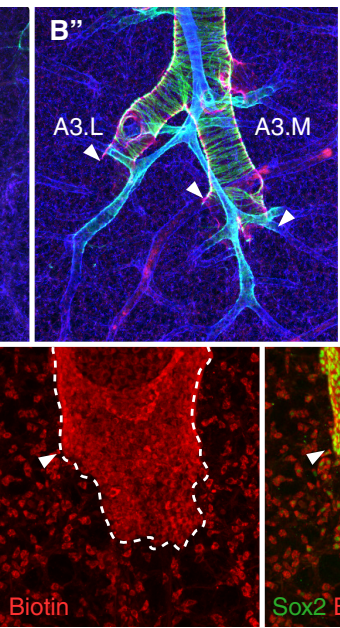

L.L1.A3

L'M.

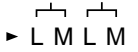

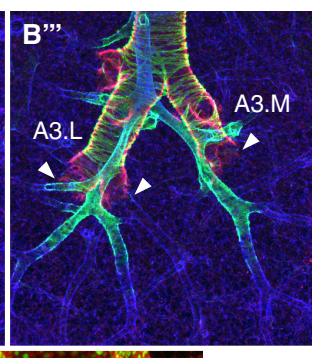

D

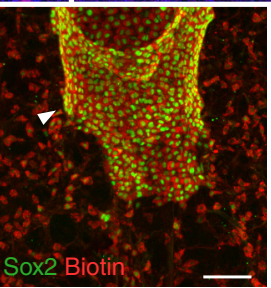

L.L1.A3

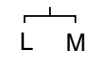

品

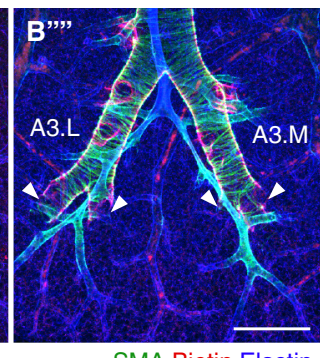

SMA Biotin Elastin \begin{tabular}{llllll} 
& B' & B' & B'” & B'” \\
\hline E15.5 & 42 & 0 & 29 & 29 & $n=14$ \\
E16.5 & 20 & 7 & 20 & 53 & $n=15$ \\
E18.5 & 22 & 11 & 22 & 45 & $n=18$ \\
P60 & 22 & 10 & 20 & 48 & $n=40$ \\
& \% of lungs
\end{tabular} 
bioRxiv preprint doi: https://doi.org/10.1101/2021.12.25.474174; this version posted December 26, 2021. The copyright holder for this preprint (which was not certified by peer review) is the author/funder. All rights reserved. No reuse allowed without permission.

Gillich et al., Figure S8

E17.5 respiratory airway

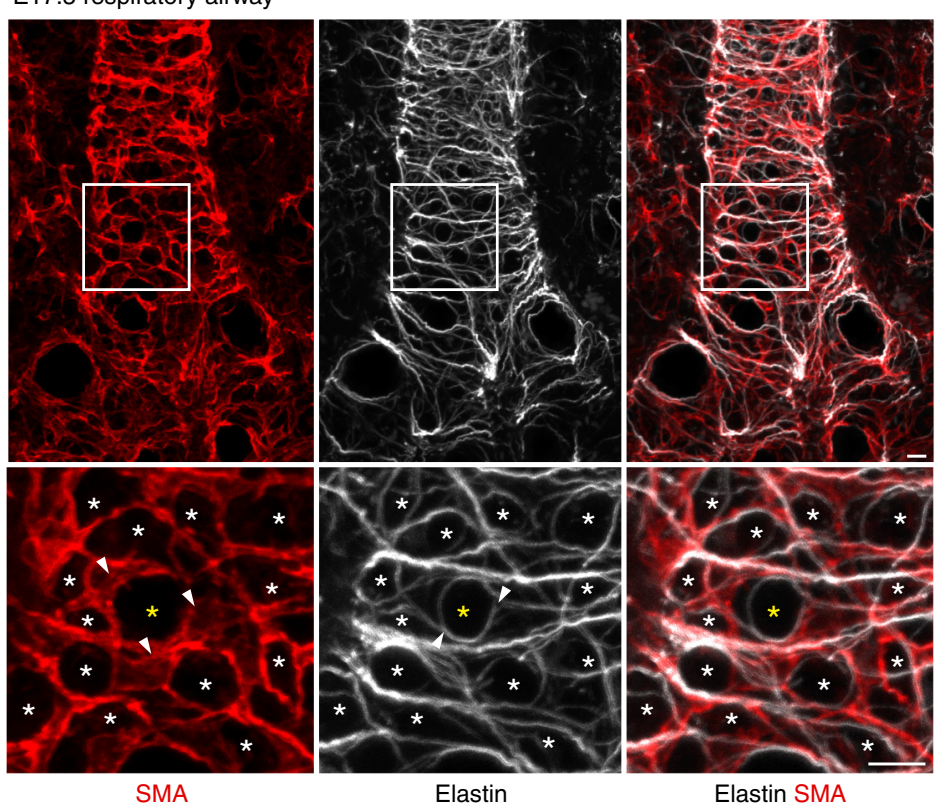


bioRxiv preprint doi: https://doi.org/10.1101/2021.12.25.474174; this version posted December 26, 2021. The copyright holder for this preprint (which was not certified by peer review) is the author/funder. All rights reserved. No reuse allowed without permission.

Gillich et al., Figure S9

\section{A}

Labeling of airway smooth muscle

(Lgr6-CreER; Rosa26-tdTomato)
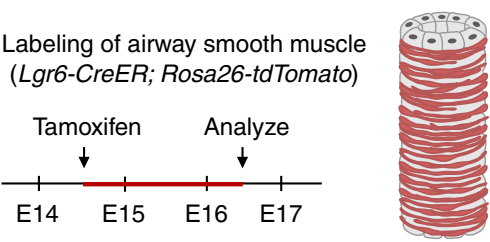

Lgr6-CreER; Rosa26-tdTomato E14.5 > E16.5

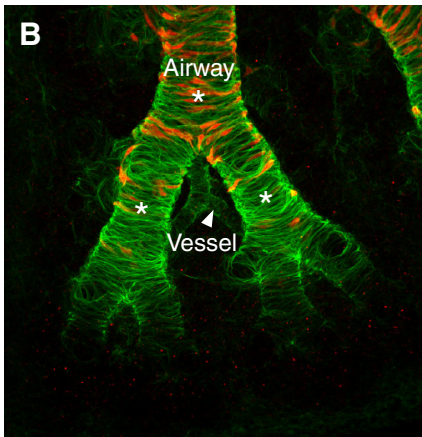

SMA tdTomato

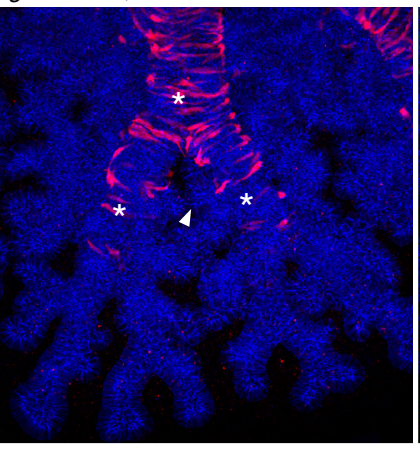

E-cadherin tdTomato

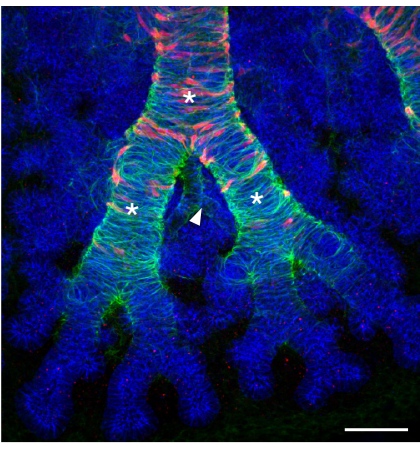

SMA E-cadherin tdTomato 
A Lgr6-CreER; Rosa26-tdTomato

\begin{tabular}{cccccc} 
Tamoxifen \\
E17 & $\mathrm{E} 18$ & P0 & P8 & P9 & P10 \\
\hline & Analyze
\end{tabular}
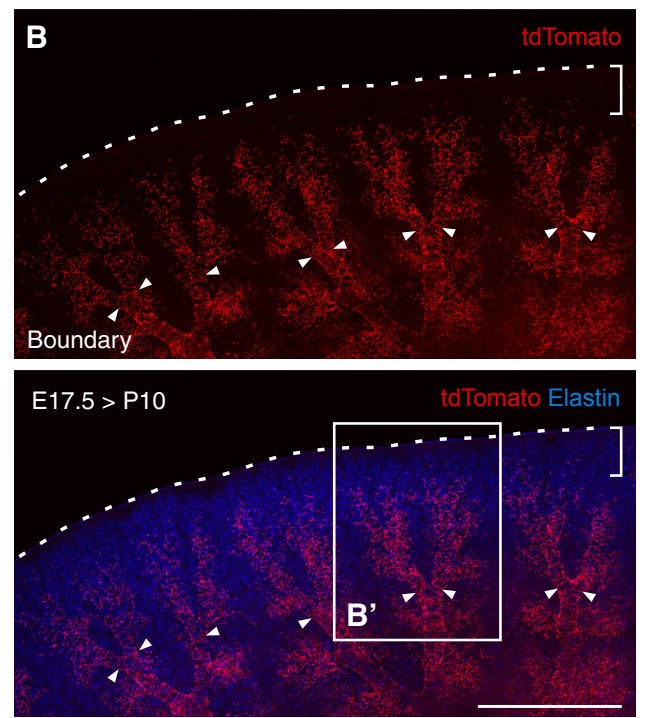

\section{D"}

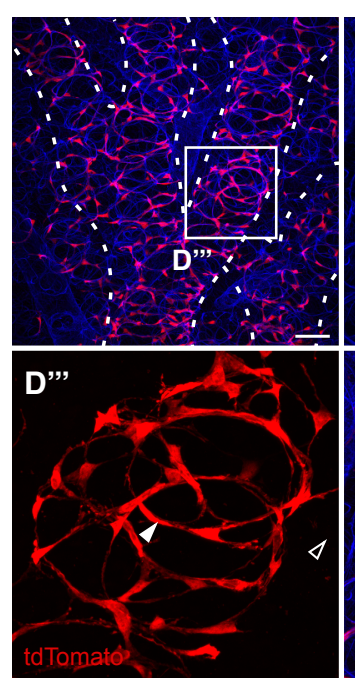

D"'

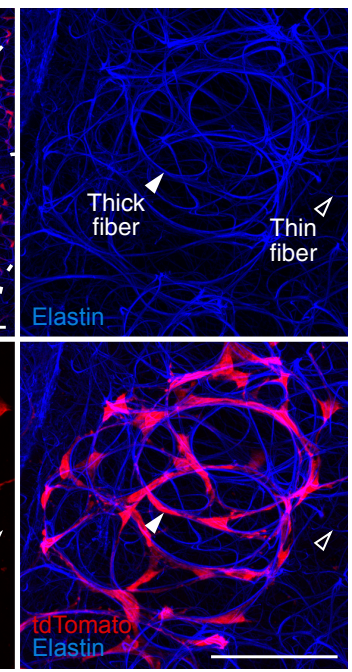

C Lgr6-CreER; Rosa26-tdTomato
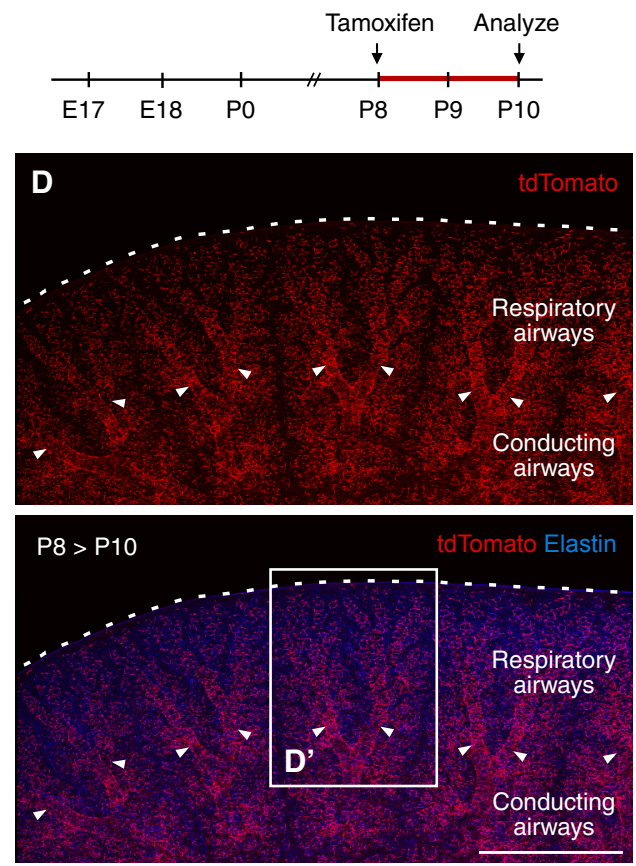

$\mathbf{E}$

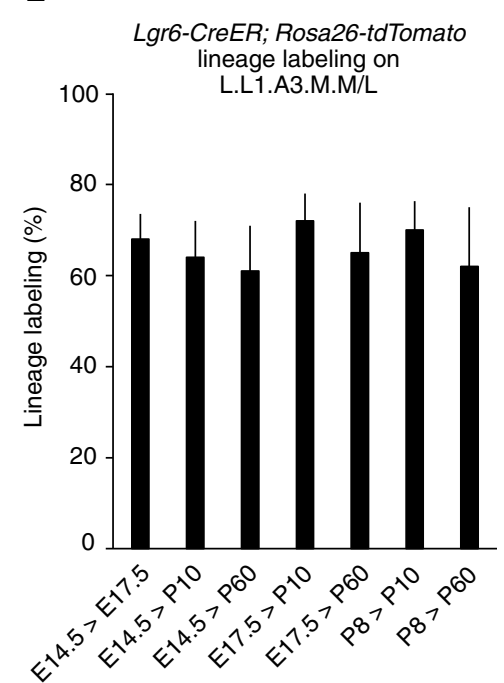

F
B' Lgr6-CreER; Rosa26-tdTomato (E17.5 > P10)

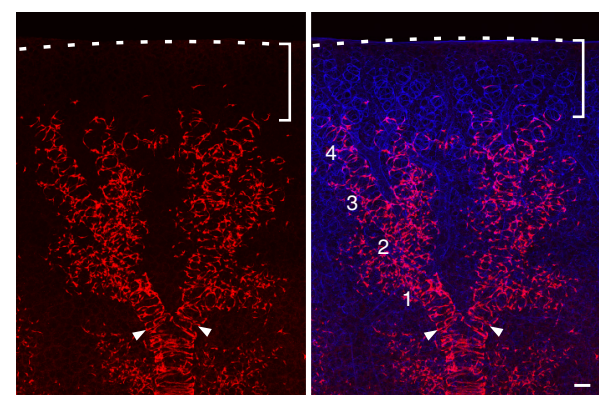

D' Lgr6-CreER; Rosa26-tdTomato (P8 > P10)
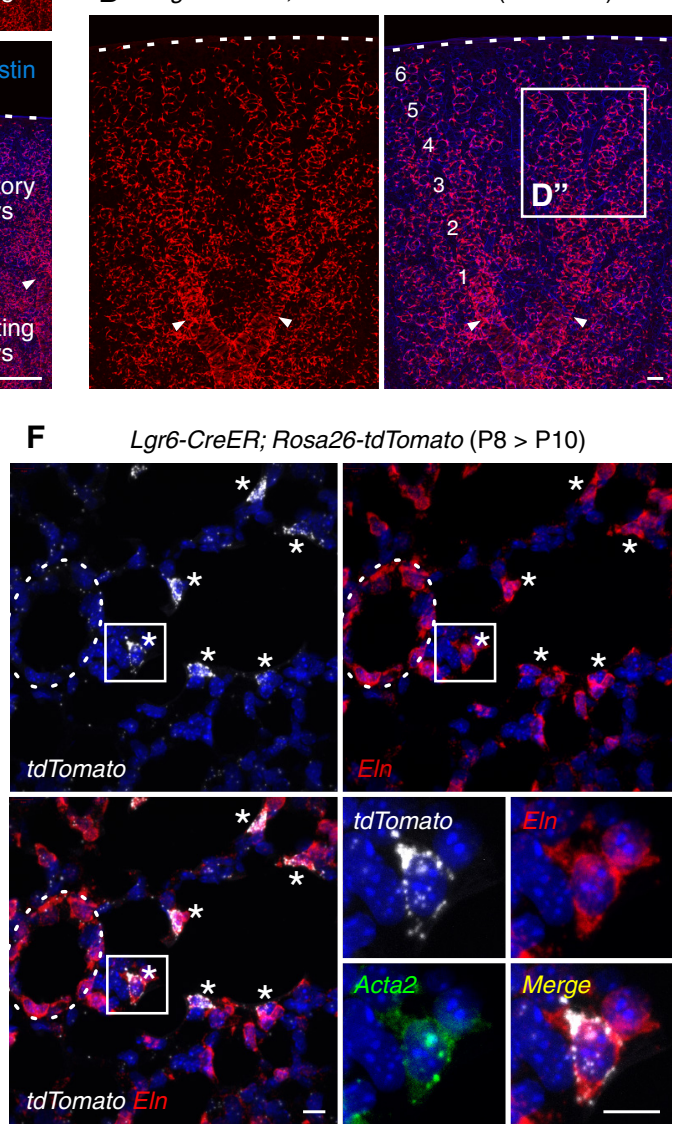

G

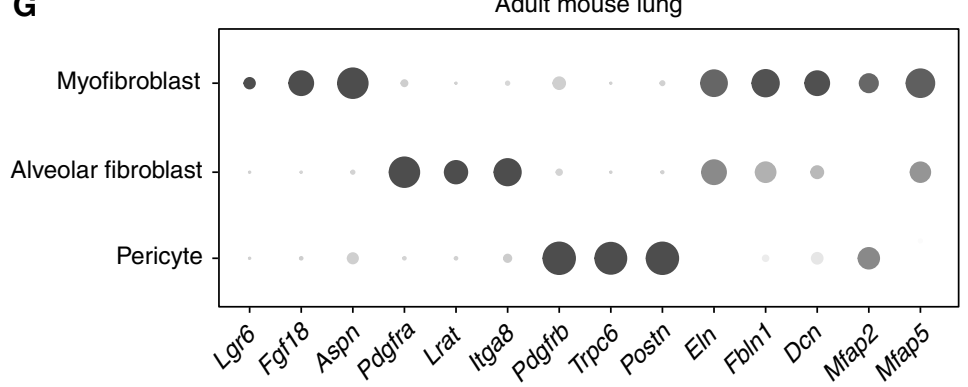

H

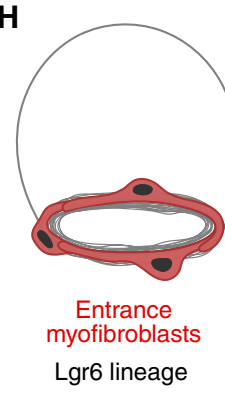

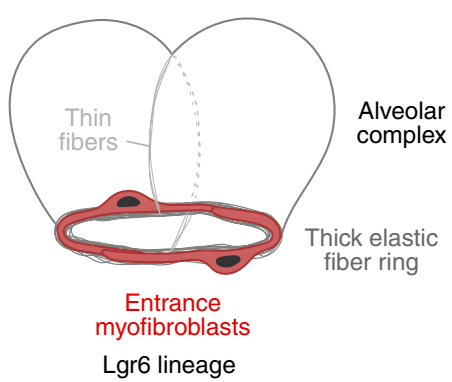


Coherent airway smooth muscle clones

Clone 9

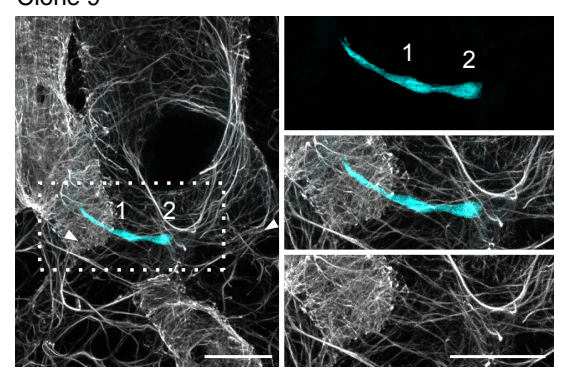

Clone 14

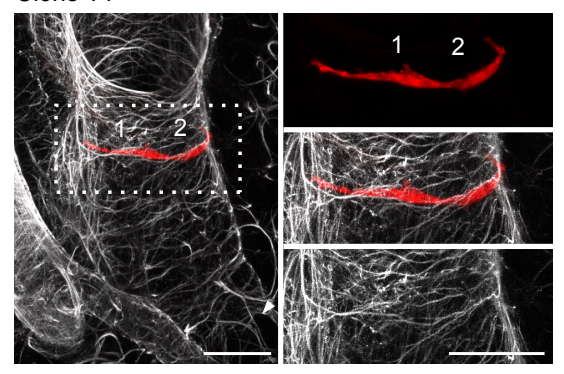

Clone 15

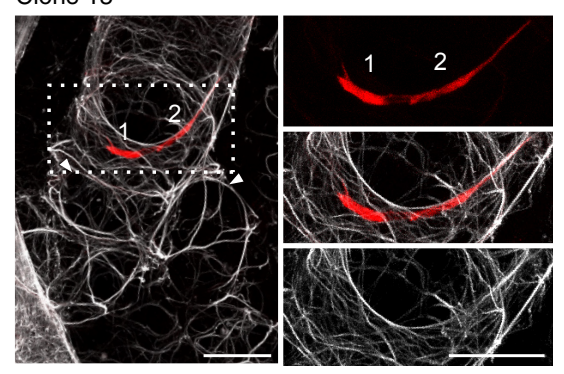

Clone 20

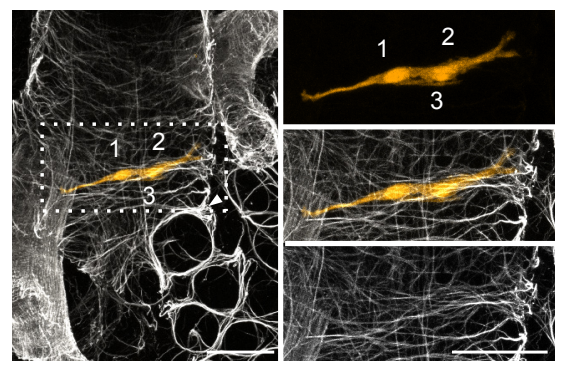

Dispersed airway smooth muscle clones

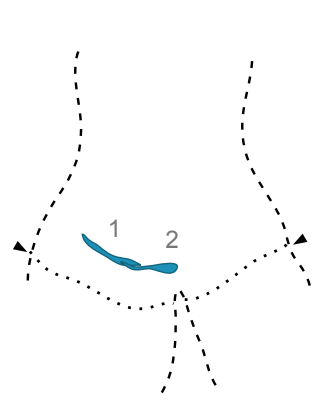

Clone 18
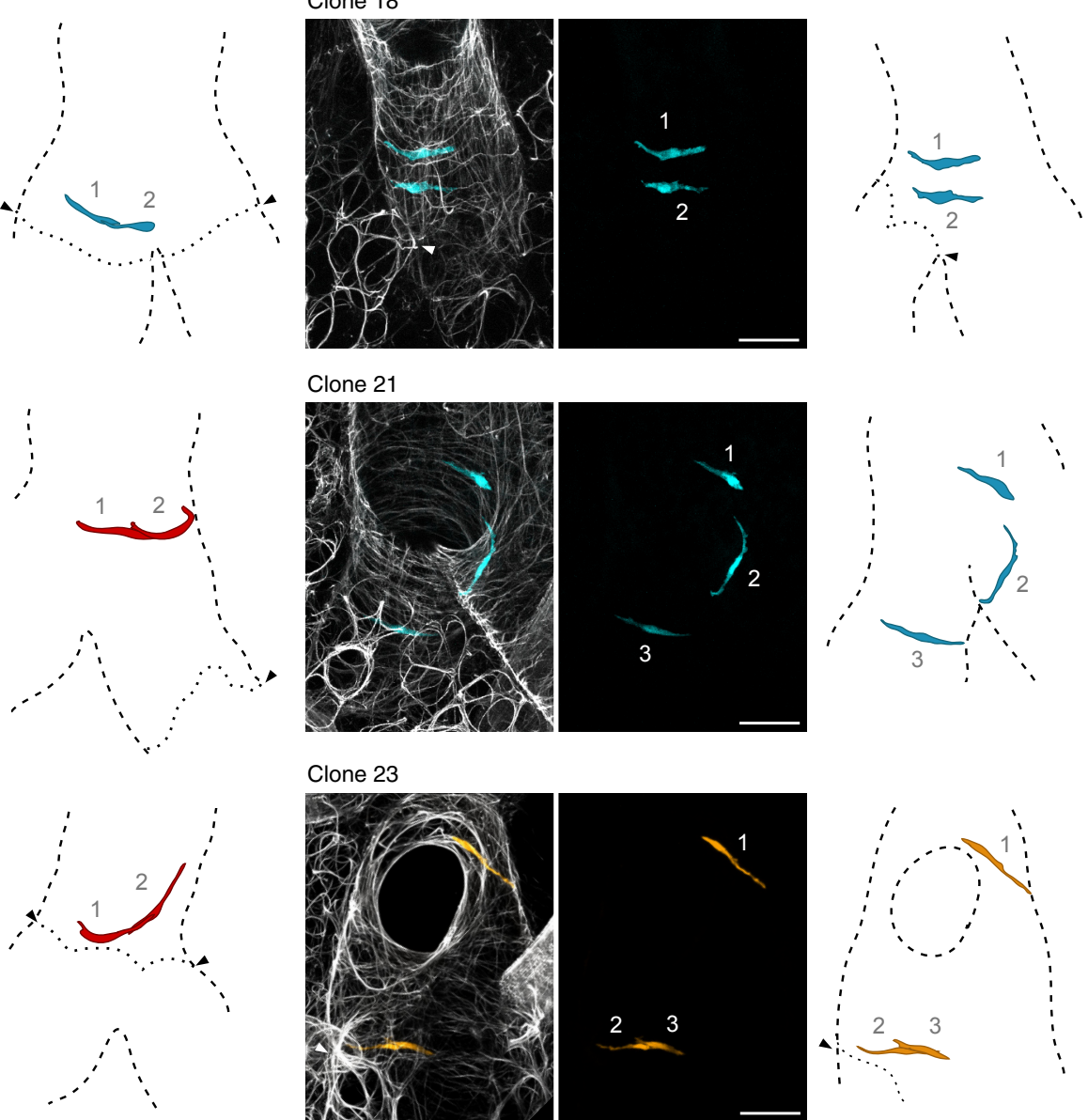

Clone 23
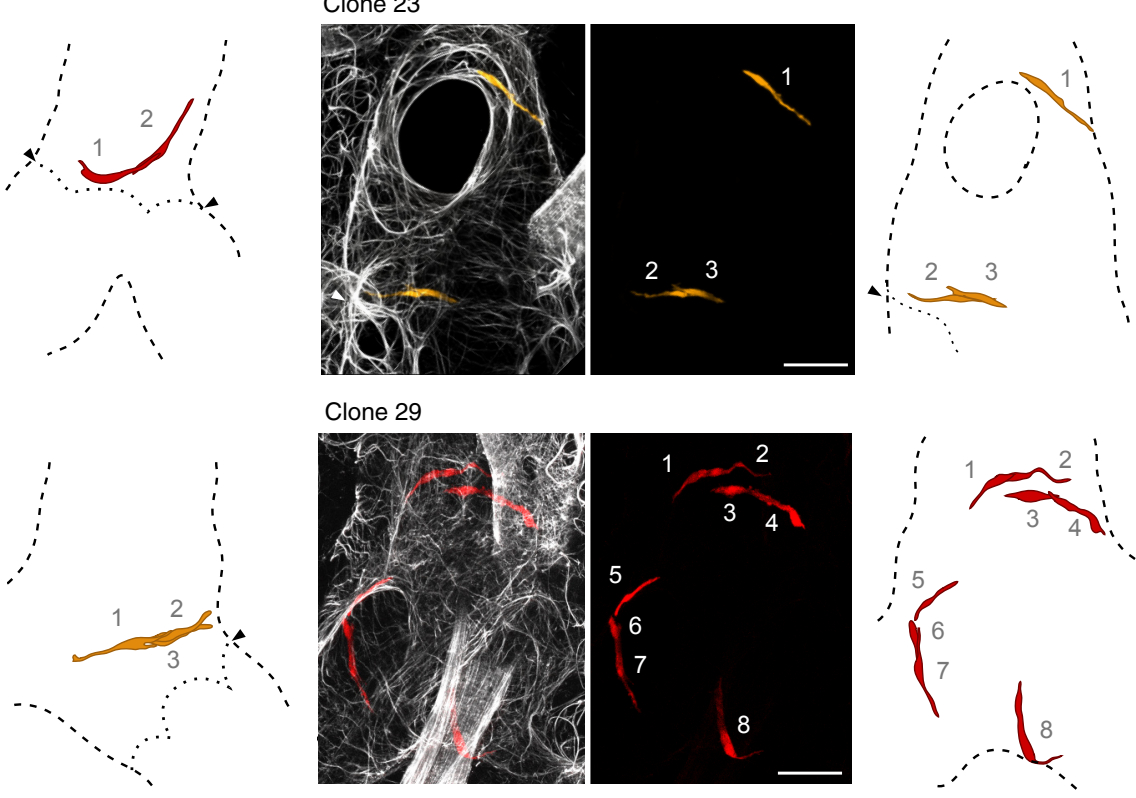

Clone 29
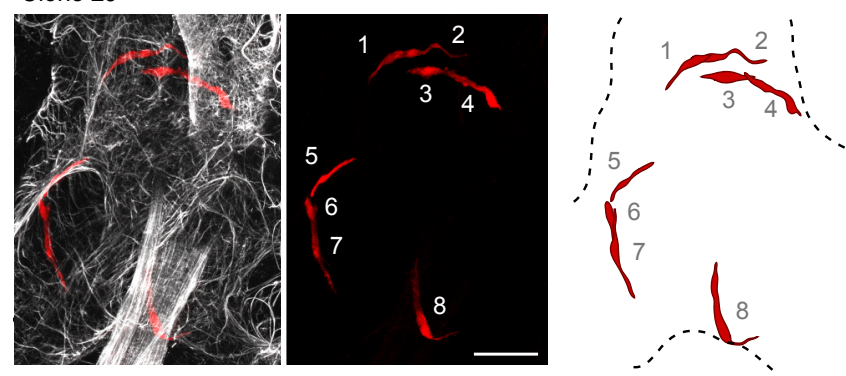

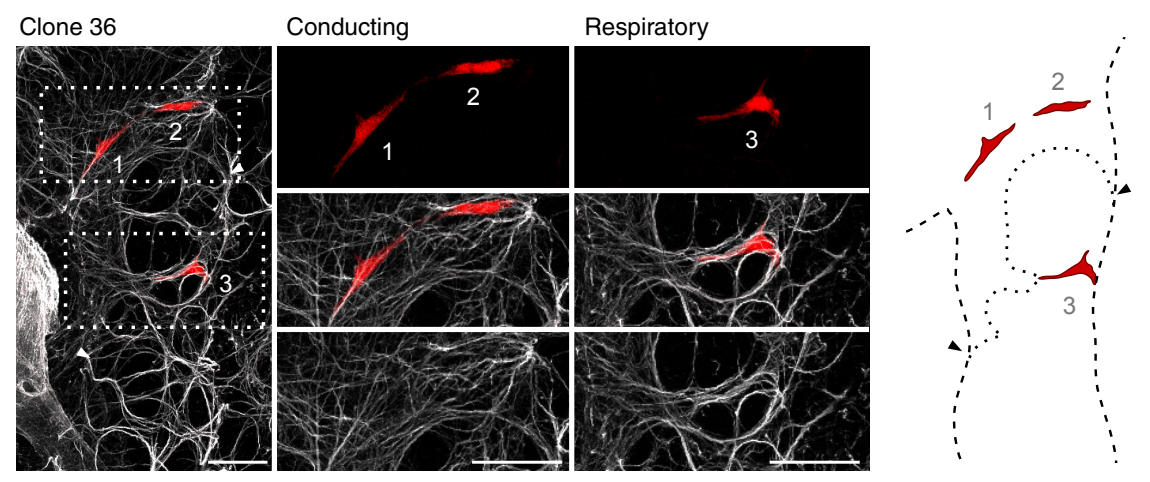

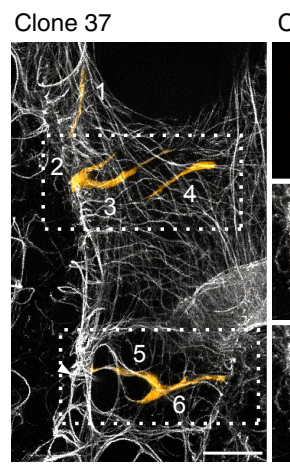

Conducting

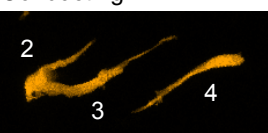

Boundary clones

Clone 31

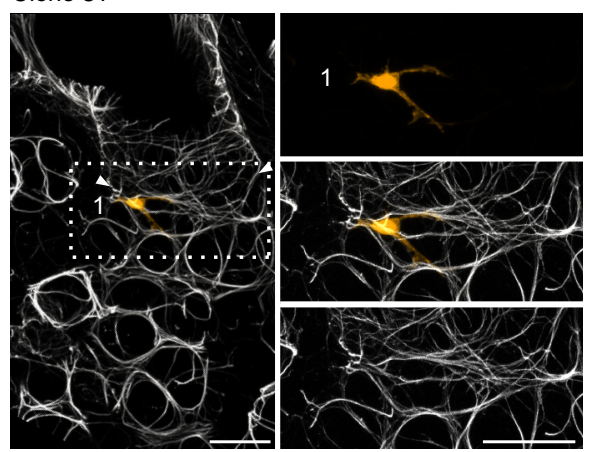

Clone 32

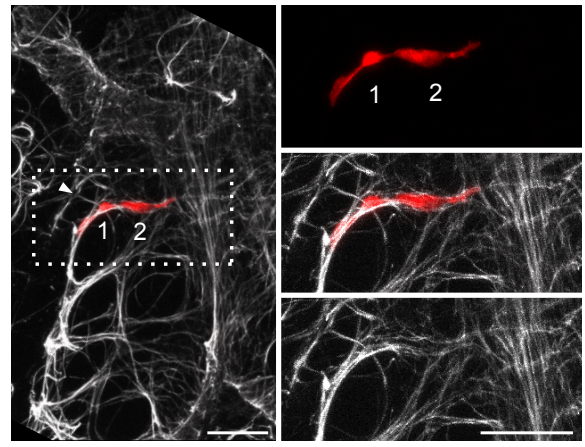

Respiratory
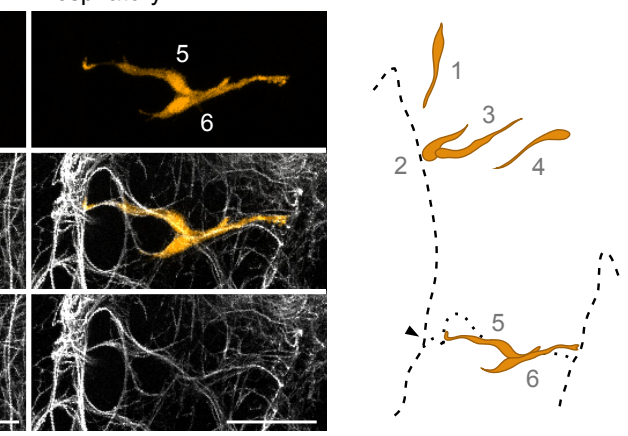
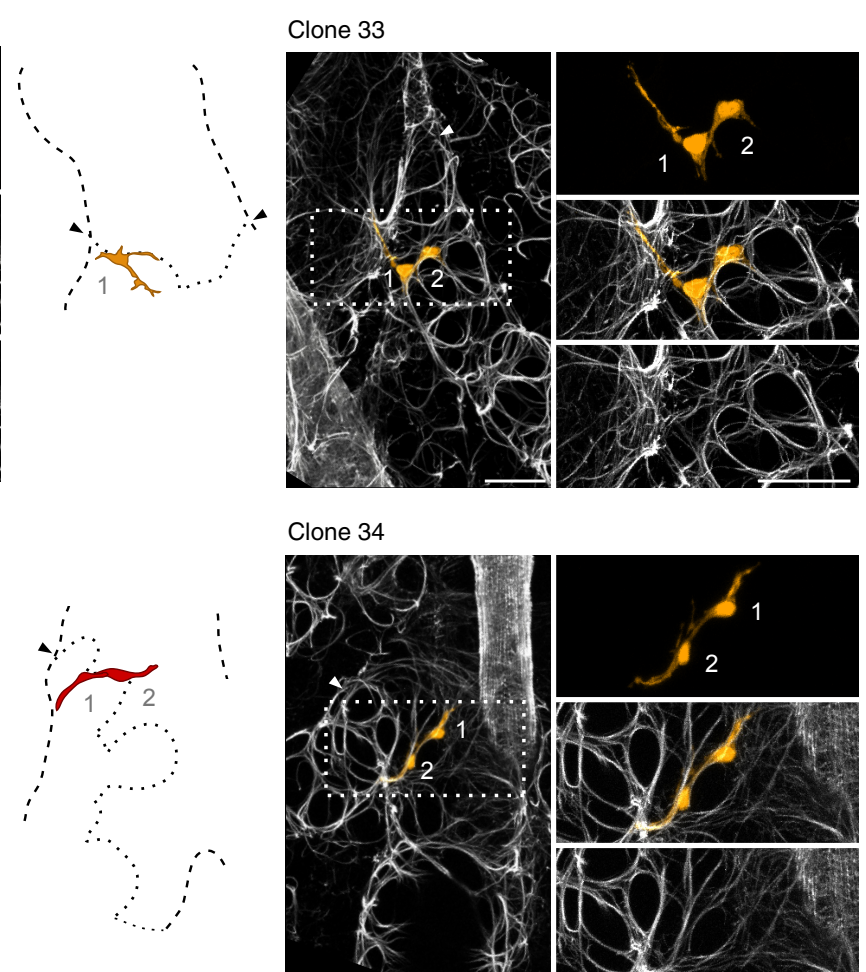

Clone 34

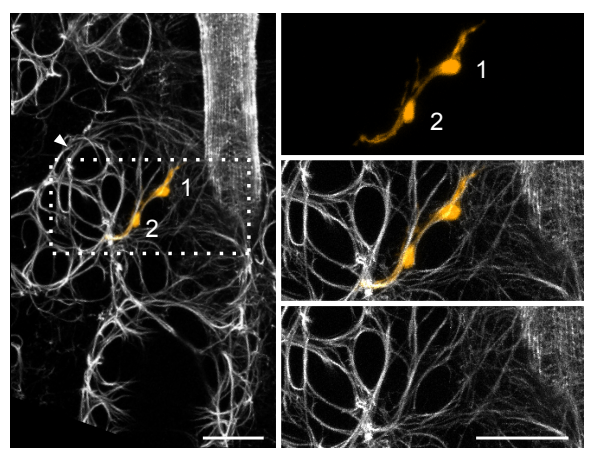

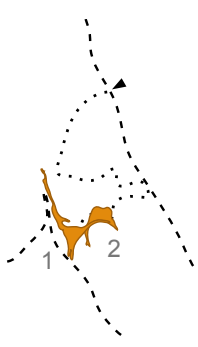

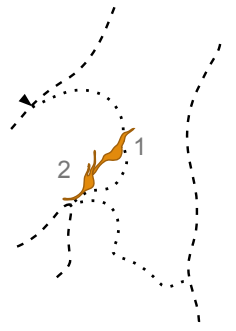


Myofibroblast clones

Clone 48
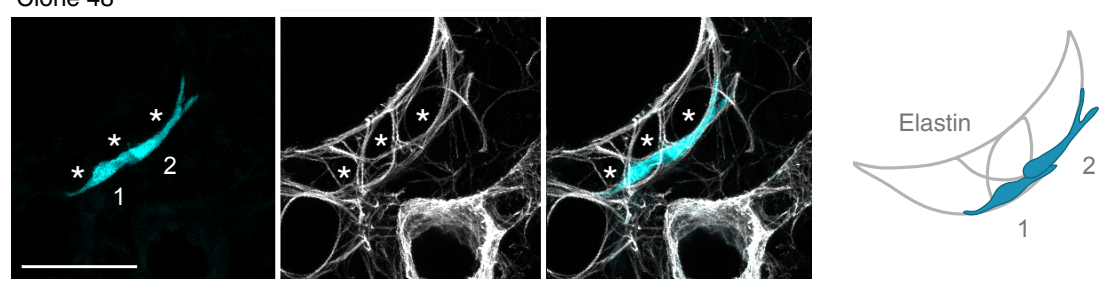

Clone 49
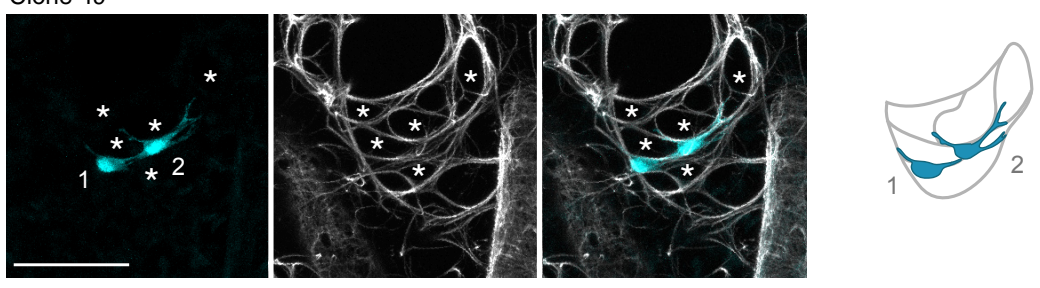

Clone 63
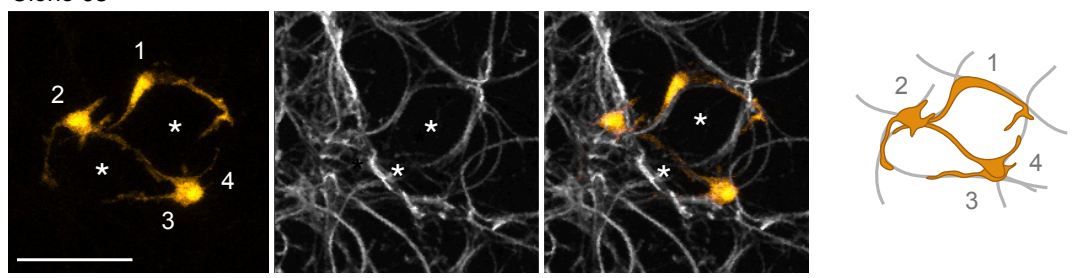

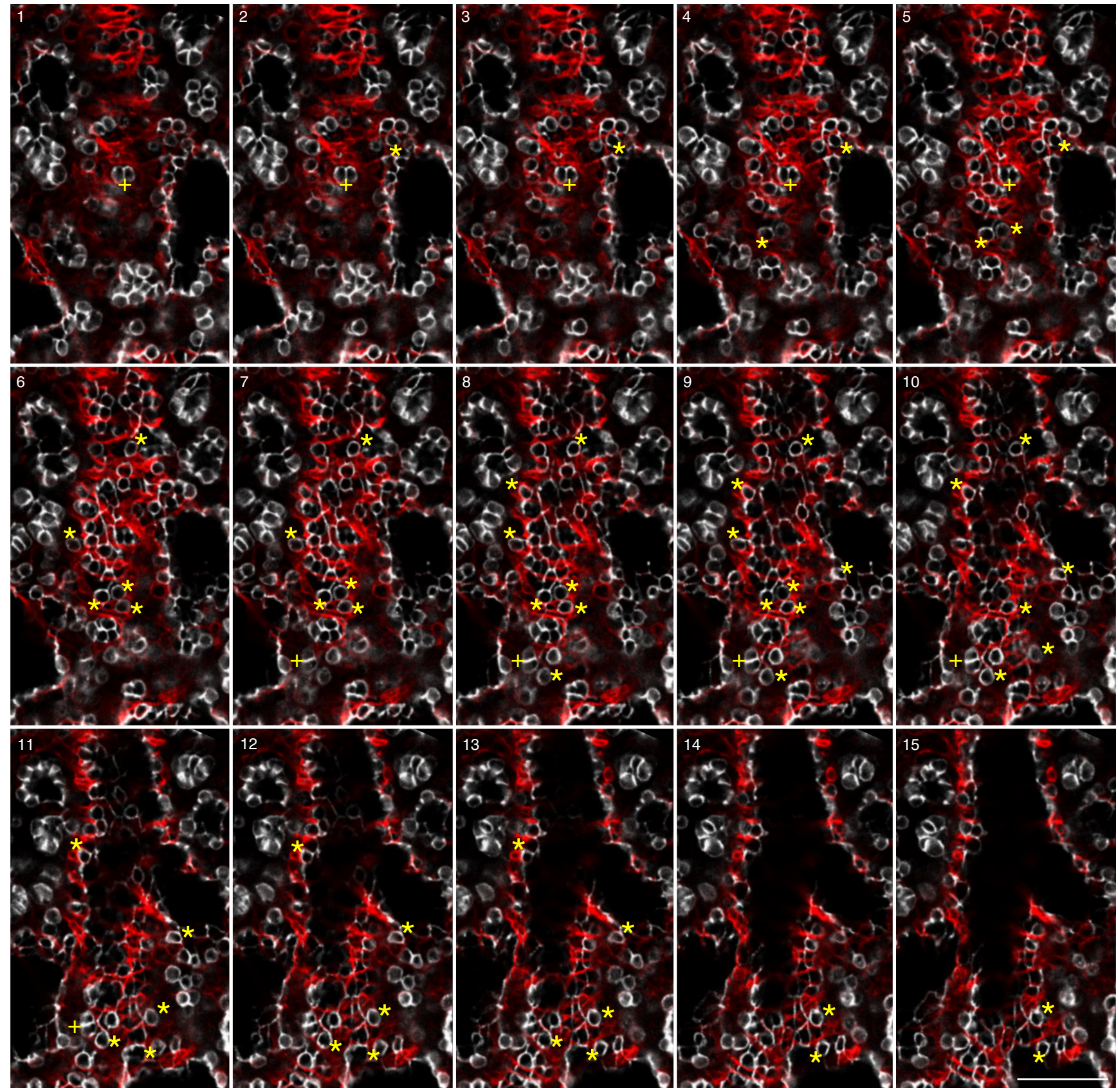

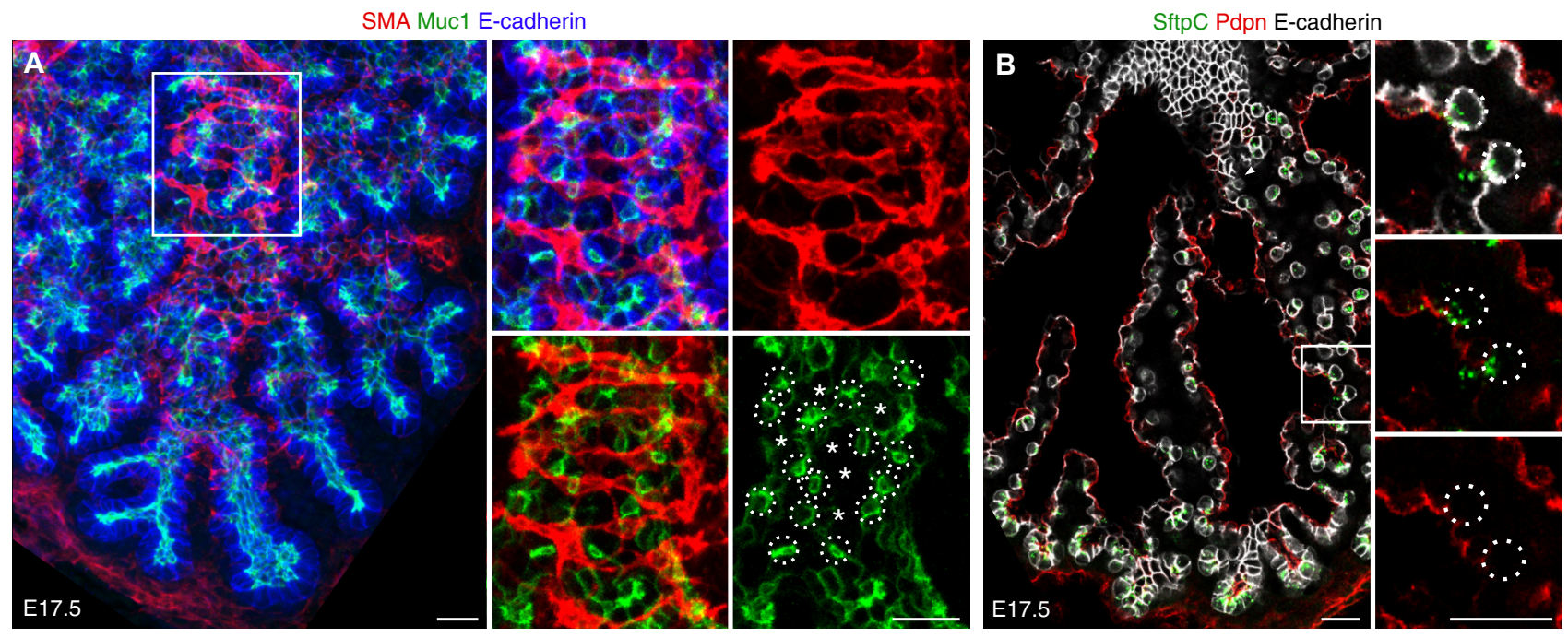

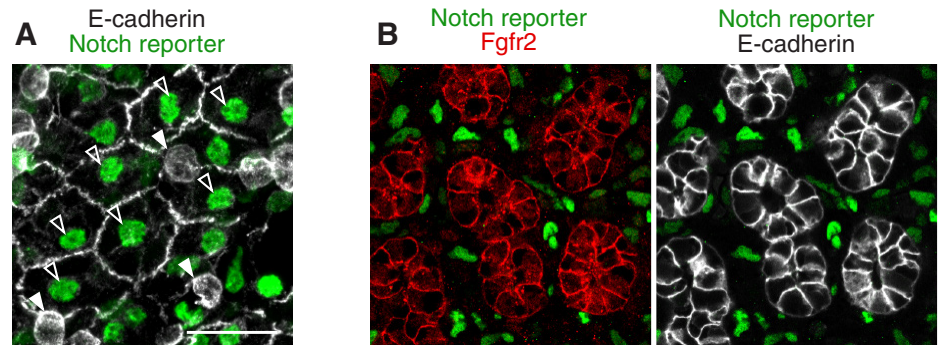

Notch reporter E-cadherin Pecam1

C

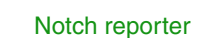

Hes1

Notch reporter Hes1
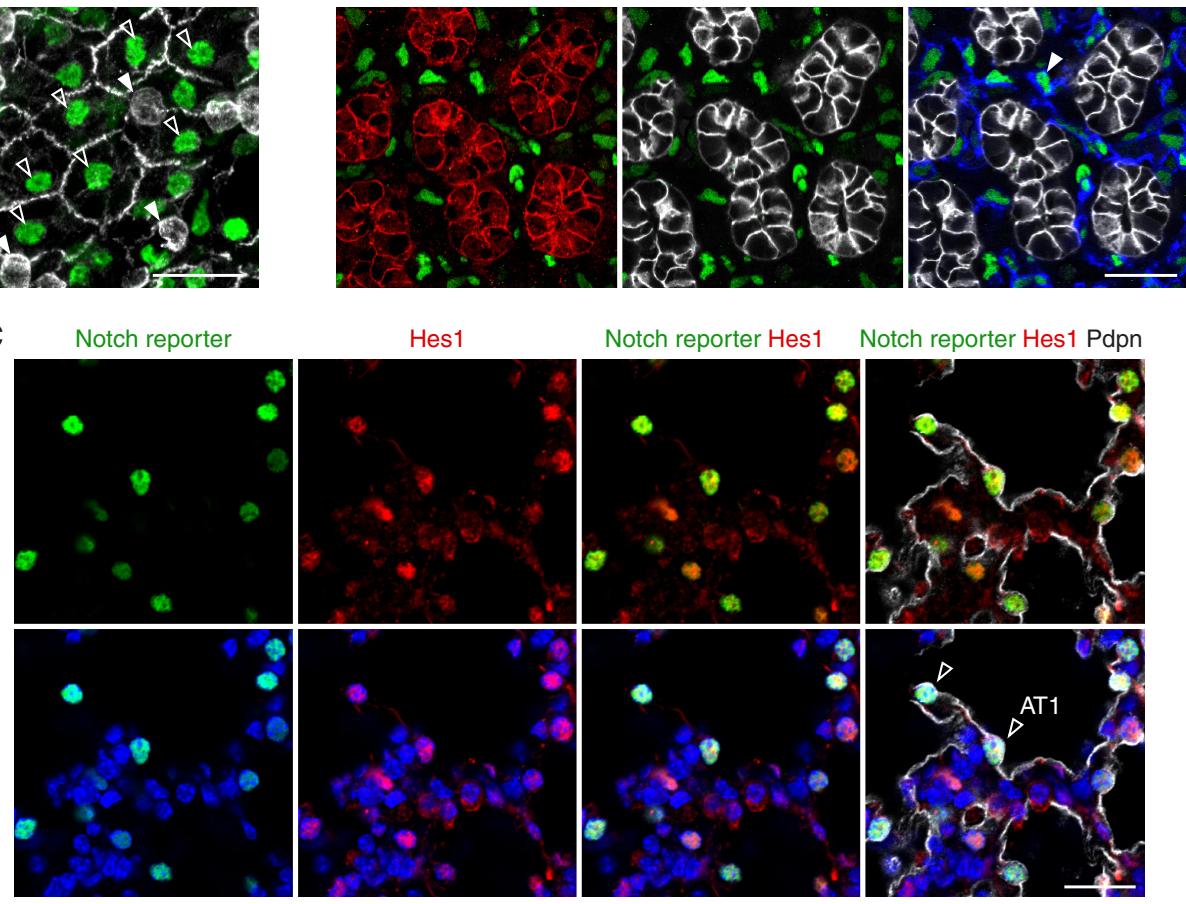

D

Hes1 RAGE

Hes1 RAGE Pdpn

Hes1 RAGE Pdpn DAPI

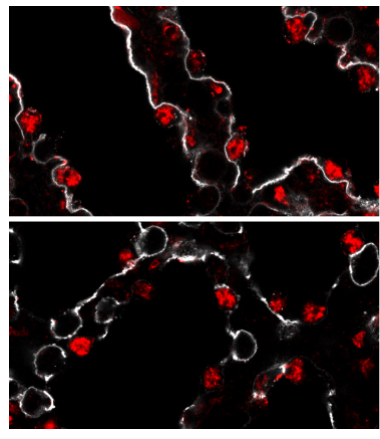

Hes1 E-cadherin

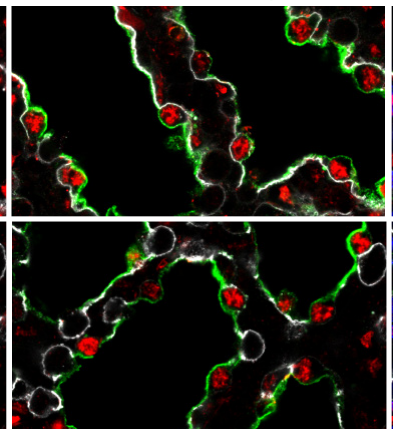

Hes1 E-cadherin Pdpn

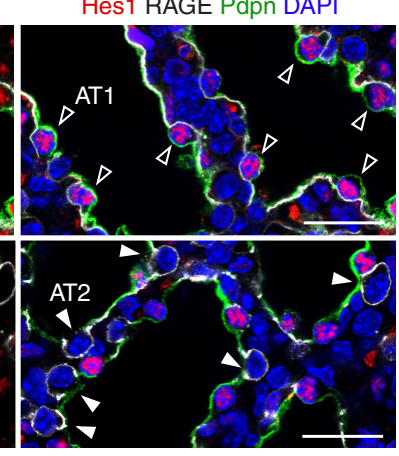

Hes1 E-cadherin Pdpn DAPI

E

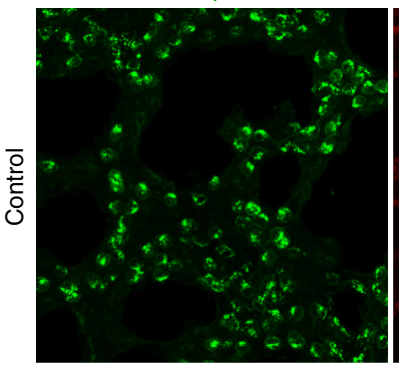

ํํํ

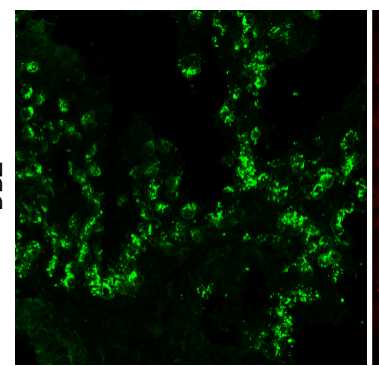

Ki67
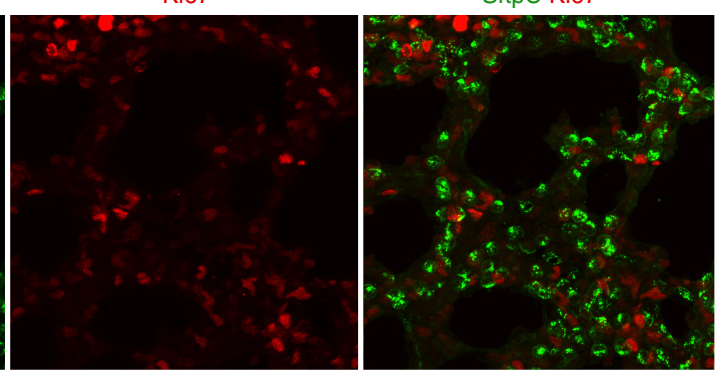

SftpC Ki67 DAPI
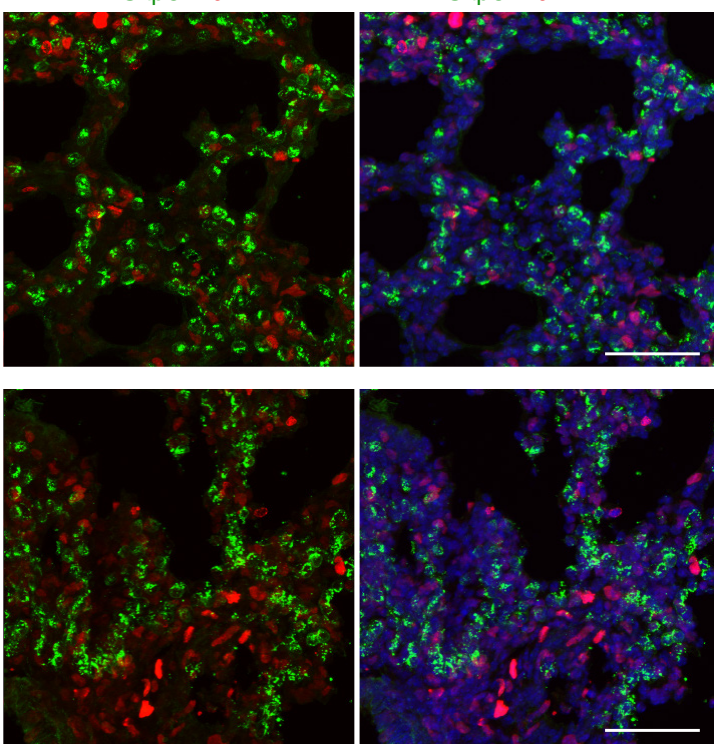
bioRxiv preprint doi: https://doi.org/10.1101/2021.12.25.474174; this version posted December 26, 2021. The copyright holder for this preprint (which was not certified by peer review) is the author/funder. All rights reserved. No reuse allowed without permission.
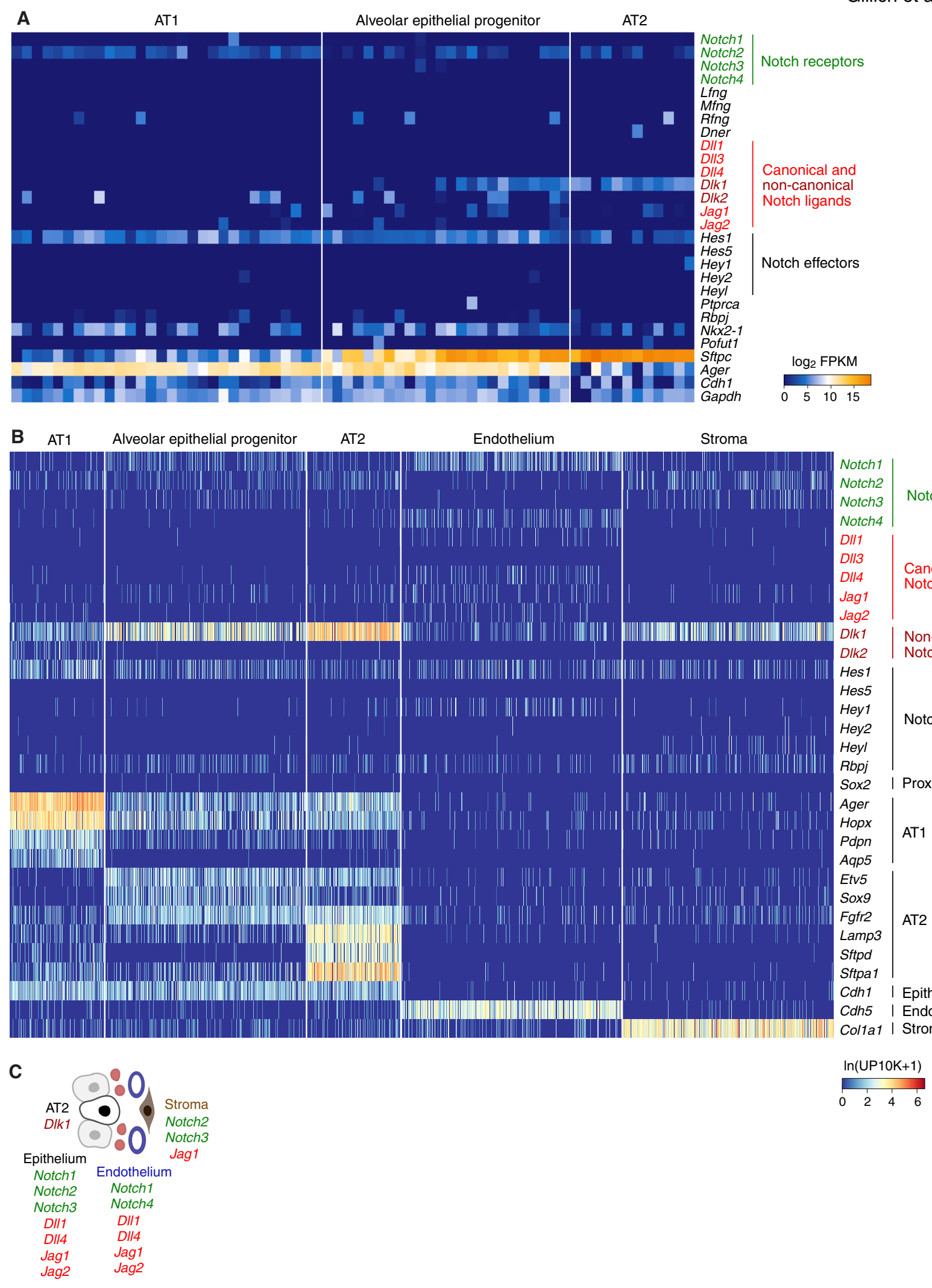
bioRxiv preprint doi: https://doi.org/10.1101/2021.12.25.474174; this version posted December 26,2021 . The copyright holder for this preprint (which was not certified by peer review) is the author/funder. All rights reserved. No reuse allowed without permission.

Table S1. Cre and CreER drivers used for clonal labeling and lineage tracing

\begin{tabular}{|c|c|c|c|c|c|}
\hline Cre/CreER driver & Design & $\begin{array}{l}\text { Recombination } \\
\text { (embryonic) }\end{array}$ & $\begin{array}{l}\text { Recombination } \\
\text { (E17.5-P10) }\end{array}$ & $\begin{array}{l}\text { Recombination } \\
\text { (adult) }\end{array}$ & Reference \\
\hline Shh-GFP-Cre & knock-in & Epithelial cells & Epithelial cells including AT $1 / 2$ cells & Epithelial cells including AT $1 / 2$ cells & $(82)$ \\
\hline Foxa2-CreER & knock-in & Epithelial cells & Epithelial cells including AT $1 / 2$ cells & Epithelial cells including AT $1 / 2$ cells & (81) \\
\hline Hopx-CreER & knock-in & n.d. & n.d. & AT1 cells, aerocytes & $(80)$ \\
\hline VE-cadherin-CreER & transgene & Endothelial cells & Endothelial cells & Endothelial cells & (83) \\
\hline Apelin-CreER & knock-in & Plexus & Plexus, emerging aerocytes & Aerocytes & $(84)$ \\
\hline Aplnr-CreER & transgene & Plexus & Plexus, emerging general capillary cells & General capillary cells & (85) \\
\hline Notch3-CreER & knock-in & n.d. & n.d. & VSM and alveolar pericytes & (88) \\
\hline Pdgfra-CreER & transgene & n.d. & n.d. & Stromal cells including alveolar pericytes & $(86)$ \\
\hline Pdgfra-CreER & knock-in & n.d. & n.d. & Stromal cells including alveolar fibroblasts & ts $(87)$ \\
\hline Gli1-CreER & knock-in & n.d. & n.d. & Stromal cells including alveolar fibroblasts & ts $(89)$ \\
\hline Lgr6-EGFP-IRES-CreERT2 & knock-in & ASM & ASM, entrance myofibroblasts & ASM, entrance myofibroblasts & $(60)$ \\
\hline SMA-CreER & transgene & ASM, VSM & ASM, VSM, myofibroblasts & ASM, VSM & $(90)$ \\
\hline SMMHC-CreER & transgene & ASM, VSM & ASM, VSM, myofibroblasts & ASM, VSM & (64) \\
\hline
\end{tabular}

ASM, airway smooth muscle; VSM, vascular smooth muscle 


\begin{tabular}{|c|c|c|c|c|c|}
\hline Clone No. & No. cells & Position & Cell Type & Arrangement & Color \\
\hline 1 & 1 & Conducting & Airway smooth muscle & $\mathrm{n} / \mathrm{a}$ & Cer \\
\hline 2 & 1 & Conducting & Airway smooth muscle & $n / a$ & Cer \\
\hline 3 & 1 & Conducting & Airway smooth muscle & $\mathrm{n} / \mathrm{a}$ & Cer \\
\hline 4 & 1 & Conducting & Airway smooth muscle & $\mathrm{n} / \mathrm{a}$ & Cer \\
\hline 5 & 1 & Conducting & Airway smooth muscle & $\mathrm{n} / \mathrm{a}$ & mOr \\
\hline 6 & 1 & Conducting & Airway smooth muscle & $\mathrm{n} / \mathrm{a}$ & mOr \\
\hline 7 & 1 & Conducting & Airway smooth muscle & $\mathrm{n} / \mathrm{a}$ & mOr \\
\hline 8 & 1 & Conducting & Airway smooth muscle & $\mathrm{n} / \mathrm{a}$ & $\mathrm{mCh}$ \\
\hline 9 & 2 & Conducting & Airway smooth muscle & Coherent & Cer \\
\hline 10 & 2 & Conducting & Airway smooth muscle & Coherent & Cer \\
\hline 11 & 2 & Conducting & Airway smooth muscle & Coherent & mOr \\
\hline 12 & 2 & Conducting & Airway smooth muscle & Coherent & mOr \\
\hline 13 & 2 & Conducting & Airway smooth muscle & Coherent & mOr \\
\hline 14 & 2 & Conducting & Airway smooth muscle & Coherent & $\mathrm{mCh}$ \\
\hline 15 & 2 & Conducting & Airway smooth muscle & Coherent & $\mathrm{mCh}$ \\
\hline 16 & 2 & Conducting & Airway smooth muscle & Coherent & $\mathrm{mCh}$ \\
\hline 17 & 2 & Conducting & Airway smooth muscle & Coherent & $\mathrm{mCh}$ \\
\hline 18 & 2 & Conducting & Airway smooth muscle & Dispersed & Cer \\
\hline 19 & 2 & Conducting & Airway smooth muscle & Dispersed & Cer \\
\hline 20 & 3 & Conducting & Airway smooth muscle & Coherent & mOr \\
\hline 21 & 3 & Conducting & Airway smooth muscle & Dispersed & Cer \\
\hline 22 & 3 & Conducting & Airway smooth muscle & Dispersed & Cer \\
\hline 23 & 3 & Conducting & Airway smooth muscle & Dispersed & mOr \\
\hline 24 & 4 & Conducting & Airway smooth muscle & Coherent & $\mathrm{mCh}$ \\
\hline 25 & 4 & Conducting & Airway smooth muscle & Dispersed & $\mathrm{mOr}$ \\
\hline 26 & 4 & Conducting & Airway smooth muscle & Dispersed & mOr \\
\hline 27 & 4 & Conducting & Airway smooth muscle & Dispersed & $\mathrm{mCh}$ \\
\hline 28 & 5 & Conducting & Airway smooth muscle & Dispersed & Cer \\
\hline 29 & 8 & Conducting & Airway smooth muscle & Dispersed & $\mathrm{mCh}$ \\
\hline 30 & 13 & Conducting & Airway smooth muscle & Dispersed & $\mathrm{mCh}$ \\
\hline 31 & 1 & Boundary & Hybrid & $n / a$ & $\mathrm{mOr}$ \\
\hline 32 & 2 & Boundary & Hybrid & Coherent & $\mathrm{mCh}$ \\
\hline 33 & 2 & Boundary & Hybrid & Coherent & $\mathrm{mOr}$ \\
\hline 34 & 2 & Boundary & Hybrid & Coherent & $\mathrm{mOr}$ \\
\hline 35 & 2 & Conducting+respiratory & Mixed & Coherent & $\mathrm{mCh}$ \\
\hline 36 & 3 & Conducting+respiratory & Mixed & Dispersed & $\mathrm{mCh}$ \\
\hline 37 & 6 & Conducting+respiratory & Mixed & Dispersed & mOr \\
\hline 38 & 10 & Conducting+respiratory & Mixed & Dispersed & Cer \\
\hline 39 & 1 & Respiratory & Myofibroblast & $\mathrm{n} / \mathrm{a}$ & Cer \\
\hline 40 & 1 & Respiratory & Myofibroblast & $n / a$ & Cer \\
\hline 41 & 1 & Respiratory & Myofibroblast & $n / a$ & Cer \\
\hline 42 & 1 & Respiratory & Myofibroblast & $\mathrm{n} / \mathrm{a}$ & Cer \\
\hline 43 & 1 & Respiratory & Myofibroblast & $n / a$ & $\mathrm{mOr}$ \\
\hline 44 & 1 & Respiratory & Myofibroblast & $n / a$ & $\mathrm{mOr}$ \\
\hline 45 & 1 & Respiratory & Myofibroblast & $n / a$ & $\mathrm{mOr}$ \\
\hline 46 & 1 & Respiratory & Myofibroblast & $n / a$ & $\mathrm{mCh}$ \\
\hline 47 & 1 & Respiratory & Myofibroblast & $\mathrm{n} / \mathrm{a}$ & $\mathrm{mCh}$ \\
\hline 48 & 2 & Respiratory & Myofibroblast & Coherent & Cer \\
\hline 49 & 2 & Respiratory & Myofibroblast & Coherent & Cer \\
\hline 50 & 2 & Respiratory & Myofibroblast & Coherent & Cer \\
\hline 51 & 2 & Respiratory & Myofibroblast & Coherent & Cer \\
\hline 52 & 2 & Respiratory & Myofibroblast & Coherent & $\mathrm{mOr}$ \\
\hline 53 & 2 & Respiratory & Myofibroblast & Coherent & $\mathrm{mOr}$ \\
\hline 54 & 2 & Respiratory & Myofibroblast & Coherent & mOr \\
\hline 55 & 2 & Respiratory & Myofibroblast & Coherent & mOr \\
\hline 56 & 2 & Respiratory & Myofibroblast & Coherent & $\mathrm{mOr}$ \\
\hline 57 & 2 & Respiratory & Myofibroblast & Coherent & $\mathrm{mOr}$ \\
\hline 58 & 2 & Respiratory & Myofibroblast & Coherent & $\mathrm{mCh}$ \\
\hline 59 & 2 & Respiratory & Myofibroblast & Coherent & $\mathrm{mCh}$ \\
\hline 60 & 2 & Respiratory & Myofibroblast & Coherent & $\mathrm{mCh}$ \\
\hline 61 & 3 & Respiratory & Myofibroblast & Coherent & $\mathrm{mOr}$ \\
\hline 62 & 3 & Respiratory & Myofibroblast & Dispersed & $\mathrm{mOr}$ \\
\hline 63 & 4 & Respiratory & Myofibroblast & Coherent & $\mathrm{mOr}$ \\
\hline 64 & 4 & Respiratory & Myofibroblast & Dispersed & $\mathrm{mCh}$ \\
\hline 65 & 8 & Respiratory & Myofibroblast & Dispersed & $\mathrm{mCh}$ \\
\hline 66 & 12 & Respiratory & Myofibroblast & Dispersed & $\mathrm{mOr}$ \\
\hline 67 & 14 & Respiratory & Myofibroblast & Dispersed & $\mathrm{mCh}$ \\
\hline 68 & 17 & Respiratory & Myofibroblast & Dispersed & $\mathrm{mCh}$ \\
\hline
\end{tabular}

